\title{
APPLICATIONS OF AUTOMATA AND GRAPHS: LABELING-OPERATORS IN HILBERT SPACE I
}

\author{
ILWOO CHO AND PALLE E. T. JORGENSEN
}

\begin{abstract}
We show that certain representations of graphs by operators on Hilbert space have uses in signal processing and in symbolic dynamics. Our main result is that graphs built on automata have fractal characteristics. We make this precise with the use of Representation Theory and of Spectral Theory of a certain family of Hecke operators. Let $G$ be a directed graph. We begin by building the graph groupoid $\mathbb{G}$ induced by $G$, and representations of $\mathbb{G}$. Our main application is to the groupoids defined from automata. By assigning weights to the edges of a fixed graph $G$, we give conditions for $\mathbb{G}$ to acquire fractal-like properties, and hence we can have fractaloids or $G$-fractals. Our standing assumption on $G$ is that it is locally finite and connected, and our labeling of $G$ is determined by the "out-degrees of vertices". From our labeling, we arrive at a family of Hecke-type operators whose spectrum is computed. As applications, we are able to build representations by operators on Hilbert spaces (including the Hecke operators); and we further show that automata built on a finite alphabet generate fractaloids. Our Hecke-type operators, or labeling operators, come from an amalgamated free probability construction, and we compute the corresponding amalgamated free moments. We show that the free moments are completely determined by certain scalar-valued functions.
\end{abstract}

A number of recent papers have addressed an intriguing interplay between Discrete Potential Theory on the one hand and Harmonic Analysis / Spectral Theory on an associated family of fractals on the other, with the Sierpinski gasket serving as a preferred model.

This is the first of two papers studying representations of graphs by operators in Hilbert space, and their applications. While graph theory is traditionally considered part of discrete mathematics, in this paper we show that applications of tools from automata and operators on Hilbert spaces yield global results for representations of a class of infinite graphs, as well as spin-off applications. We begin with an outline of the use of automata, and more generally, of finite state models (FSMs) in the processing of numbers, or more importantly in sampling and in quantization of digitized information such as speech signals and digital images. In these models, the finite input states of a particular FSM might be frequency-bands (for example a prescribed pair of high-pass and low-pass digital filters), or a choice of subdivision

Date: Mar., 2008.

1991 Mathematics Subject Classification. 05C62, 05C90, 17A50, 18B40, 46K10, 47A67, 47A99, 47B99.

Key words and phrases. Locally Finite Connected Countable Directed Graphs, Canonical Weighted Graphs, Weighting Processes, Graph Groupoids, Labeled Graph Groupoids, Automata, Graph-Groupoid-Automata, Automata-Trees, Fractaloids, Right Graph von Neumann Algebras, Right Graph $W^{*}$-Probability Spaces, Labeling Operators. 
filters; where the subdivision refers to data sets with self-similarity; such as is typically seen in fractals. Hence, these applications make connections to discrete wavelet algorithms as used in signal and image processing, as well as in science and engineering. If the input-options for a particular FSM are chosen from a prescribed system of low-pass and high-pass filters, the resulting discrete model can then be realized by operators on Hilbert spaces. Similarly, images are digitized into matrix shapes used in computer programs for compression of images.

In a general operator theoretic setting, this paper introduces the relevant representations of the generators of graphs and automata. Hence data from FSMs and graphs are represented with the use of Hilbert space geometry. Recall that digital images are typically given by matrices of pixels, and that spectral analysis and matrix operations can be done with operators in Hilbert space. However, the Hilbert spaces needed in a particular application are typically not immediately apparent from the particular engineering problem under discussion. This paper focused on making the link between graphs and automata on the discrete side to Hilbert space operators and representations on the spectral side.

The potential-theoretic part of the subject may be understood as a mathematical idealization of electrical networks on infinite graphs $G$ (See [41], [42] and [43]). We address two issues from Analysis: Find representations (by operators on Hilbert spaces) of the graph systems $G$, and identify a class of operators whose spectral theory captures significant information about $G$. We focus on the graphs themselves, and our motivation derives in part from work by Strichartz and Kigami (See [44], [45] and [46]) and others for restricted classes of fractals. The focus there is the adaptation of a rescaling and energy-renormalization on graphs and an adaptation to fractal models $X$; for example, to Brownian motion on $X$, or to a version of differential operators on spaces of functions on $X$. Here, our focus is on Operator Theory needed for analysis of graphs and automata (finite-state machines: e.g., [52]) such as are used in for example signal processing algorithm, e.g., those based on a discrete multiresolution (e.g., see [50]).

We begin our paper with an outline of three trends: (i) Hilbert space and Spectral Analysis on the graphs, (ii) Analysis on associated fractals arising from automata, and finally (iii) the interplay between (i) and (ii). We futher stress some of the differences between the two.

The main purpose of this paper is to introduce a new algebraic structures having certain fractal property. In [10], [11], [13], [14] and [15], we introduced graph groupoids induced by countable directed graphs. A graph groupoid is a categorial grouopoid having as a base the set of all vertices of the given graph. We know that every groupoid having only one base element is a group. So, if $G$ is a finite directed graph with graph groupoid $\mathbb{G}$, and if the vertex set $V(G) \subset \mathbb{G}$ consists of only one element, then the graph groupoid $\mathbb{G}$ of this one-vertex-n-loop-edge graph $G$, for $n$ $=|E(G)| \in \mathbb{N}$, is a group; futhermore, it is group-isomorphic to the free group $F_{n}$ with $n$-generators (See [10] and [11]). Here, $E(G)$ denotes the edge set of $G$. We show that the automaton $\mathcal{A}_{G}=<X, \mathbb{F}^{+}\left(G^{\wedge}\right), \varphi, \psi>$, having its alphabet $X=\{1$, $\ldots, 2 n\}$, generates a fractal group $\Gamma \stackrel{\text { Group }}{=} F_{n}$. How about the general case where 
the vertex set contains more than one element? This question is the motivation for our paper. Also, if such generalization is determined suitably, then what are the properties of the corresponding Hecke-type operator (with respect to the natural representation of $\mathbb{G})$ ? We will provide the answers of these questions in the paper.

We realize that, by giving weights on the edges of a given graph $G$, which are determined by the out-degrees of vertices, we can get the corresponding automaton $\mathcal{A}_{G}$. By observing the properties of $\mathcal{A}_{G}$, we can determine the groupoidal version of a fractal property, relative to a fractal group. We will say that a graph groupoid $\mathbb{G}$ is a fractaloid, if $\mathcal{A}_{G}$ acts "fully" on a $\left|V\left(G^{\wedge}\right)\right|$-copies of regular growing directed trees. For example, if a directed graph $G$ is a one-flow circulant graph, then the graph groupoid $\mathbb{G}$ is a fractaloid.

Similar to the construction in [10], we can have a right graph von Neumann algebra $\mathbb{M}_{G}=\overline{\mathbb{C}[\beta(\mathbb{G})]}^{w}$, as a $W^{*}$-subalgebra of the operator algebra $B\left(H_{G}\right)$, consisting of all bounded linear operators on $H_{G}$, where $H_{G}$ is a generalized Fock space, called the graph Hilbert space induced by $\mathbb{G}$. We are interested in the case where $\mathbb{G}$ is a fractaloid. Then, similar to the classical case, we can define the Hecke-type operator $\tau \in \mathbb{M}_{G}$. Instead of considering the pure operator-theoretical data of $\tau$, we will observe the amalgamated free distributional data of $\tau$. Since $\tau$ is self-adjoint, the amalgamated free moments of it contains the operator-valued spectral measure theoretical information. This means that the free moments of $\tau$ will contain the operator-theoretical data of $\tau$. Consequently, in Section 5, we can see that the free moments of $\tau$ is totally depending on the scalar-values, recursively, whenever $\mathbb{G}$ is a fractaloid.

From the theory of algebras of operators on Hilbert space, we will need von Neumann algebra constructions (e.g., [59]), free probability, in particular, amalgamated free products (e.g., [5] and [21]), groupoids and groupoid actions (e.g., [17] and [58]), and Hecke operators (e.g., [25]). In technical discussions, we will use "von Neumann algebras" and " $W^{*}$-algebras" synonynously. If $H$ is a given Hilbert space, the algebra of all bounded operators on $H$ is denoted by $B(H)$. From Graph Theory, we use such notations as "sets of vertices", "sets of edges", "loops" and "degrees", etc. If $G$ is a given directed graph, we introduce a "shadow" construction from a reversal of edges, denoted by $G^{-1}$; see details below. From symbolic dynamics, we shall use fundamentals of "automata", as well as free constructions, such as the free group with multi-generators. Hence, our next section will contain a number of definitions that will needed later.

Since our paper is interdisciplinary and directed at several audiences, we have included details from one area of mathematics which might not be familiar to readers from another.

To get to our main theorems, a number of technical terms must be introduced. We have collected them in Section 2 after Introduction. The essential ones are: graphs, groupoids, and automata. In Section 2.2, we explain the interplay between automata and the graphs, and we introduce "canonical weighted graphs", "shadowed graphs", "fractaloids", and "labeling operators". The first of the results on 
automata are in Section 3.3. From Section 2.3 to Section 2.5, we review facts from Free Probability and von Neumann algebras we will need. Section 5.5 introduces labeling operators, and in Corollary 5.2, we show that these operators are generated by edges of shadowed graphs.

\section{Table of Contents}

1. Introduction 6

2. Preliminaries 9

2.1. Groupoids and Groupoid Actions 9

2.2. Automata and Fractal Groups 11

2.3. Free Probability 15

2.4. Graph Groupoids and Right Graph von Neumann Algebras 18

2.5. $M$-Valued Graph $W^{*}$-Probability Spaces 22

3. Labeled Graph Groupoids and Graph Automata 27

3.1. Labeled Graph Groupoids 28

3.2. The Operation $\theta$ and $\omega_{+} \quad 34$

3.3. Graph Automata 35

4. Fractaloids 38

4.1. Fractaloids $\quad 38$

4.2. Examples $\quad 45$

5. Labeling Operators of Fractaloids 47

5.1. Labeling Operators 47

5.2. Amalgamated Free Distributional Data of Labeling Operators 50

5.3. Labeling Operators of Fractaloids 51

5.4. Refinements of (5.3) 55

5.5. An Example 62

Our main results are Theorems 4.2, 4.4, and Corollary 4.5, 5.5, 5.11, 5.14, 5.15. A number of examples and applications are computed at the end of Section 5 . Theorem 4.2 shows that the pair consisting a weighted graph and a labeled graph groupoid induce a tree (graph), and we display its properties. Futher, we give a necessary and sufficient condition for this to be a fractaloid. In Theorem 4.4 and Corollary 4.5, we show that the automata actions coming from fractaloids act fully on a certain tree. Moreover, they are identified in terms of roots and out-degrees. Theorem 5.7 derives the essential properties of the labeling operators. In Section 5.4 , for a fixed graph $G$, we compute the amalgamated free moments of the labeling operator $T_{G}$; see Corollaries 5.9, 5.11 5.14, and Theorem 5.13.

A graph is a set of objects called vertices (or points or nodes) connected by links called edges (or lines). In a directed graph, the two directions are counted as being distinct directed edges (or arcs). A graph is depicted in a diagrammatic form as a set of dots (for vertices), jointed by curves (for edges). Similarly, a directed graph is depicted in a diagrammatic form as a set of dots jointed by arrowed curves, where the arrows point the direction of the directed edges. 
Throughout this paper, every graph is a locally finite countably directed graph. Recall that we say that a countably directed graph $G$ is locally finite if each vertex of $G$ has only finitely many incident edges. Equivalently, the degree of $v$ is finitely determined. Also, recall that the $\operatorname{degree} \operatorname{deg}(v)$ of a vertex $v$ is defined to be the sum of the out-degree $\operatorname{deg}_{\text {out }}(v)$ and the in-degree $\operatorname{deg}_{\text {in }}(v)$, where

$$
\operatorname{deg}_{\text {out }}(v) \stackrel{\text { def }}{=} \mid\{e \in E(G): e \text { has its initial vertex } v\} \mid
$$

and

$$
\operatorname{deg}_{\text {in }}(v) \stackrel{\text { def }}{=} \mid\{e \in E(G): e \text { has its terminal vertex } v\} \mid \text {. }
$$

Let $G$ be the shadowed graph of $G$, in the sense of [10]. Then we can consider the degree of each vertex of $G^{\wedge}$, too, since $G^{\wedge}$ is also a locally finite countable directed graph. Assume that

$$
N=\max \left\{\operatorname{deg}_{\text {out }}(v): v \in V\left(G^{\wedge}\right)=V(G)\right\} .
$$

Then we can define the labeling set $X=\{1,2, \ldots, N\}$. We assign the weights $\{1, \ldots, N\}$ to all elements in the edge set $E\left(G^{\wedge}\right)$ of the shadowed graph $G^{\wedge}$ of $G$. (This weighting provides the weights of all elements in $\mathbb{G}$, which are the sequences contained in $X_{0}^{\infty}$, where $X_{0}=\{0\} \cup X$.) We will call this process placing the weights onto all elements of $\mathbb{G}$, the labeling process. This labeling process lets us construct the automaton $\mathcal{A}_{G}=\left\langle X_{0}, \mathbb{G}, \varphi, \psi\right\rangle$ induced by the graph groupoid $\mathbb{G}$. If the automaton $\mathcal{A}_{G}$ satisfies certain fractal property; we will call the graph groupoid $\mathbb{G}$ a fractaloid. Clearly, the word "fractaloid" hints at "fractal (graph) groupoid".

As in [10] and [11], we will fix a representation $\left(H_{G}, \beta\right)$ of a graph groupoid $\mathbb{G}$, where $H_{G}$ is the graph Hilbert space and $\beta$ is a certain groupoid action of $\mathbb{G}$. Let $X=\left\{x_{1}, x_{2}, \ldots, x_{N}\right\}$ be the labeling set. Then we can define the operator $\tau_{j} \in$ $B\left(H_{G}\right)$ by

$$
\tau_{j}\left(\xi_{w}\right)=\xi_{w} \xi_{e}=\xi_{w e}, \text { for all } \xi_{w} \in \mathcal{B}_{H_{G}}
$$

for each $j \in X$, where $\mathcal{B}_{H_{G}}$ is the Hilbert basis of $H_{G}$, whenever an edge $e$ has its weight $x_{j}$, and $w e \neq \emptyset$ in $\mathbb{G}$. Then we can have the operator $\tau \in B\left(H_{G}\right)$ defined by

$$
\tau=\sum_{j=1}^{N} \tau_{j}
$$

This Hecke-type operator on $H_{G}$ is said to be the labeling operator of $\mathbb{G}$ on $H_{G}$. We will consider the free distributional data of this operator $\tau$ on $H_{G}$. If $\mathbb{G}$ is a fractaloid, then $\tau$ is self-adjoint. So, the free moments of it contain the spectral theoretical properties of $\tau$. In particular, we can show that the amalgamated free moments $\left(E\left(\tau^{n}\right)\right)_{n=1}^{\infty}$ of $\tau$ are determined by the cardinalities $\left(\eta_{n}\right)_{n=1}^{\infty}$ of certain sets $\sum_{n}^{(N)}$, determined recursively for $n \in \mathbb{N}$. i.e., we can get that 


$$
E\left(\tau^{n}\right)=\eta_{n} \cdot 1_{\mathbb{D}_{G}}, \text { for all } n \in \mathbb{N}
$$

We provide the complete computation of $\left(\eta_{n}\right)_{n=1}^{\infty}$.

\section{INTRODUCTION}

Recently, countable directed graphs have been studied in Pure and Applied Mathematics, because not only are they important noncommutative structures but they help us visualize such structures. Futhermore, the visualization has nice matricial expressions, (sometimes, the operator-valued matricial expressions dependent on) adjacency matrices or incidence matrices of the given graph. In particular, the partial isometries in an operator algebra can be expressed and visualized by directed graphs. The main purpose of this paper is to introduce algebraic and operatoralgebraic structures induced by countable directed graphs. For convenience, as we mentioned at the beginning of this paper, we will restrict our interests to the case where the countable directed graphs are locally finite. In [10] and [11], starting with a countable directed graph, we assign certain algebraic elements gotten from the admissibility, and then we assign partial isometries to those elements. From these operators, we generated a von Neumann algebra and we then considered free probabilistic properties of them (See [10]). In conclusion, we found the nice (free) block structures of such von Neumann algebras and this provides the full characterization of the von Neumann algebras (See [11]).

For a given countable directed graph $G$, we can define the shadow $G^{-1}$ of $G$, which is the oppositely directed graph of $G$. Then we can define the shadowed graph $G^{\wedge}=G \cup G^{-1}$ of $G$ as a directed graph with its vertex set $V\left(G^{\wedge}\right)=V(G)=V\left(G^{-1}\right)$ and its edge set $E\left(G^{\wedge}\right)=E(G) \cup E\left(G^{-1}\right)$. Construct the free semigroupoid $\mathbb{F}^{+}\left(G^{\wedge}\right)$ of the shadowed graph $G^{\wedge}$, as a collection of all vertices and finite paths of $G^{\wedge}$ with its binary operation called the admissibility, where the admissibility is nothing but the direction-depending connectedness of elements in $\mathbb{F}^{+}\left(G^{\wedge}\right)$. Notice that all finite paths in $\mathbb{F}^{+}\left(G^{\wedge}\right)$ are the words in $V\left(G^{\wedge}\right) \cup E\left(G^{\wedge}\right)$. By defining the reduction (RR) on $\mathbb{F}^{+}\left(G^{\wedge}\right)$, we can construct the graph groupoid $\mathbb{G}$. i.e., the graph groupoid $\mathbb{G}$ is a set of all "reduced" words in $E\left(G^{\wedge}\right)$, with the inherited admissibility on $\mathbb{F}^{+}\left(G^{\wedge}\right)$. In fact, this graph groupoid $\mathbb{G}$ is a categorial groupoid with its base $V\left(G^{\wedge}\right)$.

Let $G$ be a countable directed graph and let $\mathbb{G}$ be the corresponding graph groupoid of $G$. Then it is a categorial groupoid (See Section 2.1). It is well-known that every groupoid, having only one base element, is a group. So, we can say that a graph groupoid $\mathbb{G}$ containing only one vertex is a group. We can naturally expect that such graph groupoid $\mathbb{G}$ is induced by the one-vertex-multi-loop-edge graph. As we observed in [10] and [11], such a graph groupoid is indeed a group, group-isomorphic to the free group $F_{n}$ with $n$-generators, where $n$ is the cardinality of loop-edges of $G$. Notice that the free group $F_{n}$ acts fully on the $(2 n)$-regular tree 
$\mathcal{T}_{2 n}$, via the automata action of the corresponding automaton $\left\langle X_{2 n}, F_{n}, \varphi, \psi>\right.$, where $X_{n}=\{1, \ldots, 2 n\}$ and $\varphi$ and $\psi$ are suitable maps defined on $X_{2 n} \times F_{n}$. Moreover, the elements of $F_{n}$ acting on $\mathcal{T}_{2 n}$ generate a fractal group. We will generalize such fractal properties to the case where we have arbitrary groupoids.

For an arbitrary fixed von Neumann algebra $M$ in an operator algebra $B(K)$ of all bounded linear operators on a Hilbert space $K$, we define a crossed product algebra $\mathbb{M}_{G}=M \times_{\beta} \mathbb{G}$ of $M$ and $\mathbb{G}$ via a graph-representation $\beta: \mathbb{G} \rightarrow B(K \otimes$ $\left.H_{G}\right)$, where $H_{G}$ is the Hilbert space with its Hilbert basis $\left\{\xi_{w}: w \in F P_{r}\left(G^{\wedge}\right)\right\}$, where

$$
F P_{r}\left(G^{\wedge}\right) \stackrel{\text { def }}{=} \mathbb{G} \backslash\left(V\left(G^{\wedge}\right) \cup\{\emptyset\}\right) .
$$

A graph-representation $\beta$ is a nonunital intertwined partial representation or a groupoid action, determining the bounded operators $\beta_{w}$ on $K \otimes H_{G}$ satisfying that

and

$$
\beta_{w}(m) R_{w} R_{w}^{*}=R_{w}^{*} m R_{w}
$$

$$
\beta_{v}(m)=m
$$

for all $m \in M, w \in F P_{r}\left(G^{\wedge}\right)$ and $v \in V\left(G^{\wedge}\right)$, where $R_{w}$ are the right multiplication operators on $H_{G}$ with their symbols $\xi_{w}$, for all $w \in \mathbb{G}$. The adjoint $R_{w}^{*}$ of $R_{w}$ are defined to be $R_{w^{-1}}$, for all $w \in \mathbb{G}$.

Definition 1.1. The crossed product algebra $\mathbb{M}_{G}=M \times_{\beta} \mathbb{G}$ is the von Neumann algebra generated by $M$ and $\left\{R_{w}: w \in \mathbb{G}\right\}$, satisfying the above $\beta$-relations. We call $\mathbb{M}_{G}$ a right graph von Neumann algebra induced by $G$ over $M$. A right graph von Neumann algebra $\mathbb{M}_{G}$ has its canonical $W^{*}$-subalgebra $\mathbb{D}_{G} \stackrel{\text { def }}{=} \underset{v \in V\left(G^{\wedge}\right)}{\oplus}(M$. $\left.R_{v}\right)$, called the $M$-diagonal subalgebra of $\mathbb{M}_{G}$.

Recall that, in [10], we observed the (left) multiplication operators $L_{w}$ 's, for all $w \in \mathbb{G}$, instead of using right multiplication operators $R_{w}$ 's. Similar to [10], we construct an amalgamated $W^{*}$-probability space $\left(\mathbb{M}_{G}, E\right)$ over $\mathbb{D}_{G}$, where $E: \mathbb{M}_{G}$ $\rightarrow \mathbb{D}_{G}$ is the canonical conditional expectation. Under this setting, we realized that $\mathbb{M}_{G}$ is *-isomorphic to a $\mathbb{D}_{G}$-valued reduced free product $*_{\mathbb{D}_{G}}^{r} \mathbb{M}_{e}$ of the $\mathbb{D}_{G}$-free blocks $\mathbb{M}_{e}$ indexed by directed edges $e \in E(G)$, where

$$
\mathbb{M}_{e} \stackrel{\text { def }}{=} v N\left(M \times_{\beta} \mathbb{G}_{e}, \mathbb{D}_{G}\right) \text {, for all } e \in E(G)
$$

Here, $\mathbb{G}_{e}$ is a substructure (or a subgroupoid) of $\mathbb{G}$, consisting of all reduced words in $\left\{e, e^{-1}\right\}$, with the inherited admissibility on $\mathbb{G}$, for all $e \in E(G)$. Notice that the reduction of the free product " $*_{\mathbb{D}_{G}}^{r}$ " is completely dependent upon the admissibility on the graph groupoid $\mathbb{G}$. 
In [11], we characterized $\mathbb{D}_{G}$-free blocks of graph von Neumann algebras. Similar to [11], we can characterize the $\mathbb{D}_{G}$-free blocks $\mathbb{M}_{e}$ 's of our right graph von Neumann algebra $\mathbb{M}_{G}$. Because of the setting, in fact, the results are almost same. Especially, we show that each $\mathbb{D}_{G}$-free block $\mathbb{M}_{e}$ of $\mathbb{M}_{G}$ is $*$-isomorphic to a certain von Neumann algebra contained in either $\mathcal{M}_{l p}^{\beta}$ or $\mathcal{M}_{n o n-l p}^{\beta}$, where

$$
\mathcal{M}_{l p}^{\beta}=\left\{v N\left(M \times_{\lambda_{e}} \mathbb{Z}, \mathbb{D}_{G}\right): \lambda_{e}=\left.\beta\right|_{\mathbb{S}_{e}}, e \text { is a loop edge }\right\}
$$

and

$$
\mathcal{M}_{\text {non-lp }}^{\beta}=\left\{v N\left(M_{2}^{\beta_{e}}(M), \mathbb{D}_{G}\right): e \text { is a non-loop edge }\right\}
$$

where $M \times_{\lambda^{(e)}} \mathbb{Z}$ is a classical group crossed products of $M$ and the infinite cyclic abelian group $\mathbb{Z}$ via a group action $\lambda^{(e)}$ satisfying that $\lambda_{(e)}=\left.\beta\right|_{\mathbb{G}_{e}}$, and where $M_{2}^{\beta_{e}}(M)$ is a $W^{*}$-subalgebra of $M_{2}(M)=M \otimes_{\mathbb{C}} M_{2}(\mathbb{C})$ satisfying the $\beta_{e^{-}}$ relation: $\beta_{e}(m) R_{e} R_{e}^{*}=R_{e}^{*} m R_{e}$, for all $m \in M$ and for $e \in E(G)$, where $M_{2}(\mathbb{C})$ is the matricial algebra generated by all $(2 \times 2)$-matrices. In particular, if $M=\mathbb{C}$, we can conclude that each $\mathbb{D}_{G}$-free block $\mathbb{M}_{e}$ of $\mathbb{M}_{G}$ is $*$-isomorphic to either $L^{\infty}(\mathbb{T})$ or $M_{2}(\mathbb{C})$, where $\mathbb{T}$ is the unit circle in $\mathbb{C}$, for all $e \in E(G)$. This characterization says that the study of right graph von Neumann algebras is the investigation of graph groupoids and the above two types of von Neumann algebras.

In this paper, we only consider the case where the fixed von Neumann algebra $M$ is $\mathbb{C}$. Then the right graph von Neumann algebras $\mathbb{M}_{G}=\mathbb{C} \times_{\beta} \mathbb{G}$ are all $*$-isomorphic to $M_{G}=\overline{\mathbb{C}}[\mathbb{G}]^{w}$, as $W^{*}$-subalgebras of $B\left(H_{G}\right)$, for all graph-representations $\beta$, by the linearity of $\beta$ on $\mathbb{C}$. We will say that the von Neumann algebra $M_{G}$ is the right graph von Neumann algebra of $G$.

We will consider the labeling operator $\tau$ of $\mathbb{G}$, which is an analogue of classical Hecke-type operators (of groups). As an element of the right graph von Neumann algebra $M_{G}$, we can verify that $\tau$ has its decomposition $\tau=\oplus_{j=1}^{N} \tau_{j}$, where

$$
N \stackrel{\text { def }}{=} \max \left\{\operatorname{deg}_{\text {out }}(v): v \in V\left(G^{\wedge}\right)\right\}
$$

and

$$
\tau_{j}\left(\xi_{w}\right) \stackrel{\text { def }}{=} \begin{cases}\xi_{w} \xi_{e}=\xi_{w e} & \begin{array}{l}
\text { if } w e \neq \emptyset \text { and } \\
e \text { has its weight } j
\end{array} \\
\xi_{w} \xi_{\emptyset}=\xi_{\emptyset}=0 & \begin{array}{l}
\text { if there is no edge } e \text { such that } \\
e \text { has its weight } j \text { and } w e \neq \emptyset .
\end{array}\end{cases}
$$

The study of the labeling operator $\tau$, itself, is interesting in Operator Theory and Quantum Physics, but we will concentrate on observing its free probabilistic data, where $\mathbb{G}$ is a fractaloid. Consequently, we can show that, if a graph groupoid $\mathbb{G}$ of a locally finite directed graph $G$ is a fractaloid, then $\tau$ is self-adjoint and hence the free moments of $\tau$ contain the Spectral Theoretical data of $\tau$. Moreover, we can show that the free moments of $\tau$ are totally depending on certain scalar-values. 


\section{Preliminaries}

In this Section, we introduce the definitions and concepts which we will use in this paper. We will review groupoids, groupoid actions, automata and fractal groups. Also, we introduce and discuss right graph von Neumann algebras.

\subsection{Groupoids and Groupoid Actions.}

While (categorial) groupoids and their actions are used in many areas of mathematics (e.g., [17], [27], [34], and [58]), here we use them in connections with graphs and representations, and we open with the necessary definitions.

We say an algebraic structure $(\mathcal{X}, \mathcal{Y}, s, r)$ is a (categorial) groupoid if it satisfies that (i) $\mathcal{Y} \subset \mathcal{X}$, (ii) for all $x_{1}, x_{2} \in \mathcal{X}$, there exists a partially-defined binary operation $\left(x_{1}, x_{2}\right) \mapsto x_{1} x_{2}$, for all $x_{1}, x_{2} \in \mathcal{X}$, depending on the source map $s$ and the range map $r$ satisfying that:

(ii-1) $x_{1} x_{2}$ is well-determined, whenever $r\left(x_{1}\right)=s\left(x_{2}\right)$ and in this case, $s\left(x_{1}\right.$ $\left.x_{2}\right)=s\left(x_{1}\right)$ and $r\left(x_{1} x_{2}\right)=r\left(x_{2}\right)$, for $x_{1}, x_{2} \in \mathcal{X}$,

(ii-2) $\left(x_{1} x_{2}\right) x_{3}=x_{1}\left(x_{2} x_{3}\right)$, if they are well-determined in the sense of (ii-1), for $x_{1}, x_{2}, x_{3} \in \mathcal{X}$

(ii-3) if $x \in \mathcal{X}$, then there exist $y, y^{\prime} \in \mathcal{Y}$ such that $s(x)=y$ and $r(x)=y^{\prime}$, satisfying $x=y x y^{\prime}$ (Here, the elements $y$ and $y^{\prime}$ are not necessarily distinct),

(ii-4) if $x \in \mathcal{X}$, then there exists a unique element $x^{-1}$ for $x$ satisfying $x x^{-1}=$ $s(x)$ and $x^{-1} x=r(x)$.

Thus, every group is a groupoid $(\mathcal{X}, \mathcal{Y}, s, r)$ with $|\mathcal{Y}|=1$ (and hence $s=r$ on $\mathcal{X}$ ). This subset $\mathcal{Y}$ of $\mathcal{X}$ is said to be the base of $\mathcal{X}$. Remark that we can naturally assume that there exists the empty element $\emptyset$ in a groupoid $\mathcal{X}$. The empty element $\emptyset$ means the products $x_{1} x_{2}$ are not well-defined, for some $x_{1}, x_{2} \in \mathcal{X}$. Notice that if $|\mathcal{Y}|=1$ (equivalently, if $\mathcal{X}$ is a group), then the empty word $\emptyset$ is not contained in the groupoid $\mathcal{X}$. However, in general, whenever $|\mathcal{Y}| \geq 2$, a groupoid $\mathcal{X}$ always contain the empty word. So, if there is no confusion, we will naturally assume that the empty element $\emptyset$ is contained in $\mathcal{X}$.

It is easily checked that our graph groupoid $\mathbb{G}$ of a finite directed graph $G$ is indeed a groupoid with its base $V\left(G^{\wedge}\right)$. i.e., every graph groupoid $\mathbb{G}$ of a countable 
directed graph $G$ is a groupoid $\left(\mathbb{G}, V\left(G^{\wedge}\right), s, r\right)$, where $s(w)=s(v w)=v$ and $r(w)=r\left(w v^{\prime}\right)=v^{\prime}$, for all $w=v w v^{\prime} \in \mathbb{G}$ with $v, v^{\prime} \in V\left(G^{\prime}\right)$. i.e., the vertex set $V\left(G^{\wedge}\right)=V(G)$ is a base of $\mathbb{G}$.

Let $\mathcal{X}_{k}=\left(\mathcal{X}_{k}, \mathcal{Y}_{k}, s_{k}, r_{k}\right)$ be groupoids, for $k=1,2$. We say that a map $f: \mathcal{X}_{1}$ $\rightarrow \mathcal{X}_{2}$ is a groupoid morphism if (i) $f$ is a function, (ii) $f\left(\mathcal{Y}_{1}\right) \subseteq \mathcal{Y}_{2}$, (iii) $s_{2}(f(x))=$ $f\left(s_{1}(x)\right)$ in $\mathcal{X}_{2}$, for all $x \in \mathcal{X}_{1}$, and (iv) $r_{2}(f(x))=f\left(r_{1}(x)\right)$ in $\mathcal{X}_{2}$, for all $x \in \mathcal{X}_{1}$. If a groupoid morphism $f$ is bijective, then we say that $f$ is a groupoid-isomorphism, and the groupoids $\mathcal{X}_{1}$ and $\mathcal{X}_{2}$ are said to be groupoid-isomorphic.

Notice that, if two countable directed graphs $G_{1}$ and $G_{2}$ are graph-isomorphic, via a graph-isomorphism $g: G_{1} \rightarrow G_{2}$, in the sense that (i) $g$ is bijective from $V\left(G_{1}\right)$ onto $V\left(G_{2}\right)$, (ii) $g$ is bijective from $E\left(G_{1}\right)$ onto $E\left(G_{2}\right)$, (iii) $g(e)=g\left(v_{1} e\right.$ $\left.v_{2}\right)=g\left(v_{1}\right) g(e) g\left(v_{2}\right)$ in $E\left(G_{2}\right)$, for all $e=v_{1} e v_{2} \in E\left(G_{1}\right)$, with $v_{1}, v_{2} \in V\left(G_{1}\right)$, then the graph groupoids $\mathbb{G}_{1}$ and $\mathbb{G}_{2}$ are groupoid-isomorphic. More generally, if $G_{1}$ and $G_{2}$ have graph-isomorphic shadowed graphs $\hat{G_{1}}$ and $\hat{G_{2}}$, then $\mathbb{G}_{1}$ and $\mathbb{G}_{2}$ are groupoid-isomorphic.

Proposition 2.1. Let $G_{1}$ and $G_{2}$ be countable directed graph with their graph groupoids $\mathbb{G}_{1}$ and $\mathbb{G}_{2}$, respectively. If the shadowed graphs $\hat{G_{1}}$ and $\hat{G_{2}}$ are graphisomorphic, then $\mathbb{G}_{1}$ and $\mathbb{G}_{2}$ are groupoid-isomorphic.

Proof. Suppose $\hat{G_{1}}$ and $\hat{G_{2}}$ are graph-isomorphic, via a graph-isomorphism $g: G_{1}^{\wedge}$ $\rightarrow G_{2}$. Then we can define the morphism $\varphi: \mathbb{G}_{1} \rightarrow \mathbb{G}_{2}$, defined by

$$
\varphi(w) \stackrel{\text { def }}{=} \begin{cases}g(w) & \text { if } w \in V\left(G_{1}\right) \cup E\left(G_{1}\right) \\ g\left(e_{1}\right) \ldots g\left(e_{n}\right) & \text { if } w=e_{1} \ldots e_{n} \in F P_{r}\left(G^{\wedge}\right), \\ \emptyset_{2} & \text { with } e_{1}, \ldots, e_{n} \in E\left(G^{\wedge}\right), \text { for } n>1\end{cases}
$$

where $\emptyset_{1}$ and $\emptyset_{2}$ are the empty word in $\mathbb{G}_{1}$ and $\mathbb{G}_{2}$, respectively. We can easily check that $\varphi$ is a groupoid-isomorphism, preserving the admissibility on $\mathbb{G}_{1}$ to that on $\mathbb{G}_{2}$.

Let $\mathcal{X}=(\mathcal{X}, \mathcal{Y}, s, r)$ be a groupoid. We say that this groupoid $\mathcal{X}$ acts on a set $Y$ if there exists a groupoid action $\pi$ of $\mathcal{X}$ such that $\pi(x): Y \rightarrow Y$ is a well-determined function, for all $x \in \mathcal{X}$. Sometimes, we call the set $Y$, a $\mathcal{X}$-set. We are interested in the case where a $\mathcal{X}$-set $Y$ is a Hilbert space. The nicest example of a groupoid action acting on a Hilbert space is a graph-representation defined and observed in [10].

Definition 2.1. Let $\mathcal{X}_{1} \subset \mathcal{X}_{2}$ be a subset, where $\mathcal{X}_{2}=\left(\mathcal{X}_{2}, \mathcal{Y}_{2}, s, r\right)$ is a groupoid, and assume that $\mathcal{X}_{1}=\left(\mathcal{X}_{1}, \mathcal{Y}_{1}, s, r\right)$, itself, is a groupoid, where $\mathcal{Y}_{1}=\mathcal{X}_{2} \cap \mathcal{Y}_{2}$. Then we say that the groupoid $\mathcal{X}_{1}$ is a subgroupoid of $\mathcal{X}_{2}$. 
Recall that we say that a countable directed graph $G_{1}$ is a full-subgraph of a countable directed graph $G_{2}$, if

$$
E\left(G_{1}\right) \subseteq E\left(G_{2}\right)
$$

and

$$
V\left(G_{1}\right)=\left\{v \in V\left(G_{1}\right): e=v e \text { or } e=e v, \forall e \in E\left(G_{1}\right)\right\} .
$$

Remark the difference between full-subgraphs and subgraphs: We say that $G_{1}^{\prime}$ is a subgraph of $G_{2}$, if

and

$$
V\left(G_{1}^{\prime}\right) \subseteq V\left(G_{2}\right)
$$

$$
E\left(G_{1}^{\prime}\right)=\left\{e \in E\left(G_{2}\right): e=v_{1} \text { e } v_{2}, \text { for } v_{1}, v_{2} \in V\left(G_{1}^{\prime}\right)\right\}
$$

We can see that the graph groupoid $\mathbb{G}_{1}$ of $G_{1}$ is a subgroupoid of the graph groupoid $\mathbb{G}_{2}$ of $G_{2}$, whenever $G_{1}$ is a full-subgraph of $G_{2}$.

Proposition 2.2. Let $G_{1}$ and $G_{2}$ be countable directed graphs with their graph groupoids $\mathbb{G}_{1}$ and $\mathbb{G}_{2}$, respectively. If $G_{1}$ is a full-subgraph of $G_{2}$, then $\mathbb{G}_{1}$ is a subgroupoid of $\mathbb{G}_{2}$

Proof. Since $G_{1}$ is a full-subgraph of $G_{2}$, the shadowed graph $G_{1}$ of $G_{1}$ is also a full-subgraph of the shadowed graph $G_{2}$ of $G_{2}$. Therefore, the graph groupoid $\mathbb{G}_{1}$ of $G_{1}$ is a subset of the graph groupoid $\mathbb{G}_{2}$ of $G_{2}$. Clearly, $\mathbb{G}_{1}$, itself, is a graph groupoid, and hence it is a groupoid. Therefore, $\mathbb{G}_{1}$ is a subgroupoid of $\mathbb{G}_{2}$

\subsection{Automata and Fractal Groups.}

Automata Theory is the study of abstract machines, and we are using it in the formulation given by von Neumann. It is related to the theory of formal languages. In fact, automata may be thought of as the class of formal languages they are able to recognize. In von Neumann's version, an automaton is a finite state machine (FSM). i.e., a machine with input of symbols, transitions through a series of states according to a transition function (often expressed as a table). The transition function tells the automata which state to go to next, given a current state and a current symbol. The input is read sequentially, symbol by symbol, for example as a tape with a word written on it, registered by the head of the automaton; the head moves forward over the tape one symbol at a time. Once the input is depleted, the automaton stops. Depending on the state in which the automaton stops, it is said that the automaton either accepts or rejects the input. The set of all the words accepted by the automaton is called the language of the automaton. For the benefit 
for the readers, we offer the following references for the relevant part of Automata Theory: [1], [33], [34], [35], [48] and [49].

Let the quadruple $\mathcal{A}=\langle D, Q, \varphi, \psi>$ be given, where $D$ and $Q$ are sets and

$$
\varphi: D \times Q \rightarrow Q \quad \text { and } \quad \psi: D \times Q \rightarrow D
$$

are maps. We say that $D$ and $Q$ are the (finite) alphabet and the state set of $\mathcal{A}$, respectively and we say that $\varphi$ and $\psi$ are the output function and the state transition function, respectively. In this case, the quadruple $\mathcal{A}$ is called an automaton. If the map $\psi(\bullet, q)$ is bijective on $D$, for any fixed $q \in Q$, then we say that the automaton $A$ is invertible. Similarly, if the map $\varphi(x, \bullet)$ is bijective on $Q$, for any fixed $x \in$ $D$, then we say that the automaton $\mathcal{A}$ is reversible. If the automaton $\mathcal{A}$ is both invertible and reversible, then $\mathcal{A}$ is said to be bi-reversible.

To help visualize the use of automata, a few concrete examples may help. With some oversimplification, they may be drawn from the analysis and synthesis of input / output models in Engineering, often referred to as black box diagram: excitation variables, response variables, and intermediate variables (e.g., see [52] and [53]).

In our presentation above, the $D$ (the chosen finite alphabet) often takes different forms on the side of input $D_{i}$ and output $D_{o}$. In popular automata that models stimuli of organisms, the three sets input $D_{i}$, output $D_{o}$, and the state set $Q$, could be as in the following prtotypical three examples:

Example 2.1. Models stimuli of organisms:

$$
\begin{aligned}
D_{i} & =\{\text { positive stimulus, negative stimulus }\}, \\
D_{o} & =\{\text { reaction, no reaction }\}, \\
\text { and } & \\
Q & =\left\{\begin{array}{c}
\text { reaction to last positive stimulus, } \\
\text { no reaction to last positive stimulus }
\end{array}\right\} .
\end{aligned}
$$

Example 2.2. In a control model for say a steering mechanism in a vehicle:

$$
\begin{aligned}
D_{i} & =\{\text { right, left }\}, \\
D_{o} & =\{\text { switch on, switch off }\} \text { or }\{\text { lamp on, lamp off }\} \\
\text { and } & \\
Q & =\left\{\begin{array}{c}
\text { right-turning direction signal } \\
\text { left-turning direction signal }
\end{array}\right\} .
\end{aligned}
$$

Example 2.3. In a model for quantization in Signal Processing: 


$$
D=D_{i}=D_{o}=\left\{\begin{array}{c}
\text { assignments from a bit alphabet, } \\
\text { with the bits referring to the value } \\
\text { of pulses-in and pulses-out in a } \\
\text { signal processing algorithm }
\end{array}\right\},
$$

for example, on a discrete multiresolution (e.g., [50]), and

$$
Q=\begin{aligned}
& \text { a subset of the Cartesian product of } \\
& \text { copies of } D, \text { fixing finite number of } \\
& \text { times, i.e., } D \times \ldots \times D
\end{aligned}
$$

Recently, various algebraists have studied automata and the corresponding automata groups (Also, see [1], [20], [33] and [35]). We will consider a certain special case, where $Q$ is a free semigroupoid of a shadowed graph.

Roughly speaking, a undirected tree is a connected simplicial graph without loop finite paths. Recall that a (undirected) graph is simplicial, if the graph has neither loop-edges nor multi-edges. A directed tree is a connected simplicial graph without loop finite paths, with directed edges (See Section 5.1, more in detail). In particular, we say that a directed tree $\mathcal{T}_{n}$ is a $n$-regular tree, if $\mathcal{T}_{n}$ is rooted and oneflowed infinite directed tree, having the same out-degrees $n$, for all vertices (Also, see Section 5.1, for details). For example, the 2-regular tree $\mathcal{T}_{2}$ can be depicted by

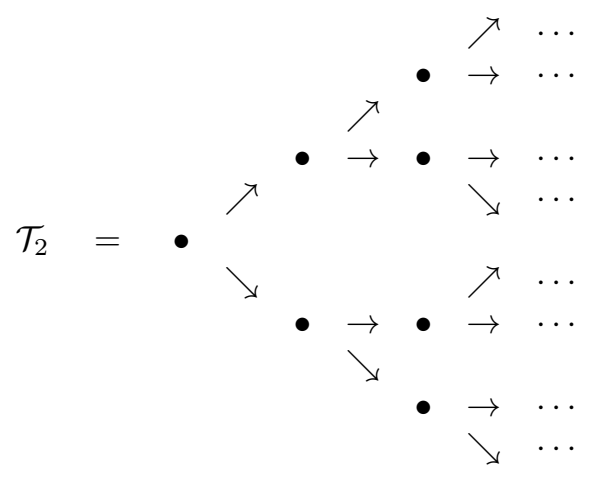

Let $\mathcal{A}=\langle D, Q, \varphi, \psi\rangle$ be an automaton with $|D|=n$. Then, we can construct automata actions of $\mathcal{A}$ on $\mathcal{T}_{n}$. Let's fix $q \in Q$. Then the action of $\mathcal{A}_{q}$ is defined on the finite words $D_{*}$ of $D$ by

$$
\mathcal{A}_{q}(x) \stackrel{\text { def }}{=} \varphi(x, q), \text { for all } x \in D
$$

and recursively,

$$
\mathcal{A}_{q}\left(\left(x_{1}, x_{2}, \ldots, x_{m}\right)\right)=\varphi\left(x_{1}, \mathcal{A}_{q}\left(x_{2}, \ldots, x_{m}\right)\right),
$$

for all $\left(x_{1}, \ldots, x_{m}\right) \in D_{*}$, where 


$$
D_{*} \stackrel{\text { def }}{=} \cup_{m=1}^{\infty}\left(\left\{\begin{array}{l|l}
\left(x_{1}, \ldots, x_{m}\right) \in D^{m} & \begin{array}{c}
x_{k} \in D, \text { for all } \\
k=1, \ldots, n
\end{array}
\end{array}\right\}\right) .
$$

Then the automata actions $\mathcal{A}_{q}$ 's are acting on the $n$-regular tree $\mathcal{T}_{n}$. In other words, all images of automata actions are regarded as an elements in the free semigroupoid $\mathbb{F}^{+}\left(\mathcal{T}_{n}\right)$ of the $n$-regular tree. i.e.,

$$
V\left(\mathcal{T}_{n}\right) \supseteq D_{*}
$$

and its edge set

$$
E\left(\mathcal{T}_{n}\right) \supseteq\left\{\mathcal{A}_{q}(x): x \in D, q \in Q\right\} .
$$

This makes us to illustrate how the automata actions work.

Let $\mathcal{C}=\left\{\mathcal{A}_{q}: q \in Q\right\}$ be the collection of automata actions of the given automaton $\mathcal{A}=\langle D, Q, \varphi, \psi\rangle$. Then we can create a group $G(\mathcal{A})$ generated by the collection $\mathcal{C}$. This group $G(\mathcal{A})$ is called the automata group generated by $\mathcal{A}$. The generator set $\mathcal{C}$ of $G(\mathcal{A})$ acts fully on the $|D|$-regular tree $\mathcal{T}_{|D|}$, we say that this group $G(\mathcal{A})$ is a fractal group. There are many ways to define fractal groups, but we define them in the sense of automata groups. (See [1] and [35]. In fact, in [35], Batholdi, Grigorchuk and Nekrashevych did not define the term "fractal", but they provide the fractal properties.)

Now, we will define a fractal group more precisely (Also see [1]). Let $\mathcal{A}$ be an automaton and let $\Gamma=G(\mathcal{A})$ be the automata group generated by the automata actions acting on the $n$-regular tree $\mathcal{T}_{n}$, where $n$ is the cardinality of the alphabet of $\mathcal{A}$. By $S t_{\Gamma}(k)$, denote the subgroup of $\Gamma=G(\mathcal{A})$, consisting of those elements of $\Gamma$, acting trivially on the $k$-th level of $\mathcal{T}_{n}$, for all $k \in \mathbb{N} \cup\{0\}$.

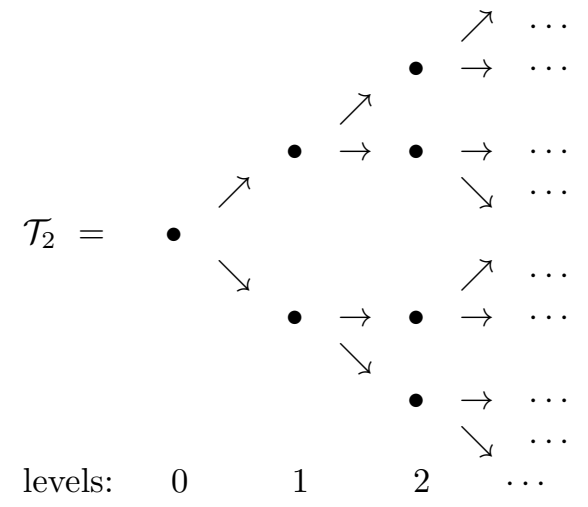

Analogously, for a vertex $u$ in $\mathcal{T}_{n}$, define $S t_{\Gamma}(u)$ by the subgroup of $\Gamma$, consisting of those elements of $\Gamma$, acting trivially on $u$. Then 


$$
S t_{\Gamma}(k)={ }_{u \text { : vertices of the } k \text {-th level of } \mathcal{T}_{n}}\left(S t_{\Gamma}(u)\right)
$$

For any vertex $u$ of $\mathcal{T}_{n}$, we can define the algebraic projection $p_{u}: S t_{\Gamma}(u) \rightarrow \Gamma$.

Definition 2.2. Let $\Gamma=G(\mathcal{A})$ be the automata group given as above. We say that this group $\Gamma$ is a fractal group if, for any vertex $u$ of $\mathcal{T}_{n}$, the image of the projection $p_{u}\left(S t_{\Gamma}(u)\right)$ is group-isomorphic to $\Gamma$, after the identification of the tree $\mathcal{T}_{n}$ with its subtree $\mathcal{T}_{u}$ with the root $u$.

For instance, if $u$ is a vertex of the 2-regular tree $\mathcal{T}_{2}$, then we can construct a subtree $\mathcal{T}_{u}$, as follows:

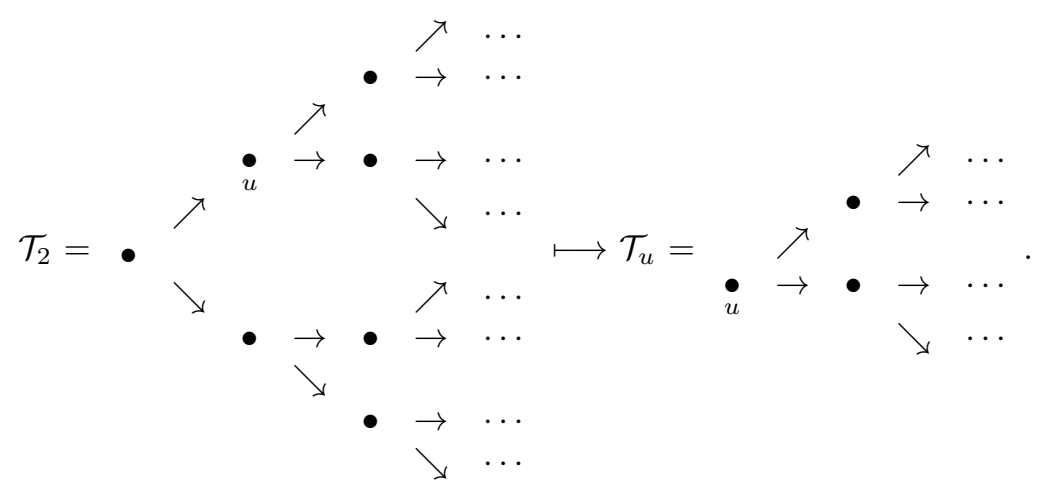

As we can check, the graphs $\mathcal{T}_{2}$ and $\mathcal{T}_{u}$ are graph-isomorphic. So, the above definition shows that if the automata actions $\mathcal{A}_{q}$ 's of $\mathcal{A}$ are acting fully on $\mathcal{T}_{n}$, then the automata group $G(\mathcal{A})$ is a fractal group. There are lots of famous fractal groups, but we introduce the following example, for our purpose.

Example 2.4. Let $\mathcal{A}=\left\langle X_{2 n}, F_{n}, \varphi, \psi>\right.$ be an automaton, where $F_{n}$ is the free group with its generator set $X_{2 n}=\left\{g_{1}^{ \pm 1}, \ldots, g_{n}^{ \pm 1}\right\}$. Then the automata group $G(\mathcal{A})$ is group-isomorphic to $F_{n}$. It is easy to check that all elements in $F_{n}$ acts fully on the $2 n$-regular tree $\mathcal{T}_{2 n}$, and hence $G(\mathcal{A})$ is a fractal group. For example, if $n=2$, then we can get the following 0 -th and 1-st levels of $\mathcal{T}_{4}$ :

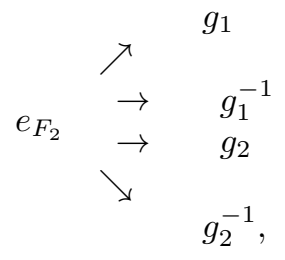

where $e_{F_{2}}$ is the group-identity of $F_{2}=<g_{1}, g_{2}>$. 


\subsection{Free Probability.}

Early versions of free probability models were motivated in part by finitely correlated states which were built mathematically to realize quantum spin chains; see e.g., [47], [54], [55], [56], and [57]; the purpose being to analyze phase transition: Again large composite systems are built from finite alphabets, with recursive algorithms run on finite-state machines, with the finite data representing for example spin variables on a lattice, in the simplest case, the lattice may be the set of integers with its usual order, and the forward correlations depending on a finite number of states going back in the state iterations. To make this precise, we consider a pair of von Neumann algebras $B \subset A$, and with a prescribed conditional expectation $E$ from $A$ onto $B$, mapping the unit of $A$ to that of $B$. The free construction of states on infinite models built on the pair $B \subset A$ is then done by repeating a prescribed automaton which we now proceed to describe.

Let $B \subset A$ be von Neumann algebras with $1_{B}=1_{A}$ and assume that there is a conditional expectation $E_{B}: A \rightarrow B$ satisfying that (i) $E_{B}$ is a (C्C-)linear map, (ii) $E_{B}(b)=b$, for all $b \in B$, (iii) $E_{B}\left(b_{1} a b_{2}\right)=b_{1} E_{B}(a) b_{2}$, for all $b_{1}, b_{2} \in B$ and $a \in$ $A$, (iv) $E_{B}$ is continuous under the given topologies of $A$ and $B$, and (v) $E_{B}\left(a^{*}\right)=$ $E_{B}(a)^{*}$ in $B$, for all $a \in A$. The algebraic pair $\left(A, E_{B}\right)$ is said to be a $B$-valued $W^{*}$ probability space. Every operator in $\left(A, E_{B}\right)$ is called a $B$-valued (free) random variable. Any $B$-valued random variables have their $B$-valued free distributional data: $B$-valued $*$-moments and $B$-valued $*$-cumulants of them. Suppose $a_{1}, \ldots, a_{s}$ are $B$-valued random variables in $\left(A, E_{B}\right)$, where $s \in \mathbb{N}$. The $\left(i_{1}, \ldots, i_{n}\right)$-th joint $B$-valued $*$-moments of $a_{1}, \ldots, a_{s}$ are defined by

$$
E_{B}\left(\left(b_{1} a_{i_{1}}^{r_{i_{1}}}\right)\left(b_{2} a_{i_{2}}^{r_{i_{2}}}\right) \ldots\left(b_{n} a_{i_{n}}^{r_{i_{n}}}\right)\right)
$$

and the $\left(j_{1}, \ldots, j_{k}\right)$-th joint $B$-valued $*$-cumulants of $a_{1}, \ldots, a_{s}$ are defined by

$$
k_{k}^{B}\left(\left(b_{1} a_{j_{1}}^{r_{i_{1}}}\right), \ldots,\left(b_{k} a_{j_{k}}^{r_{j_{k}}}\right)\right)=\sum_{\pi \in N C(k)}^{(N)} E_{B: \pi}\left(b_{1} a_{j_{1}}^{r_{j_{1}}}, \ldots, b_{k} a_{j_{k}}^{r_{j_{k}}}\right) \mu\left(\pi, 1_{k}\right)
$$

for all $\left(i_{1}, \ldots, i_{n}\right) \in\{1, \ldots, s\}^{n}$ and for all $\left(j_{1}, \ldots, j_{k}\right) \in\{1, \ldots, s\}^{k}$, for $n, k \in \mathbb{N}$, where $b_{j} \in B$ are arbitrary and $r_{i_{1}}, \ldots, r_{i_{n}}, r_{j_{1}}, \ldots, r_{j_{k}} \in\{1, *\}$ and $N C(k)$ is the lattice of all noncrossing partitions with its minimal element $0_{k}=\{(1),(2), \ldots,(k)\}$ and its maximal element $1_{k}=\{(1,2, \ldots, k)\}$, for all $k \in \mathbb{N}$, and $\mu$ is the Moebius functional in the incidence algebra $\mathcal{I}$. Here, $E_{B: \pi}(\ldots)$ is the partition-depending $B$-valued moment. For example, if $\pi=\{(1,4),(2,3),(5)\}$ in $N C(5)$, then

$$
E_{B: \pi}\left(a_{1}, a_{2}, a_{3}, a_{4}, a_{5}\right)=E_{B}\left(a_{1} E_{B}\left(a_{2} a_{3}\right) a_{4}\right) E_{B}\left(a_{5}\right) .
$$


Recall that the lattice $N C(n)$ of all noncrossing partitions over $\{1, \ldots, n\}$ has its partial ordering " $\leq$ ",

$$
\pi \leq \theta \stackrel{\text { def }}{\Longleftrightarrow} \text { for each block } V \text { in } \pi, \exists \text { a block } B \text { in } \theta \text { s.t.,. } V \subseteq B,
$$

for $\pi, \theta \in N C(n)$, where " $\subseteq$ " means the usual set inclusion, for all $n \in \mathbb{N}$. Also recall that the incidence algebra $\mathcal{I}$ is the collection of all functionals

$$
\xi: \cup_{n=1}^{\infty}(N C(n) \times N C(n)) \rightarrow \mathbb{C}
$$

satisfying that $\xi(\pi, \theta)=0$, whenever $\pi>\theta$, with the usual function addition $(+)$ and the convolution $(*)$ defined by

$$
\left(\xi_{1} * \xi_{2}\right)(\pi, \theta) \stackrel{\text { def }}{=} \sum_{\pi \leq \sigma \leq \theta}^{(N)} \xi_{1}(\pi, \sigma) \xi_{2}(\sigma, \theta)
$$

for all $\xi_{1}, \xi_{2} \in \mathcal{I}$. If we define the zeta functional $\zeta \in \mathcal{I}$ by

$$
\zeta(\pi, \theta)=1, \text { for all } \pi \leq \theta \text { in } N C(n) \text {, for all } n \in \mathbb{N},
$$

then its convolution-inverse in $\mathcal{I}$ is the Moebius functional $\mu$. Thus the Moebius functional $\mu$ satisfies that

$$
\mu\left(0_{n}, 1_{n}\right)=(-1)^{n-1} c_{n-1} \text { and } \sum_{\pi \in N C(n)}^{(N)} \mu\left(\pi, 1_{n}\right)=0
$$

where $c_{k} \stackrel{\text { def }}{=} \frac{1}{k+1}\left(\begin{array}{c}2 k \\ k\end{array}\right)$ is the $k$-th Catalan number, for all $k \in \mathbb{N}$ (See [21]).

The $B$-valued freeness on $\left(A, E_{B}\right)$ is characterized by the $B$-valued $*$-cumulants. Let $A_{1}$ and $A_{2}$ be $W^{*}$-subalgebras of $A$ having their common $W^{*}$-subalgebra $B$. We say that $A_{1}$ and $A_{2}$ are free over $B$ in $\left(A, E_{B}\right)$ if all mixed $B$-valued $*$-cumulants of $A_{1}$ and $A_{2}$ vanish. The subsets $X_{1}$ and $X_{2}$ of $A$ are said to be free over $B$ in $(A$, $\left.E_{B}\right)$ if the $W^{*}$-subalgebras $v N\left(X_{1}, B\right)$ and $v N\left(X_{2}, B\right)$ of $A$ are free over $B$ in $(A$, $\left.E_{B}\right)$, where $v N\left(S_{1}, S_{2}\right)$ means the von Neumann algebra generated by sets $S_{1}$ and $S_{2}$. Similarly, we say that the $B$-valued random variables $x$ and $y$ are free over $B$ in $\left(A, E_{B}\right)$ if the subsets $\{x\}$ and $\{y\}$ are free over $B$ in $\left(A, E_{B}\right)$ (Also See [21]).

Let $A_{1}$ and $A_{2}$ be $W^{*}$-subalgebra of $A$ containing their common $W^{*}$-subalgebra $B$, and assume that they are free over $B$ in $\left(A, E_{B}\right)$. Then we can construct a $W^{*}$-algebra $v N\left(A_{1}, A_{2}\right)$ of $A$ generated by $A_{1}$ and $A_{2}$. We denote it by $A_{1} *_{B} A_{2}$. Suppose there exists a family $\left\{A_{i}: i \in \Lambda\right\}$ of $W^{*}$-subalgebras of $A$ containing their common $W^{*}$-subalgebra $B$. If they generate $A$ and if they are free over $B$ from each other in $\left(A, E_{B}\right)$, then the von Neumann algebra $A$ is the $B$-valued free product algebra $\underset{i \in \Lambda}{*} A_{i}$. 
Suppose a von Neumann algebra $A$ is a $B$-free product algebra $\underset{i \in \Lambda}{*} A_{i}$. Then $A$ is Banach-space isomorphic to the Banach space

$$
B \oplus\left(\oplus_{n=1}^{\infty}(\underbrace{\oplus}_{i_{1} \neq i_{2}, i_{2} \neq i_{3}, \ldots, i_{n-1} \neq i_{n}}\left(\begin{array}{lllll}
A_{i_{1}}^{o} & \otimes_{B} & \ldots & \otimes_{B} & A_{i_{n}}^{o}
\end{array}\right))\right)
$$

with

$$
A_{i_{j}}^{o} \stackrel{\text { def }}{=} A_{i_{j}} \ominus B, \text { for all } j=1, \ldots, n,
$$

where $\otimes_{B}$ is the $B$-valued tensor product.

\subsection{Graph Groupoids and Right Graph Von Neumann algebras.}

Let $G$ be a countable directed graph with its vertex set $V(G)$ and its edge set $E(G)$. Denote the set of all finite paths of $G$ by $F P(G)$. Clearly, the edge set $E(G)$ is contained in $F P(G)$. Let $w$ be a finite path in $F P(G)$. Then it is represented as a word in $E(G)$, depending on the directions of edges. If $e_{1}, \ldots, e_{n}$ are connected directed edges in the order $e_{1} \rightarrow e_{2} \rightarrow \ldots \rightarrow e_{n}$, for $n \in \mathbb{N}$, then we can express $w$ by $e_{1} \ldots e_{n}$ in $F P(G)$. If there exists a finite path $w=e_{1} \ldots e_{n}$ in $F P(G)$, where $n \in \mathbb{N} \backslash\{1\}$, we say that the directed edges $e_{1}, \ldots, e_{n}$ are admissible. The length $|w|$ of $w$ is defined to be $n$, which is the cardinality of the admissible edges generating $w$. Also, we say that finite paths $w_{1}=e_{11} \ldots e_{1 k_{1}}$ and $w_{2}=e_{21} \ldots e_{2 k_{2}}$ are admissible, if $w_{1} w_{2}=e_{11} \ldots e_{1 k_{1}} e_{21} \ldots e_{2 k_{2}}$ is again in $F P(G)$, where $e_{11}, \ldots$, $e_{1 k_{1}}, e_{21}, \ldots, e_{2 k_{2}} \in E(G)$. Otherwise, we say that $w_{1}$ and $w_{2}$ are not admissible. Suppose that $w$ is a finite path in $F P(G)$ with its initial vertex $v_{1}$ and its terminal vertex $v_{2}$. Then we write $w=v_{1} w$ or $w=w v_{2}$ or $w=v_{1} w v_{2}$, for emphasizing the initial vertex of $w$, respectively the terminal vertex of $w$, respectively both the initial vertex and the terminal vertex of $w$. Suppose $w=v_{1} w v_{2}$ in $F P(G)$ with $v_{1}, v_{2} \in V(G)$. Then we also say that $\left[v_{1}\right.$ and $w$ are admissible] and $\left[w\right.$ and $v_{2}$ are admissible]. Notice that even though the elements $w_{1}$ and $w_{2}$ in $V(G) \cup F P(G)$ are admissible, $w_{2}$ and $w_{1}$ are not admissible, in general. For instance, if $e_{1}=v_{1}$ $e_{1} v_{2}$ is an edge with $v_{1}, v_{2} \in V(G)$ and $e_{2}=v_{2} e_{2} v_{3}$ is an edge with $v_{3} \in V(G)$ such that $v_{3} \neq v_{1}$, then there is a finite path $e_{1} e_{2}$ in $F P(G)$, but there is no finite path $e_{2} e_{1}$.

The free semigroupoid $\mathbb{F}^{+}(G)$ of $G$ is defined by a set

$$
\mathbb{F}^{+}(G)=\{\emptyset\} \cup V(G) \cup F P(G)
$$

with its binary operation $(\cdot)$ on $\mathbb{F}^{+}(G)$, defined by 


$$
\left(w_{1}, w_{2}\right) \mapsto w_{1} \cdot w_{2}= \begin{cases}w_{1} & \text { if } w_{1}=w_{2} \text { in } V(G) \\ w_{1} & \text { if } w_{1} \in F P(G), w_{2} \in V(G) \text { and } w_{1}=w_{1} w_{2} \\ w_{2} & \text { if } w_{1} \in V(G), w_{2} \in F P(G) \text { and } w_{2}=w_{1} w_{2} \\ w_{1} w_{2} & \text { if } w_{1}, w_{2} \text { in } F P(G) \text { and } w_{1} w_{2} \in F P(G) \\ \emptyset & \text { otherwise, }\end{cases}
$$

where $\emptyset$ is the empty word in $V(G) \cup E(G)$. (Sometimes, the free semigroupoid $\mathbb{F}^{+}(G)$ of a certain graph $G$ does not contain the empty word $\emptyset$. For instance, the free semigroupoid of the one-vertex-multi-loop-edge graph does not have the empty word. But, in general, the empty word $\emptyset$ is contained in the free semigroupoid, whenever $|V(G)| \geq 2$. So, if there is no confusion, then we usually assume that the empty word is contained in free semigroupoids.) This binary operation $(\cdot)$ on $\mathbb{F}^{+}(G)$ is called the admissibility. i.e., the algebraic structure $\left(\mathbb{F}^{+}(G), \cdot\right)$ is the free semigroupoid of $G$. For convenience, we denote $\left(\mathbb{F}^{+}(G), \cdot\right)$ simply by $\mathbb{F}^{+}(G)$.

For the given countable directed graph $G$, we can define a new countable directed graph $G^{-1}$ which is the opposite directed graph of $G$, with

$$
V\left(G^{-1}\right)=V(G) \quad \text { and } \quad E\left(G^{-1}\right)=\left\{e^{-1}: e \in E(G)\right\}
$$

where $e^{-1} \in E\left(G^{-1}\right)$ is the opposite directed edge of $e \in E(G)$, called the shadow of $e \in E(G)$. i.e., if $e=v_{1} e v_{2}$ in $E(G)$ with $v_{1}, v_{2} \in V(G)$, then $e^{-1}=v_{2} e^{-1} v_{1}$ in $E\left(G^{-1}\right)$ with $v_{2}, v_{1} \in V\left(G^{-1}\right)=V(G)$. This new directed graph $G^{-1}$ is said to be the shadow of $G$. It is trivial that $\left(G^{-1}\right)^{-1}=G$. This relation shows that the admissibility on the shadow $G^{-1}$ is oppositely preserved by that on $G$.

A new countable directed graph $G^{\wedge}$ is called the shadowed graph of $G$ if it is a directed graph with

and

$$
V\left(G^{\wedge}\right)=V(G)=V\left(G^{-1}\right)
$$

$$
E\left(G^{\wedge}\right)=E(G) \cup E\left(G^{-1}\right) .
$$

Definition 2.3. Let $G$ be a countable directed graph and $G^{\wedge}$, the shadowed graph of $G$, and let $\mathbb{F}^{+}\left(G^{\wedge}\right)$ be the free semigroupoid of $G^{\wedge}$. Define the reduction (RR) on $\mathbb{F}^{+}\left(G^{\wedge}\right)$ by

$$
w w^{-1}=v \quad \text { and } \quad w^{-1} w=v^{\prime},
$$

whenever $w=v w v^{\prime}$ in $F P\left(G^{\wedge}\right)$, with $v, v^{\prime} \in V\left(G^{\wedge}\right)$. The set $\mathbb{F}^{+}\left(G^{\wedge}\right)$ with this reduction $(R R)$ is denoted by $\mathbb{F}_{r}^{+}\left(G^{\wedge}\right)$. And this set $\mathbb{F}_{r}^{+}\left(G^{\wedge}\right)$ with the inherited admissibility $(\cdot)$ from $\mathbb{F}^{+}\left(G^{\wedge}\right)$ is called the graph groupoid of $G$. Denote $\left(\mathbb{F}_{r}^{+}\left(G^{\wedge}\right)\right.$, .) of $G$ by $\mathbb{G}$.

The graph groupoid $\mathbb{G}$ of $G$ is a (categorial) groupoid with its base $V\left(G^{\wedge}\right)$. Define the reduced finite path set $F P_{r}\left(G^{\wedge}\right)$ of $\mathbb{G}$ by 


$$
F P_{r}\left(G^{\wedge}\right) \stackrel{\text { def }}{=} \mathbb{F}_{r}^{+}\left(G^{\wedge}\right) \backslash\left(V\left(G^{\wedge}\right) \cup\{\emptyset\}\right)
$$

The subset $F P_{r}\left(G^{\wedge}\right)$ of $\mathbb{G}$ is called the reduced finite path set of $G$. Let $w_{1}$ and $w_{2}$ be reduced finite paths in $F P_{r}\left(G^{\wedge}\right) \subset \mathbb{G}$. We will use the same notation $w_{1} w_{2}$ for the product of $w_{1}$ and $w_{2}$ in $\mathbb{G}$. But we have to keep in mind that the product $w_{1} w_{2}$ in the graph groupoid $\mathbb{G}$ is different from the product $w_{1} w_{2}$ in the free semigroupoid $\mathbb{F}^{+}\left(G^{\wedge}\right)$. Suppose $e_{1}$ and $e_{2}$ are edges in $E\left(G^{\wedge}\right)$ and assume that they are admissible, and hence $e_{1} e_{2}$ is a finite path in $F P\left(G^{\wedge}\right)$. Then the product $e_{1}$ $e_{2} e_{2}^{-1}$ of $e_{1} e_{2}$ and $e_{2}^{-1}$ is the length-3 finite path in $F P\left(G^{\wedge}\right) \subset \mathbb{F}^{+}\left(G^{\wedge}\right)$, but the product $e_{1} e_{2} e_{2}^{-1}$ of them is $e_{1}\left(e_{2} e_{2}^{-1}\right)=e_{1}$ in $F P_{r}\left(G^{\wedge}\right) \subset \mathbb{G}$.

Now, we will define and consider certain operators on graph Hilbert spaces. As we defined and observed, we can regard all elements in $\mathbb{G}$ as reduced words in $E\left(G^{\wedge}\right)$, under the admissibility with the reduction $(\mathrm{RR})$.

Definition 2.4. Let $G$ be a countable directed graph and let $\mathbb{G}$ be the corresponding graph groupoid. Define the Hilbert space $H_{G}$ of $G$ by

$$
H_{G} \stackrel{\text { def }}{=} \underset{w \in F P_{r}\left(G^{\wedge}\right)}{\oplus}\left(\mathbb{C} \xi_{w}\right)
$$

with its Hilbert basis $\mathcal{B}_{H_{G}}=\left\{\xi_{w}: w \in F P_{r}\left(G^{\wedge}\right)\right\}$ in $H_{G}$, where $F P_{r}\left(G^{\wedge}\right)$ is the reduced finite path set.

We have the following multiplication rule on $H_{G}$ :

$$
\xi_{w_{1}} \xi_{w_{2}}= \begin{cases}\xi_{w_{1} w_{2}} & \text { if } w_{1} w_{2} \neq \emptyset \\ \xi_{\emptyset}=0_{H_{G}} & \text { otherwise }\end{cases}
$$

for all $\xi_{w_{1}}, \xi_{w_{2}} \in \mathcal{B}_{H_{G}}$. Suppose $w_{1}=w$ and $w_{2}=w^{-1}$ in $F P_{r}\left(G^{\wedge}\right)$. Then, by the above multiplication rule, we can have $\xi_{w} \xi_{w^{-1}}=\xi_{w w^{-1}}$ and $w w^{-1}$ is a vertex in $V\left(G^{\wedge}\right)$. So, we can determine $\xi_{v} \in H_{G}$, for all $v \in V\left(G^{\wedge}\right)$. And hence, we can extend the above multiplication rule to the case where $w_{1}, w_{2} \in \mathbb{G}$. This multiplication rule let us define multiplication operators on $H_{G}$.

Definition 2.5. An operator $R_{w}$ on $H_{G}$ is defined by the right multiplication operator with its symbol $\xi_{w}$ on $H_{G}$, for $w \in \mathbb{G}$. i.e.,

$$
R_{w} \xi_{w^{\prime}} \stackrel{\text { def }}{=} \begin{cases}\xi_{w^{\prime}} \xi_{w}=\xi_{w^{\prime} w} & \text { if } w^{\prime} w \neq \emptyset \\ \xi_{\emptyset}=0_{H_{G}} & \text { otherwise }\end{cases}
$$

for all $w, w^{\prime} \in \mathbb{G}$. The adjoint $R_{w}^{*}$ of $R_{w}$ is defined by $R_{w}^{*}=R_{w^{-1}}$, for all $w \in$ $\mathbb{G}$. 
By definition, the product of two right multiplication operators $R_{w_{1}} R_{w_{2}}$ is the multiplication operator $R_{w_{2} w_{1}}$. i.e.,

$$
R_{w_{1}} R_{w_{2}}=R_{w_{2} w_{1}}, \text { for all } w_{1}, w_{2} \in \mathbb{G}
$$

So, it is easy to check that the multiplication operators $R_{v}$, for all $v \in V\left(G^{\wedge}\right)$, are projections on $H_{G}$, since

$$
R_{v}^{2}=R_{v} R_{v}=R_{v^{2}}=R_{v} \text { and } R_{v}^{*}=R_{v^{-1}}=R_{v} .
$$

Thus we can get that the right multiplication operators $R_{w}$, for all $w \in F P_{r}\left(G^{\wedge}\right)$, are partial isometries. Indeed,

and

$$
R_{w} R_{w}^{*} R_{w}=R_{w w^{-1} w}=R_{w w^{-1}} R_{w}=R_{w}
$$

$$
R_{w}^{*} R_{w} R_{w}^{*}=R_{w^{-1} w w^{-1}}=R_{w^{-1} w} R_{w^{-1}}=R_{w^{-1}}=R_{w}^{*}
$$

for all $w \in F P_{r}\left(G^{\wedge}\right)$.

Remark 2.1. In [10] and [11], we defined the same graph Hilbert space $H_{G}$, for any countable directed graph $G$, and we defined the (left) multiplication operators $L_{w} \in B\left(H_{G}\right)$ by

$$
L_{w} \xi_{w^{\prime}} \stackrel{\text { def }}{=} \begin{cases}\xi_{w w^{\prime}} & \text { if } w w^{\prime} \neq \emptyset \\ 0 & \text { otherwise }\end{cases}
$$

for all $w, w^{\prime} \in \mathbb{G}$. Then they have the similar properties like the right multiplication operators $R_{w}$ on $H_{G}$. i.e., if $w$ is a vertex, then $L_{w}$ is a projection: and if $w$ is a reduced finite path, then $L_{w}$ is a partial isometries on $H_{G}$.

Now, we define certain groupoid actions $\beta$ of the graph groupoid $\mathbb{G}$ on $H_{G}$, called right graph-representations.

Definition 2.6. Let $\mathbb{G}$ be a graph groupoid of a countable directed graph $G$ and let $M$ be a von Neumann algebra acting on a Hilbert space $K$ (i.e., $M \subseteq B(K)$ ). Define a right graph-representation (in short, a right $G$-representation) $\beta: \mathbb{G} \rightarrow$ $B\left(K \otimes H_{G}\right)$, by a nonunital intertwined partial representation satisfying that

$$
\beta_{w}(m) R_{w} R_{w}^{*}=R_{w}^{*} m R_{w}=R_{w^{-1}} m R_{w}
$$

for all $m \in M$ and $w \in \mathbb{G}$, and

$$
\beta_{v}(m)=m, \text { for all } m \in M \text { and } v \in V\left(G^{\wedge}\right) .
$$


In this setting, we can regard $R_{w}$ 's as $1_{K} \otimes R_{w}$ 's on $K \otimes H_{G}$, for all $w \in \mathbb{G}$.

For a fixed von Neumann algebra $M$ and a graph groupoid $\mathbb{G}$, we can construct a groupoid crossed product algebra $M \times_{\beta} \mathbb{G}$, for a fixed right graph-representation $\beta$. This new von Neumann algebra is called a right graph von Neumann algebra induced by $G$ over $M$.

Definition 2.7. Let $M$ be a von Neumann algebra and $\mathbb{G}$, the graph groupoid of a countable directed graph $G$ and let $\beta$ be a right $G$-representation. Define the crossed product $\mathbb{M}_{G}=M \times_{\beta} \mathbb{G}$ of $M$ and $\mathbb{G}$ via $\beta$, by the von Neumann algebra generated by $M$ and $\left\{R_{w}: w \in \mathbb{G}\right\}$ in $B\left(K \otimes H_{G}\right)$, satisfying the above $\beta$-rules on $M$. This von Neumann algebra $\mathbb{M}_{G}$ is called a right graph von Neumann algebra induced by $G$ over $M$.

Remark 2.2. In [10] and [11], we defined a graph von Neumann algebra $\mathbb{M}_{G}^{(\text {left })}$ by the groupoid crossed product $M \times_{\alpha} \mathbb{G}$, where $\alpha: \mathbb{G} \rightarrow B\left(K \otimes H_{G}\right)$ is a $G$ representation satisfying the $\alpha$-rules on $M$ :

$$
\alpha_{w}(m) L_{w} L_{w}^{*}=L_{w}^{*} m L_{w}, \text { for all } w \in F P_{r}\left(G^{\wedge}\right)
$$

and

$$
\alpha_{v}(m)=m, \text { for all } v \in V\left(G^{\wedge}\right),
$$

for all $m \in M$, where $L_{w}$ 's are the (left) multiplication operators, for $w \in \mathbb{G}$ (See the previous remark). Notice that, in fact, the von Neumann algebra $\mathbb{M}_{G}^{(\text {left })}$ is the opposite algebra $\mathbb{M}_{G}^{o p}$ of a right graph von Neumann algebra $\mathbb{M}_{G}=M \times_{\beta} \mathbb{G}$, whenever $\beta=\alpha$ on $M$ (See [13]).

Every operator $x$ in a right graph von Neumann algebra $\mathbb{M}_{G}=M \times_{\beta} \mathbb{G}$ has its expression,

$$
x=\sum_{w \in \mathbb{G}}^{(N)} m_{w} R_{w}, \text { for } m_{w} \in M .
$$

Lemma 2.3. Let $m_{1} R_{w_{1}}, \ldots, m_{n} R_{w_{n}}$ be operators in a right graph von Neumann algebra $\mathbb{M}_{G}$, for $n \in \mathbb{N}$. Then

$$
\begin{aligned}
& \Pi_{k=1}^{n}\left(m_{k} R_{w_{k}}\right) \\
& \quad= \begin{cases}\left(m_{1} m_{2}^{w_{1}^{-1}} m_{3}^{\left(w_{1} w_{2}\right)^{-1}} \ldots m_{n}^{\left(w_{1} w_{2} \ldots w_{n-1}\right)^{-1}}\right) R_{w_{n} \ldots w_{1}} & \text { if } w_{n} \ldots w_{1} \neq \emptyset \\
0_{\mathbb{M}_{G}} & \text { otherwise, }\end{cases}
\end{aligned}
$$


where $m^{w} \stackrel{\text { def }}{=} \beta_{w}(m)$, for all $m \in M$ and $w \in \mathbb{G}$.

\section{5. $M$-Valued Right Graph $W^{*}$-Probability Spaces.}

Let $G$ be a countable directed graph and let $M$ be an arbitrary von Neumann algebra acting on a Hilbert space $K$. In this section, we will define a $M$-diagonal right graph $W^{*}$-probability space $\left(\mathbb{M}_{G}, E\right)$, over its $M$-diagonal subalgebra $\mathbb{D}_{G}$, where $\mathbb{M}_{G}=M \times_{\beta} \mathbb{G}$ is a right graph von Neumann algebra.

Let $v \in V\left(G^{\wedge}\right)$. Then we can define a conditional expectation $E_{v}: \mathbb{M}_{G} \rightarrow M$. $R_{v}$, where $M \cdot R_{v}=\left\{m R_{v}: m \in M\right\}$, by

$$
E_{v}\left(\sum_{w \in \mathbb{G}}^{(N)} m_{w} R_{w}\right)=m_{v} R_{v}
$$

for all $\sum_{w \in \mathbb{G}}^{(N)} m_{w} R_{w} \in \mathbb{M}_{G}$. Notice that each $M \cdot R_{v}$ is a $W^{*}$-subalgebra of $\mathbb{M}_{G}$, which is $*$-isomorphic to $M$, since $R_{v}$ is a projection, for all $v \in V\left(G^{\wedge}\right)$. A pair $\left(\mathbb{M}_{G}, E_{v}\right)$ is a $M$-valued $W^{*}$-probability space, for $v \in V\left(G^{\wedge}\right)$. We call it a vertexdepending (or the $v$-depending) right graph $W^{*}$-probability space over $M$. The conditional expectation $E_{v}$ is said to be a vertex-depending (or the $v$-depending) conditional expectation, for $v \in V\left(G^{\wedge}\right)$. By the very definition, the right graph von Neumann algebra $\mathbb{M}_{G}$ has $\left|V\left(G^{\wedge}\right)\right|$-many vertex-depending $W^{*}$-probability spaces over $M$.

Definition 2.8. By $\mathbb{D}_{G}$, denote a $W^{*}$-subalgebra $\underset{v \in V\left(G^{\wedge}\right)}{\oplus}\left(M \cdot R_{v}\right)$ of $\mathbb{M}_{G}=M$ $\times_{\beta} \mathbb{G}$. This subalgebra $\mathbb{D}_{G}$ is called the $M$-diagonal subalgebra of $\mathbb{M}_{G}$. Define a conditional expectation $E: \mathbb{M}_{G} \rightarrow \mathbb{D}_{G}$, by $\underset{v \in V\left(G^{\wedge}\right)}{\oplus} E_{v}$, where $E_{v}$ 's are the $v$-depending conditional expectations, for all $v \in V\left(G^{\wedge}\right)$. i.e.,

$$
E\left(\sum_{w \in \mathbb{G}}^{(N)} m_{w} R_{w}\right)=\sum_{v \in V\left(G^{\wedge}\right)}^{(N)} m_{v} R_{v}
$$

for all $\sum_{w \in \mathbb{G}}^{(N)} m_{w} R_{w} \in \mathbb{M}_{G}$. The pair $\left(\mathbb{M}_{G}, E\right)$ is called the $M$-diagonal right graph $W^{*}$-probability space over $\mathbb{D}_{G}$.

Definition 2.9. Let $G$ be a countable directed graph and let $\mathbb{G}$ be the graph groupoid of $G$. Define a map $\delta: \mathbb{G} \rightarrow \mathbb{G}$ by mapping $w \in \mathbb{G}$ to the graphical image $\delta(w)$ of $w$, for all $w \in \mathbb{G}$. The map $\delta$ on $\mathbb{G}$ is called the diagram map and the graphical image 
$\delta(w)$ of $w$ is called the diagram of $w$, for all $w \in \mathbb{G}$. We say that the elements $w_{1}$ and $w_{2}$ in $\mathbb{G}$ are diagram-distinct if (i) $w_{1} \neq w_{2}^{-1}$ and (ii) $\delta\left(w_{1}\right) \neq \delta\left(w_{2}\right)$. Suppose that $X_{1}$ and $X_{2}$ are subsets in $\mathbb{G}$. They are said to be diagram-distinct if, for any pair $\left(w_{1}, w_{2}\right)$ in $X_{1} \times X_{2}, w_{1}$ and $w_{2}$ are diagram-distinct.

Let $l$ be a loop edge in $E\left(G^{\wedge}\right)$, and let $w_{1}=l^{k_{1}}$ and $w_{2}=l^{k_{2}}$ in $\mathbb{G}$, for $k_{1} \neq$ $k_{2} \in \mathbb{N}$. Clearly, $w_{1} \neq w_{2}^{-1}$. But the elements $w_{1}$ and $w_{2}$ are not diagram-distinct, because their diagrams $\delta\left(w_{1}\right)$ and $\delta\left(w_{2}\right)$ are identical to the diagram $\delta(l)=l$ of $l$. Suppose either $w_{1}$ or $w_{2}$ is a non-loop finite path such that (i) $w_{1} \neq w_{2}^{-1}$, and (ii) $\delta\left(w_{k}\right)$ 's are not loop finite paths, for $k=1,2$. Then they are diagram-distinct whenever $w_{1}$ and $w_{2}$ are distinct in $\mathbb{G}$.

Theorem 2.4. The subsets $M \cdot R_{w_{1}}$ and $M \cdot R_{w_{2}}$ of a right graph von Neumann algebra $\mathbb{M}_{G}=M \times_{\beta} \mathbb{G}$ are free over $D_{G}$ in $\left(\mathbb{M}_{G}, E\right)$ if and only if $w_{1}$ and $w_{2}$ are diagram-distinct in $\mathbb{G}$, where $M \cdot R_{w} \stackrel{\text { def }}{=}\left\{m L_{w}: m \in M\right\}$, for all $w \in \mathbb{G}$.

We will skip the proof. The readers can find the proof showing that $M \cdot L_{w_{1}}$ and $M \cdot L_{w_{2}}$ are free over $\mathbb{D}_{G}$ in a graph von Neumann algebra $M \times_{\alpha} \mathbb{G}$, in [10] and [11]. By the slight modification, we can prove the above theorem. As corollary of the previous theorem, we can see that if $e_{1}$ and $e_{2}$ are edges in $E(G)\left(\subset E\left(G^{\wedge}\right)\right)$, then $M \cdot R_{e_{1}}$ and $M \cdot R_{e_{2}}$ are free over $\mathbb{D}_{G}$ in $\left(\mathbb{M}_{G}, E\right)$. Indeed, if two edges $e_{1}$ and $e_{2}$ are distinct in $E(G)$, then $e_{1}$ and $e_{2}^{ \pm 2}$ (resp., $e_{2}$ and $e_{1}^{ \pm 1}$ ) are diagram-distinct in $E\left(G^{\wedge}\right) \subset \mathbb{G}$.

Let's consider the subset $\left\{e, e^{-1}\right\}$ of $\mathbb{G}$, for $e \in E(G) \subset E\left(G^{\wedge}\right)$. Then we can define a subset $\mathbb{G}_{e}$ of $\mathbb{G}$, by the collection of all reduced words in $\left\{e, e^{-1}\right\}$. It is easy to check that: if $e$ is a loop edge in $E(G)$, then $\mathbb{G}_{e}$ is a group, which is groupisomorphic to the infinite abelian cyclic group $\mathbb{Z}$, and if $e$ is a non-loop edge in $E(G)$, then $\mathbb{G}_{e}$ is a groupoid $\left\{\emptyset, v_{1}, v_{2}, e, e^{-1}\right\}$, where $e=v_{1} e v_{2}$, with $v_{1}, v_{2}$ $\in V(G)$. Notice that the graph groupoid $\mathbb{G}$ is the reduced free product $\underset{e \in E(G)}{*} \mathbb{G}_{e}$ of $\mathbb{G}_{e}$ 's (in the sense of [10]). Moreover, each $\mathbb{G}_{e}$ can be regarded as a new graph groupoid induced by a one-edge graph $G_{e}$, with $V\left(G_{e}\right)=\left\{v_{1}, v_{2}\right\}$ and $E\left(G_{e}\right)=$ $\left\{e=v_{1} e v_{2}\right\}$, where $v_{1}$ and $v_{2}$ are not necessarily distinct in $V(G)$. Thus we can construct the right graph von Neumann algebras,

$$
\mathbb{M}_{e}=M \times_{\beta_{e}} \mathbb{G}_{e} \text { with }\left.\beta_{e} \stackrel{\text { def }}{=} \beta\right|_{\mathbb{G}_{e}} \text { on } M
$$

for all $e \in E(G)$. Then they are embedded $W^{*}$-subalgebras of $\mathbb{M}_{G}=M \times_{\beta} \mathbb{G}$. Moreover, by the previous theorem, we can have that:

Corollary 2.5. The $W^{*}$-subalgebras $\mathbb{M}_{e}$ 's of $\mathbb{M}_{G}$ are free over $\mathbb{D}_{G}$, from each other, for all $e \in E(G)$, in $\left(\mathbb{M}_{G}, E\right)$.

Definition 2.10. The $W^{*}$-subalgebra $\mathbb{M}_{e}$ of $\mathbb{M}_{G}$ are called $\mathbb{D}_{G}$-free blocks of $\mathbb{M}_{G}$, for all $e \in E(G)$. 
Notice that $\mathbb{M}_{e}$ is identically same as $\mathbb{M}_{e^{-1}}$, for all $e \in E(G)$. So, we can get the following theorem characterizing the $\mathbb{D}_{G}$-free structure of $\left(\mathbb{M}_{G}, E\right)$.

The following three theorems are about the crossed product algebra $\mathbb{M}_{G}$ from Theorem 2.4. The first two theorems both involve the generalized diagonal $\mathbb{D}_{G}$, and they offer decompositions of $\mathbb{M}_{G}$ into certain "blocks". Each of the two decompositions generalizes what is known for finite matrices. The first one (Theorem 2.6 ) is an algebraic decomposition of $\mathbb{M}_{G}$, while the second (Theorem 2.7) offers a canonical decomposition of $\mathbb{M}_{G}$ as a Banach space. Theorem 2.8 in turn proves that the blocks in the decompositions fall in two specific classes.

Theorem 2.6. Let $\left(\mathbb{M}_{G}, E\right)$ be the $M$-diagonal right graph $W^{*}$-probability space over its $M$-diagonal subalgebra $\mathbb{D}_{G}$. Then

$$
\mathbb{M}_{G} \stackrel{* \text {-isomorphic }}{=} \underset{\substack{* \mathbb{D}_{G} \\ e \in E(G)}}{ } \mathbb{M}_{e}
$$

where $\mathbb{M}_{e}$ 's are the $\mathbb{D}_{G}$-free blocks of $\mathbb{M}_{G}$, for all $e \in E(G)$.

Recall that if $\left\{A_{i}\right\}_{i \in I}$ are von Neumann algebras over their common $W^{*}$-subalgebra $B$, then the $B$-free product $\underset{i \in I}{*_{B}} A_{i}$ can be expressed by

$$
B \oplus\left(\oplus_{n=1}^{\infty}\left(\underset{i_{1} \neq i_{2}, i_{2} \neq i_{3}, \ldots, i_{n-1} \neq i_{n} \in I}{\oplus}\left(A_{i_{1}}^{o} \otimes_{B} \ldots \otimes_{B} A_{i_{n}}^{o}\right)\right)\right)
$$

as a Banach space, where $A_{i_{k}}^{o} \stackrel{\text { def }}{=} A_{i_{k}} \ominus B$, for all $k=1, \ldots, n$. Since our right graph von Neumann algebra $\mathbb{M}_{G}$ is $*$-isomorphic to the $\mathbb{D}_{G}$-free product $*_{\mathbb{D}_{G}} \mathbb{M}_{e}$, $e \in E(G)$

where $\mathbb{M}_{e}$ 's are the $\mathbb{D}_{G}$-free blocks of $\mathbb{M}_{G}$, we can get the Banach space expression. However, the $\mathbb{D}_{G}$-tensor products in our $\mathbb{D}_{G}$-free product is determined by the admissibility on $\mathbb{G}$.

Definition 2.11. Let $G$ be a countable directed graph. Define the subset $E\left(G^{\wedge}\right)_{r}^{*}$ of $F P_{r}\left(G^{\wedge}\right)$ by

$$
E(G)_{r}^{*} \stackrel{\text { def }}{=} E(G) \cup\left(\cup_{k=2}^{\infty}\left\{\begin{array}{l|l}
e_{1} e_{2} \ldots e_{k-1} e_{k} & \begin{array}{c}
e_{1} \ldots e_{k} \in F P_{r}\left(G^{\wedge}\right), \\
e_{1} \neq e_{2}^{ \pm 1}, e_{2} \neq e_{3}^{ \pm 1} \\
\ldots, e_{k-1} \neq e_{k}^{ \pm 1}
\end{array}
\end{array}\right\}\right) .
$$

The following theorem modifies a result from two papers [10] and [11]. In [10], a generalized diagonal $\mathbb{D}_{G}$ is introduced, and associated $\mathbb{D}_{G}$-free blocks in a (left) graph von Neumann algebra are characterized. 
Theorem 2.7. Let $G$ be a countable directed graph and let $\mathbb{M}_{G}=M \times_{\beta} \mathbb{G}$ be the corresponding right graph von Neumann algebra, for a fixed von Neumann algebra $M$. Then, as a Banach space,

$$
\mathbb{M}_{G}=\mathbb{D}_{G} \oplus\left(\underset{w \in E(G)_{r}^{*}}{\oplus} \mathbb{M}_{w}^{o}\right)
$$

Notation To emphasize that $*_{\mathbb{D}_{G}} \mathbb{M}_{e}$ has the above Banach-space expression, $e \in E(G)$

we will denote it by $\underset{\substack{\mathbb{D}_{G} \\ e \in E(G)}}{r} \mathbb{M}_{e}$. And we will call it the $\mathbb{D}_{G}$-valued reduced free product of the $\mathbb{D}_{G}$-free blocks $\mathbb{M}_{e}$ 's.

In [11], the $\mathbb{D}_{G}$-free blocks in a (left) graph von Neumann algebra are characterized. Similarly, we have the following results.

Theorem 2.8. Let $\mathbb{M}_{G}=M \times_{\beta} \mathbb{G}$ be a right graph von Neumann algebra. Then its $\mathbb{D}_{G}$-free block $\mathbb{M}_{e}$ is $*$-isomorphic to either $M \times_{\lambda_{e}} \mathbb{Z}$ (whenever e is a loop-edge) or $M \otimes_{\beta_{e}} M_{2}(\mathbb{C})$ (whenever $e$ is a non-loop edge), where $\lambda_{e}$ is the group action of $\mathbb{Z}$, such that $\lambda_{n}(m)=\beta_{e^{n}}(m)$, for all $m \in M$ and $n \in \mathbb{Z}$.

Assumption In the rest of this paper, we will restrict our interests to the case where $M=\mathbb{C}$.

Suppose $M=\mathbb{C}$. Then a right graph von Neumann algebra $\mathbb{M}_{G}=\mathbb{C} \times{ }_{\beta} \mathbb{G}$ is *-isomorphic to $\overline{\mathbb{C}}[\mathbb{G}]^{w}$, for any graph-representation $\beta$, in $B\left(H_{G}\right)$. Also, in such case, the $\mathbb{D}_{G}$-free blocks are $*$-isomorphic to either $L^{\infty}(\mathbb{T})$ or $M_{2}(\mathbb{C})$.

Example 2.5. Let $G_{N}$ be a one-vertex- $N$-loop-edge graph with its vertex set $V\left(G_{N}\right)$ $=\{v\}$ and $E\left(G_{N}\right)=\left\{e_{j}=v e_{j} v: j=1, \ldots, N\right\}$. Then the right graph von Neumann algebra $\mathbb{C}_{G_{N}}=\mathbb{C} \times{ }_{\beta} \mathbb{G}_{N}$, where $\mathbb{G}_{N}$ is the graph groupoid of $G_{N}$, is *-isomorphic to the free group factor $L\left(F_{N}\right)$ (Also See [10]). Notice that the graph groupoid $\mathbb{G}_{N}$ of $G_{N}$ is a group and moreover it is group-isomorphic to the free group $F_{N}=<g_{1}, \ldots, g_{N}>$ with $N$-generators $\left\{g_{1}, \ldots, g_{N}\right\}$. Indeed, the edges $e_{1}, \ldots, e_{N}$ are generators of $\mathbb{G}_{N}$ as a group. So, there is a natural generator-preserving groupisomorphism between $\mathbb{G}_{N}$ and $F_{N}$. Also, notice that the vertex $v$ in $\mathbb{G}_{N}$ is the group identity. And all partial isometries $R_{e_{j}^{n}}$ correspond to the unitary representation $u_{g_{j}^{n}}$ of $g_{j}^{n}$, for all $n \in \mathbb{Z} \backslash\{0\}$ and for $j=1, \ldots, N$. Therefore, the right graph von Neumann algebra $\mathbb{C}_{G_{N}}=\mathbb{C} \times{ }_{\beta} \mathbb{G}_{N}$ is $*$-isomorphic to the classical crossed product algebra $\mathbb{C} \times{ }_{\lambda} F_{N}$, whenever $\lambda=\beta$. Notice that $\mathbb{C} \times_{\beta} \mathbb{G}_{N}$ is $*$-isomorphic to ${\overline{\mathbb{C}}\left[\mathbb{G}_{N}\right]}^{w}$, for any $\beta$, by the linearity of $\beta$ on $\mathbb{C}$. Also, it is well-known that a crossed product algebra $\mathbb{C} \times_{\gamma} \Gamma$ is *-isomorphic to $\overline{\mathbb{C}}[\Gamma]^{w}$, for any group action $\gamma$, for all groups $\Gamma$. Therefore, the right graph von Neumann algebra $\mathbb{C}_{G_{N}}$ is *isomorphic to the group von Neumann algebra ${\overline{\mathbb{C}}\left[F_{N}\right]}^{w}$. More generally, the right 
graph von Neumann algebra $\mathbb{M}_{G_{N}}=M \times_{\beta} \mathbb{G}_{N}$ is $*$-isomorphic to the classical crossed product von Neumann algebra $M \times_{\lambda} F_{N}$, whenever $\lambda=\beta$.

Notice that if $G$ is a countable directed graph with its graph groupoid $\mathbb{G}$ and if $\mathbb{M}_{G}=\mathbb{C} \times{ }_{\beta} \mathbb{G}$ is a right graph von Neumann algebra induced by $G$ over $\mathbb{C}$, then we can see that $\mathbb{M}_{G}$ is $*$-isomorphic to the von Neumann algebra $\overline{\mathbb{C}[\mathbb{G}]}$, generated by $\mathbb{G}$, by the linearity of $\beta$ on $\mathbb{C}$. Thus, for any graph-representation $\beta$, the corresponding right graph von Neumann algebra $\mathbb{C} \times_{\beta} \mathbb{G}$ is $*$-isomorphic to $\overline{\mathbb{C}}[\mathbb{G}]^{w}$.

Example 2.6. Let $C_{N}$ be the one-flow circulant graph with $V\left(C_{N}\right)=\left\{v_{1}, \ldots, v_{N}\right\}$ and $E\left(C_{N}\right)=\left\{e_{j}=v_{j} e_{j} v_{j+1}: j=1, \ldots, N, v_{N+1} \stackrel{\text { def }}{=} v_{1}\right\}$. Then the right graph von Neumann algebra $\mathbb{C}_{C_{N}}=\mathbb{C} \times{ }_{\beta} \mathbb{G}_{C_{N}}$ contains $W^{*}$-subalgebras which are $*$ isomorphic to the group von Neumann algebra $\overline{\mathbb{C}[\mathbb{Z}]}{ }^{w}$. Since a finite path $w=e_{1}$ $\ldots e_{N}$ induces the subset $\mathbb{S}_{w}$ of $\mathbb{G}_{C_{N}}$ consisting of all reduced words in $S_{w}=\{w$, $\left.w^{-1}\right\}$ in $\mathbb{G}_{C_{N}}$, we have the $W^{*}$-subalgebra $N=\mathbb{C} \times \beta \mathbb{S}_{w}$ in $\mathbb{C}_{C_{N}}$. We can regard this $W^{*}$-subalgebra $N$ as a right graph von Neumann algebra induced by the graph

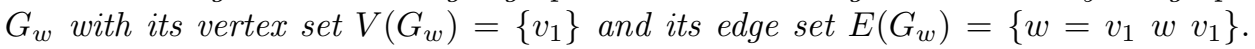
Then it is an one-vertex-one-loop-edge graph. So, by the previous example, the von Neumann algebra $N$ is *-isomorphic to $L\left(F_{1}\right)$, where $F_{1}$ is the free group with 1generator which is group-isomorphic to $\mathbb{Z}$. So, $N$ is $*$-isomorphic to $L(\mathbb{Z})$ which is $*$-isomorphic to $L^{\infty}(\mathbb{T})$, where $\mathbb{T}$ is the unit circle in $\mathbb{C}$. Consider $w^{k}$, for all $k \in \mathbb{Z}$ $\backslash\{0\}$. Then $W^{*}$-subalgebras $N=\mathbb{C} \times{ }_{\beta} \mathbb{G}_{w^{k}}$ of $\mathbb{C}_{C_{N}}$ are all $*$-isomorphic to $L(\mathbb{Z})$.

We can easily check that $E\left(C_{N}\right)_{r}^{*}=F P\left(C_{N}\right) \cup F P\left(C_{N}^{-1}\right)$, for all $N \in \mathbb{N}$. Therefore,

$$
\mathbb{C}_{C_{N}}=\mathbb{C} \times \times_{\beta} \mathbb{G}_{C_{N}}=\underset{\substack{* \mathbb{D}_{C_{N}} \\ k=1}}{N} \mathbb{C}_{e_{k}}
$$

where $\mathbb{C}_{e_{k}} \stackrel{\text { def }}{=} v N\left(\mathbb{C} \times_{\beta} \mathbb{G}_{e}, \mathbb{D}_{C_{N}}\right)$, for all $k=1, \ldots, N$

$$
\begin{aligned}
& =\mathbb{D}_{C_{N}} \oplus\left(\underset{w^{*} \in E\left(C_{N}\right)_{r}^{*}}{\oplus} \mathbb{C}_{w^{*}}^{o}\right) \\
& =\mathbb{D}_{C_{N}} \oplus\left(\underset{w \in F P\left(C_{N}\right) \cup F P\left(C_{N}^{-1}\right)}{\oplus} \mathbb{C}_{w}^{o}\right),
\end{aligned}
$$

where $\mathbb{C}_{w}^{o} \stackrel{\text { def }}{=} \mathbb{C}_{e^{(1)}}^{o} \otimes_{\mathbb{D}_{C_{N}}} \ldots \otimes_{\mathbb{D}_{C_{N}}} \mathbb{C}_{e^{(n)}}^{o}$, whenever $w=e^{(1)} \ldots e^{(n)}$ in $E\left(C_{N}\right)_{r}^{*}$, for $n \in \mathbb{N}$. 


\section{Labeled Graph Groupoids and Graph Automata}

We will give suitable weights to the elements of graph groupoids induced by the given locally finite connected directed graphs. Then these weights give more accurate information of the admissibility on graph groupoids. Such admissibility conditions are explained by the labeling map and the shifting (map) of the automata constructed by graph groupoids. Also, this process would show how the admissibility on a graph groupoid works on the corresponding graph Hilbert space.

Recall that a countable directed graph $G$ is locally finite, if every vertex of $G$ has only finitely many incident edges (equivalently, the degree of each vertex is finite). Also, we say that $G$ is connected, if, for any pair $\left(v_{1}, v_{2}\right)$ of distinct vertices $\left(v_{1} \neq\right.$ $\left.v_{2}\right)$, there always exists at least one reduced finite path $w \in F P_{r}\left(G^{\wedge}\right)$ such that $w$ $=v_{1} w v_{2}$ and $w^{-1}=v_{2} w^{-1} v_{1}$.

Assumption From now on, all given directed graphs are locally finite and connected.

Let $G$ be a locally finite connected directed graph and let $v_{0}$ be an any fixed vertex of $G$. Then we can define the out-degree $\operatorname{deg}_{\text {out }}\left(v_{0}\right)$, the in-degree $\operatorname{deg}_{\text {in }}\left(v_{0}\right)$, and the degree $\operatorname{deg}\left(v_{0}\right)$ of $v_{0}$ as follows:

$$
\begin{aligned}
& \operatorname{deg}_{\text {out }}\left(v_{0}\right) \stackrel{\text { def }}{=}\left|\left\{e \in E(G): e=v_{0} e\right\}\right|, \\
& \operatorname{deg}_{\text {in }}\left(v_{0}\right) \stackrel{\text { def }}{=}\left|\left\{e \in E(G): e=e v_{0}\right\}\right|,
\end{aligned}
$$

and

$$
\operatorname{deg}\left(v_{0}\right) \stackrel{\text { def }}{=} \operatorname{deg}_{\text {out }}\left(v_{0}\right)+\operatorname{deg}_{\text {in }}\left(v_{0}\right) .
$$

By the locally-finiteness of $G$, the above three cardinalities are less than $\infty$. We are interested in out-degrees.

\subsection{Labeled Graph Groupoids.}

As before, let $G$ be a locally finite connected directed graph. By the locally finiteness of $G$, we can have that

$$
\max \left\{\operatorname{deg}_{\text {out }}(v): v \in V(G)\right\}<\infty .
$$

Denote the maximal value of the out-degrees of all vertices by $N$. Then we can define a set

$$
Y=\{1, \ldots, N\} \text { and } Y_{0}=\{0\} \cup Y .
$$


Also, fix sets $X$ and $X_{0}$ of certain vectors in $\mathbb{C}^{\oplus N}$, indexed by $Y$ and $Y_{0}$, respectively:

where

$$
X=\left\{x_{1}, \ldots, x_{N}\right\} \text { and } X_{0}=\left\{x_{0}, x_{1}, \ldots, x_{N}\right\},
$$

$$
x_{0}=(\underbrace{0, \ldots \ldots . ., 0}_{N \text {-times }}) \text { and } x_{j}=(0, \ldots, 0, \underset{j \text {-th }}{1}, 0, \ldots, 0),
$$

for all $j=1, \ldots, N$. Remark that the number $N$ is the maximal out-degree of vertices of $G$.

Definition 3.1. Let $X$ and $X_{0}$ be given as above. We will call both $X$ and $X_{0}=$ $\left\{x_{0}\right\} \cup X$, the (vector) labeling sets of $G\left(\right.$ in $\mathbb{C}^{\oplus N}$ ).

Let's fix $v_{0} \in V(G)$, and let $\operatorname{deg}_{\text {out }}\left(v_{0}\right)=n \leq N$. Define the subset $E_{\text {out }}^{v_{0}}$ of the edge set $E(G)$ by

$$
E_{\text {out }}^{v_{0}} \stackrel{\text { def }}{=}\left\{e \in E(G): e=v_{0} e\right\} \subseteq E(G) .
$$

i.e., $\operatorname{deg}_{\text {out }}(v)=\left|E_{\text {out }}^{v}\right|$, for all $v \in V(G)$. Then we decide the weights $\left\{\left(v_{0}, x_{1}\right)\right.$, $\left.\ldots,\left(v_{0}, x_{n}\right)\right\}$, contained in the set $\left\{v_{0}\right\} \times X$, on $E_{\text {out }}^{v_{0}}$. i.e.,

$$
E_{\text {out }}^{v_{0}}=\left\{\begin{array}{l|l}
e_{\left(v_{0}, x_{1}\right)}, \ldots, e_{\left(v_{0}, x_{n}\right)}: & \begin{array}{c}
\omega_{v_{0}}\left(e_{\left(v_{0}, x_{j}\right)}\right)=\left(v_{0}, x_{j}\right), \\
\forall j=1, \ldots, n
\end{array}
\end{array}\right\},
$$

where

$$
\omega_{v_{0}}: E_{\text {out }}^{v_{0}} \rightarrow\left\{v_{0}\right\} \times\left\{x_{1}, \ldots, x_{n}\right\}
$$

is the weighting process putting the weight $\left(v_{0}, x_{j}\right)$ to an edge $e_{\left(v_{0}, x_{j}\right)}$.

For any $v \in V(G)$, we can do the same process and we have the corresponding weighting process $\omega_{v}$ and the corresponding set

$$
E_{\text {out }}^{v}=\left\{\begin{array}{l|c}
e_{\left(v, x_{1}\right)}, \ldots, e_{\left(v, x_{k}\right)} & \begin{array}{c}
x_{1}, \ldots, x_{k} \in X \\
\text { and } \omega_{v}\left(e_{\left(v, x_{j}\right)}\right)=\left(v, x_{j}\right) \\
\forall j=1, \ldots, k, \text { where } \\
k=\operatorname{deg}_{\text {out }}(v) \leq N
\end{array}
\end{array}\right\} .
$$

Notice that if $\operatorname{deg}_{\text {out }}(v)=0$, then $E_{\text {out }}^{v}$ is empty, by the fact that $\{v\} \times \varnothing=\varnothing$, where $\varnothing$ is the empty set. Notice also that, by the connectedness of $G$,

$$
E(G)=\underset{v \in V(G)}{\sqcup} E_{o u t}^{v}
$$


where "ப" means the disjoint union. So, the each weighting processes $\left(\omega_{v}\right)_{v \in V(G)}$ extends to the weighting process of $E(G)$ by $X$ :

$$
\omega: E(G) \rightarrow V(G) \times X
$$

by

$$
\omega(e)=\omega(v e) \stackrel{\text { def }}{=} \omega_{v}(e)
$$

for all $e=v e \in E(G)$, with $v \in V(G)$.

Now, let $G^{-1}$ be the shadow of $G$. We can determine the similar weighting process on $G^{-1}$. Assume that the graph $G$ is weighted by the weighting process $\omega$ for the labeling set $X$. Define now the sets $-X$ and $-X_{0}$ by

$$
-X \stackrel{\text { def }}{=}\left\{-x_{1}, \ldots,-x_{N}\right\} \text { and }-X_{0}=\left\{x_{0}\right\} \cup(-X),
$$

where

$$
x_{0}=(\underbrace{0, \ldots, 0}_{N \text {-times }}) \text { and }-x_{j}=(0, \ldots, 0, \underset{j \text {-th }}{0,0}, \ldots, 0) \text {, }
$$

for all $j=1, \ldots, N$. i.e., the set $-X$ and $-X_{0}$ are also contained in $\mathbb{C}^{\oplus N}$. Now, consider the set $V\left(G^{-1}\right) \times(-X)=V(G) \times(-X)$. Then, we can get the weighting processes $\left(\omega_{v}^{-1}\right)_{v \in V\left(G^{-1}\right)}$ defined by

such that

$$
\omega_{v}^{-1}: E_{\text {out }}^{v^{-1}} \rightarrow\{v\} \times(-X)
$$

$$
\omega_{v}^{-1}\left(e^{-1}\right)=\omega_{v}^{-1}\left(e^{-1} v\right) \stackrel{\text { def }}{=}\left(v,-x_{i_{j}}\right),
$$

whenever $w_{v}(e)=\left(v, x_{i_{j}}\right) \in\{v\} \times X$. Therefore, we can define the weighting process $\omega^{-1}$ of $E\left(G^{-1}\right)$ by

$$
\omega^{-1}: E\left(G^{-1}\right) \rightarrow V\left(G^{-1}\right) \times(-X)
$$

by

$$
\omega^{-1}\left(e^{-1}\right)=\omega^{-1}\left(e^{-1} v\right) \stackrel{\text { def }}{=}\left(v,-x_{i_{j}}\right),
$$

whenever

$$
\omega(e)=\left(v, x_{i_{j}}\right) \in\{v\} \times X .
$$

Define the canonical projection

$$
p r_{G}: V(G) \times X \rightarrow X
$$

by

$$
\operatorname{pr}_{G}((v, x)) \stackrel{\text { def }}{=} x \text {, for all }(v, x) \in V(G) \times X \text {. }
$$


Similarly, define the canonical projection

by

$$
p r_{G^{-1}}: V(G) \times(-X) \rightarrow-X
$$

$$
\operatorname{pr}_{G^{-1}}((v,-x)) \stackrel{\text { def }}{=}-x \text {, for all }(v,-x) \in V\left(G^{-1}\right) \times(-X) \text {. }
$$

Definition 3.2. Let $G$ be a locally finite connected directed graph equipped with its weighting process $\omega$ of the edge set $E(G)$ by $V(G) \times X$, where $X=\{1, \ldots, N\}$, and $N=\max \left\{\operatorname{deg}_{\text {out }}(v): v \in V(G)\right\}$. Sometimes, we denote the graph $G$ equipped with the weighting process $\omega$ by the pair $(G, \omega)$. Then the graph $(G, \omega)$ is called the canonical weighted graph. Similarly, the weighted graph $\left(G^{-1}, \omega^{-1}\right)$ equipped with the weighting process $\omega^{-1}$ of $E\left(G^{-1}\right)$ by $(-X) \times V(G)$, where $G^{-1}$ is the shadow of $G$, is called the canonical weighted shadow of $(G, \omega)$.

Let $(G, \omega)$ (resp. $\left.\left(G^{-1}, \omega^{-1}\right)\right)$ be the canonical weighted graph (resp. the canonical weighted shadow of $(G, \omega))$. The image $\operatorname{pr}_{G} \circ \omega$ of $E(G)\left(\right.$ resp. $\left.p_{G^{-1}} \circ \omega^{-1}\right)$ contained in $X$ (resp. in $-X)$ is called the canonical labeling process of $(G, \omega)$ (resp. of $\left.\left(G^{-1}, \omega^{-1}\right)\right)$.

Let $(G, \omega)$ be the canonical weighted graph with its canonical weighted shadow $\left(G^{-1}, \omega^{-1}\right)$. If $\mathbb{G}$ is the graph groupoid of $G$, then we can put the canonical weighting process, also denoted by $\omega$, in terms of the canonical weighting process $\omega$ of the free semigroupoid $\mathbb{F}^{+}\left(G^{\wedge}\right)$ of the shadowed graph $G^{\wedge}$ of $G$. Let $X^{*}$ be the collection of all finite words in $X$, containing the empty word $\emptyset_{X}$ in $X$. Similarly, we can determine the set $(-X)^{*}$ consisting of all finite words in $-X$, containing the empty word $\emptyset_{-X}$. Identify $\emptyset_{X}$ and $\emptyset_{-X}$ to $\emptyset_{*}$ in $( \pm X)^{*}$, the collection of all words in $\pm X$ $\stackrel{\text { def }}{=} X \cup(-X)$. i.e.,

$$
( \pm X)^{*} \stackrel{\text { def }}{=}\left\{\emptyset_{*}\right\} \cup\left(\bigcup_{n=1}^{\infty}\left\{\begin{array}{l|l}
x_{i_{1}} \ldots x_{i_{n}} & \begin{array}{c}
x_{i_{1}}, \ldots, x_{i_{n}} \in \pm X, \text { where } \\
\pm X=\left\{ \pm x_{1}, \ldots, \pm x_{N}\right\} \\
\text { with } N=\max _{v \in V(G)} \operatorname{deg}_{\text {out }}(v)
\end{array}
\end{array}\right\}\right.
$$

Also define

Define the map

$$
\pm X_{0}^{*} \stackrel{\text { def }}{=}\left\{x_{0}\right\} \cup( \pm X)^{*} .
$$

$$
\omega: F P\left(G^{\wedge}\right) \cup\{\emptyset\} \rightarrow V(G)^{2} \times\left( \pm X_{0}^{*}\right)
$$

by

$$
\omega(\emptyset) \stackrel{\text { def }}{=} \emptyset_{*} \stackrel{\text { regarded as }}{=}\left(\varnothing, \emptyset_{*}\right)
$$

and

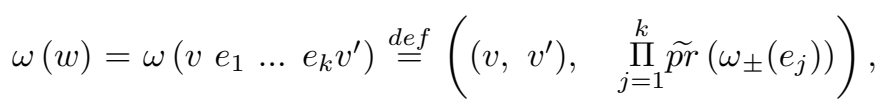


where $\varnothing$ is the empty set in $V(G)^{2}$ and $F P\left(G^{\wedge}\right)$ is the (non-reduced) finite path set of the shadowed graph $\hat{G}$, and where

whenever

$$
\widetilde{p r} \in\left\{p r_{G}, p r_{G^{-1}}\right\} \text { and } \omega_{ \pm} \in\left\{\omega, \omega^{-1}\right\},
$$

$$
w=v w v^{\prime}=e_{1} \ldots e_{k},
$$

with

$$
v, v^{\prime} \in V\left(G^{\wedge}\right), \text { and } e_{1}, \ldots, e_{k} \in E\left(G^{\wedge}\right)
$$

for $k \in \mathbb{N}$. In the above, we have

$$
\omega_{ \pm}: E\left(G^{\wedge}\right)=E(G) \sqcup E\left(G^{-1}\right) \rightarrow \pm X
$$

satisfies

$$
\omega_{ \pm}(e)= \begin{cases}\omega(e) & \text { if } e \in E(G) \\ \omega^{-1}(e) & \text { if } e \in E\left(G^{-1}\right)\end{cases}
$$

and

satisfies

$$
\widetilde{p r}:(V(G) \times X) \cup\left(V\left(G^{-1}\right) \times-X\right) \rightarrow X \cup(-X)
$$

$$
\widetilde{p r}(g)= \begin{cases}p r_{G}(g) & \text { if } g \in V(G) \times X \\ p r_{G^{-1}}(g) & \text { if } g \in V(G) \times(-X) .\end{cases}
$$

The above map $\omega$ from $F P\left(G^{\wedge}\right) \cup\{\emptyset\}$ can be extended to $\mathbb{F}^{+}\left(G^{\wedge}\right)$ into $V(G)^{2}$ $\times\left( \pm X_{0}^{*}\right)$ by putting the weights on vertices:

$$
\omega(v) \stackrel{\text { def }}{=}\left((v, v), x_{0}\right) \text {, for all } v \in V(G) .
$$

Such weighting process of the free semigroupoid $\mathbb{F}^{+}\left(G^{\wedge}\right)$ of $G^{\wedge}$ is called the canonical weighting process of $\mathbb{F}^{+}\left(G^{\wedge}\right)$ or that of $\mathbb{G}$ induced by the canonical weighted $\operatorname{graph}(G, \omega)$.

Notation From now, for convenience, we denote the above weighting process of $\mathbb{F}^{+}\left(G^{\wedge}\right)$ simply by $\omega$. Remark that, set-theoretically, the graph groupoid $\mathbb{G}$ is contained in the free semigroupoid $\mathbb{F}^{+}\left(G^{\wedge}\right)$ of the shadowed graph $G^{\wedge}$ of $G$. So, we can determine the weighting process $\omega$ of $\mathbb{G}$ simply by restricting $\omega$ of $\mathbb{F}^{+}\left(G^{\wedge}\right)$. i.e., the weighting process of $\mathbb{G}$ is $\omega=\left.\omega\right|_{\mathbb{G}}$. We will use the notation $\omega$ for the weighting processes of $\mathbb{F}^{+}\left(G^{\wedge}\right)$ and $\mathbb{G}$, alternatively.

Notice that the above weighting process $\omega$ of $\mathbb{F}^{+}\left(G^{\wedge}\right)$ represents the full admissibility conditions of $\mathbb{G}$. i.e., the weight $\omega(w)$ of $w \in \mathbb{G}$ contains its initial and terminal vertices and the connecting pattern (admissibility of edges) of $w$, in terms of labelings in $\pm X_{0}$.

Definition 3.3. Let $(G, \omega)$ be the canonical weighted graph with its canonical weighted shadow $\left(G^{-1}, \omega^{-1}\right)$. Let $\mathbb{G}$ be the graph groupoid of $G$ and assume that 
$\omega$ is the canonical weighting process of $\mathbb{G}$ induced by $(G, \omega)$. Then we call the pair $(\mathbb{G}, \omega)$ the labeled graph groupoid of $(G, \omega)$. We call the processes $\widetilde{p r} \circ \omega$ on $(\mathbb{G}$, $\omega)$, the labeling process, where $\widetilde{p r} \in\left\{p r_{G}, p r_{G^{-1}}\right\}$, as in the previous paragraph.

Assumption From now, if we mention (locally finite connected) directed graphs, then they are automatically assumed to be the canonical weighted graphs. Similarly, if we mention their graph groupoids, then they are also automatically assumed to be the labeled graph groupoids.

Example 3.1. Let $G$ be the circulant graph with three vertices with

$$
V(G)=\left\{v_{1}, v_{2}, v_{3}\right\}
$$

and

$$
E(G)=\left\{e_{j}=v_{j} e_{j} v_{j+1}: j=1,2,3, \text { with } v_{4} \stackrel{\text { def }}{=} v_{1}\right\}
$$

i.e.,

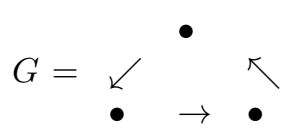

Then, since $\operatorname{deg}_{\text {out }}\left(v_{j}\right)=1$, for all $j=1,2,3$, we can get the labeling sets

$$
X=\left\{x_{1}\right\}=\{1\} \text { and } X_{0}=\left\{x_{0}, x_{1}\right\}=\{0,1\}
$$

contained in $\mathbb{C}^{\oplus 1}=\mathbb{C}$. Relatively, we have that

$$
-X=\left\{-x_{1}\right\}=\{-1\} \text { and }-X_{0}=\left\{-x_{0},-x_{1}\right\}=\{0,-1\},
$$

for the shadow $G^{-1}$ of $G$. Then we can get the weights

$$
\omega\left(e_{j}\right)=\left(\left(v_{j}, v_{j+1}\right), 1\right), \text { for all } j=1,2,3
$$

and

$$
\omega^{-1}\left(e_{j}^{-1}\right)=\left(\left(v_{j+1}, v_{j}\right),-1\right), \text { for all } j=1,2,3,
$$

where $v_{4} \stackrel{\text { def }}{=} v_{1}$, in $V(G)$. We can construct the labeled graph groupoid $(\mathbb{G}, \omega)$. Let $w=e_{2} e_{3} e_{1} \in F P_{r}\left(G^{\prime}\right)$. Then we can get that

$$
\omega(w)=\left(\left(v_{2}, v_{2}\right), 111\right) \text {, and } \omega\left(w^{-1}\right)=\left(\left(v_{2}, v_{2}\right), 111\right) .
$$

Take now the reduced finite path $y=e_{1}^{-1} e_{3}^{-1} \in F P_{r}\left(G^{\wedge}\right)$. Then we can get

$$
\omega(y)=\left(\left(v_{2}, v_{3}\right),(-1)(-1)\right) .
$$

Suppose we have $w^{2}=e_{2} e_{3} e_{1} e_{2} e_{3} e_{1}$ in $F P_{r}\left(G^{\wedge}\right)$. Then the weight of $w^{2}$ is 


$$
\omega\left(w^{2}\right)=\left(\left(v_{2}, v_{2}\right), 111111\right)
$$

Recall that $F P_{r}\left(G^{\wedge}\right)=F P(G) \cup F P\left(G^{-1}\right)$ (See [10] and [11]: Notice that, in general, $\left.F P_{r}\left(G^{\wedge}\right) \supsetneqq F P(G) \cup F P\left(G^{-1}\right)\right)$.

Notation For convenience, we re-define the set $X^{*}$ and $X_{0}^{*}$ of the labeling sets $X$ and $X_{0}$ as follows:

$$
X^{*} \stackrel{\text { def }}{=}\left\{\emptyset_{*}\right\} \cup\left(\bigcup_{k=1}^{\infty}\left\{\left(x_{i_{1}}, \ldots, x_{i_{k}}\right) \in X^{k} \mid x_{i_{1}}, \ldots, x_{i_{n}} \in X\right\}\right)
$$

and

$$
X_{0}^{*} \stackrel{\text { def }}{=}\left\{x_{0}\right\} \cup X^{*}
$$

i.e., instead of writing the elements of $X^{*}$ as finite words in $X$, we will write them as a finite tuples having their entries in $X$.

\subsection{The Operation $\theta$ and $\omega_{+}$.}

In this section, we will define a certain operation on the labeling set $\pm X_{0}^{*}$ of the labeled graph groupoid $(\mathbb{G}, \omega)$ induced by the canonical weighted graph $(G, \omega)$. Notice that the set $\pm X_{0}^{*}$ is a subset of the $\mathbb{C}$-vector space $\mathbb{C}^{\oplus \infty}$. Let

$$
N \stackrel{\text { def }}{=} \max \left\{\operatorname{deg}_{\text {out }}(v): v \in V(G)\right\} \in \mathbb{N} \text {. }
$$

By regarding all elements $\pm X_{0}^{*} \subset \mathbb{C}^{\oplus \infty}$, as finite tuples of their entries in $\mathbb{C}^{\oplus N}$, we can define an operation $\theta$ from $\pm X_{0}^{*}$ into $\mathbb{C}^{\oplus N}$ by

$$
\theta\left(\left(t_{i_{1}}, \ldots, t_{i_{n}}\right)\right) \stackrel{\text { def }}{=} \sum_{j=1}^{n} t_{i_{j}} \in \mathbb{C}^{\oplus N},
$$

for all $\left(t_{i_{1}}, \ldots, t_{i_{n}}\right) \in \pm X_{0}^{*}$, for $n \in \mathbb{N}$, where

$$
t_{i_{j}} \stackrel{\text { def }}{=} \begin{cases}x_{i_{j}} & \text { if } t_{i_{j}} \in X_{0} \\ -x_{i_{j}} & \text { if } t_{i_{j}} \in-X .\end{cases}
$$

By help of $\theta$, we can define the operation $\omega_{+}$by

$$
\omega_{+}: V(G)^{2} \times\left( \pm X_{0}^{*}\right) \rightarrow V(G)^{2} \times \mathbb{C}^{\oplus N}
$$

by 


$$
\begin{aligned}
\omega_{+}\left(\left(v, v^{\prime}\right),\left(t_{i_{1}}, \ldots, t_{i_{n}}\right)\right) & \stackrel{\text { def }}{=}\left(\left(v, v^{\prime}\right), \theta\left(\left(t_{i_{1}}, \ldots, t_{i_{n}}\right)\right)\right) \\
& =\left(\left(v, v^{\prime}\right), \sum_{j=1}^{n} t_{i_{j}}\right),
\end{aligned}
$$

for all $\left(\left(v, v^{\prime}\right),\left(t_{i_{1}}, \ldots, t_{i_{n}}\right)\right) \in V(G)^{2} \times\left( \pm X_{0}^{*}\right)$. Later, we will use the above operation $\omega_{+}$to verify the amalgamated moments of the labeling operators on right graph von Neumann algebras.

The operation $\theta$ provides the way to detect the reduction $(\mathrm{RR})$ on $\mathbb{G}$. Suppose we have two reduced finite path $w_{1}$ and $w_{2}$ having

$$
\omega\left(w_{1}\right)=\left(\left(v, v^{\prime}\right), X_{1}\right) \text { and } \omega\left(w_{2}\right)=\left(\left(v^{\prime}, v\right), X_{2}\right)
$$

where $X_{1}, X_{2} \in \pm X_{0}^{*} \backslash\left\{\emptyset_{*}\right\}$. Since $w_{1}=w_{1} v^{\prime}$ and $w_{2}=v^{\prime} w_{2}$, we can realize that $w_{1}$ and $w_{2}$ are admissible. i.e., $w_{1} w_{2} \neq \emptyset$ in $\mathbb{G}$. However, we do not know $w_{1}$ $w_{2}$ is either a vertex $v$ or a loop finite path $v\left(w_{1} w_{2}\right) v$ in $\mathbb{G}$. Here, the operation $\theta$ (and hence the operation $\omega_{+}$) provides the way to detect $w_{1} w_{2}$ is either the vertex $v$ or the loop finite path $w_{1} w_{2}$ in $\mathbb{G}$. In conclusion, we can get that:

Proposition 3.1. Let $w_{1}$ and $w_{2}$ be the reduced finite paths in $F P_{r}\left(G^{\wedge}\right)$ given in the previous paragraph. Then

$$
\theta\left((\tilde{p r} \circ \omega)\left(w_{1} w_{2}\right)\right)=x_{0} \text { in } \mathbb{C}^{\oplus N}
$$

or equivalently,

$$
\omega_{+}\left(\omega\left(w_{1} w_{2}\right)\right)=\left((v, v), x_{0}\right)
$$

if and only if the element $w_{1} w_{2} \in \mathbb{G} \backslash\{\emptyset\}$ is identical to the vertex $v$.

Proof. $(\Rightarrow)$ Suppose $\omega_{+}\left(\omega\left(w_{1} w_{2}\right)\right)=\left((v, v), x_{0}\right)$. By hypothesis, we have that

$$
\omega\left(w_{1} w_{2}\right)=\left((v, v),\left(t_{i_{1}}, \ldots, t_{i_{n}}, t_{j_{1}}, \ldots, t_{j_{k}}\right)\right)
$$

whenever

$$
\omega\left(w_{1}\right)=\left(\left(v, v^{\prime}\right),\left(t_{i_{1}}, \ldots, t_{i_{n}}\right)\right)
$$

and

$$
\omega\left(w_{2}\right)=\left(\left(v^{\prime}, v\right),\left(t_{j_{1}}, \ldots, t_{j_{k}}\right)\right)
$$

By assumption, $\theta\left(\left(t_{i_{1}}, \ldots, t_{i_{n}}, t_{j_{1}}, \ldots, t_{j_{k}}\right)\right)=x_{0}$. And since $w_{1}$ and $w_{2}$ are "reduced" finite paths in $\mathbb{G}$, we can verify that (i) $n=k$, and (ii) for any $t_{i_{p}}$, there should be the unique entry $t_{j_{q}}=-t_{i_{p}}$, for $p, q=1, \ldots, n=k$. This shows that, if $w_{1}=e_{i_{1}} \ldots e_{i_{n}}$ and $w_{2}=e_{j_{1}} \ldots e_{j_{n}}$ in $F P_{r}\left(G^{\wedge}\right)$, with $e_{i_{1}}, \ldots, e_{i_{n}}, e_{j_{1}}, \ldots, e_{j_{n}} \in$ $E(\hat{G})$, then $e_{j_{q}}=e_{i_{p}}^{-1}$, uniquely, for $p, q=1, \ldots, n$. Therefore, $w_{1} w_{2}=v$. 


$$
\begin{aligned}
& (\Leftarrow) \text { Suppose } w_{1} w_{2}=v \in V(G) \text {. Then } \\
& \qquad \begin{array}{l}
\omega_{+}\left(\omega\left(w_{1} w_{2}\right)\right)=\omega_{+}(\omega(v))=\omega_{+}\left((v, v), x_{0}\right)=\left((v, v), x_{0}\right) .
\end{array}
\end{aligned}
$$

The above proposition shows how the labeling (or weighting) works on the graph groupoid $\mathbb{G}$. The labeling process on $\mathbb{G}$ gives a way to check how admissibility works under $(\mathrm{RR})$.

\subsection{Graph Automata.}

In this section, we will construct an automaton induced by the labeling graph groupoid $\mathbb{G}=(\mathbb{G}, \omega)$ of the canonical weighted graph $G=(G, \omega)$. Let $X_{0}=\left\{x_{0}\right.$, $\left.x_{1}, \ldots, x_{N}\right\}$ be the labeling set of the countable locally finite connected directed graph $G$ contained in $\mathbb{C}^{\oplus N}$, where

$$
N \stackrel{\text { def }}{=} \max \left\{\operatorname{deg}_{\text {out }}(v): v \in V(G)\right\} \text {. }
$$

Then we can create the automaton $\mathcal{A}_{G}=\left\langle\mathcal{X}_{0}, \mathbb{F}^{+}\left(G^{\wedge}\right), \varphi, \psi\right\rangle$, where $G^{\wedge}=$ $G \cup G^{-1}$ is the shadowed graph of the given canonical weighted graph $G$ and

$$
\mathcal{X}_{0} \stackrel{\text { def }}{=}\left\{\emptyset_{G}\right\} \cup\left(V(G)^{2} \times\left( \pm X_{0}\right)\right)
$$

where

$$
\pm X_{0}=X_{0} \cup(-X) \subset \mathbb{C}^{\oplus N}
$$

and

$\varphi\left(\left(\left(v_{1}, v_{2}\right), t\right), e\right) \stackrel{\text { def }}{=}\left\{\begin{array}{cc} & \text { if } e=v_{2} e \text { in } E\left(G^{\prime}\right), \text { equivalently, } \\ \omega(e) \quad & \exists v^{\prime} \in V(G) \text { and } t^{\prime} \in \pm X_{0} \text { s.t. }, \\ & \omega(e)=\left(\left(v_{2}, v^{\prime}\right), t^{\prime}\right) \neq \emptyset_{*} \\ \emptyset_{G} & \text { otherwise, }\end{array}\right.$

and

$$
\psi\left(\left(\left(v_{1}, v_{2}\right), t\right), e\right) \stackrel{\text { def }}{=} \begin{cases}e & \text { if } e=v_{2} e \text { in } E\left(G^{\wedge}\right) \\ \emptyset & \text { otherwise }\end{cases}
$$

where $\emptyset_{G}$ is the empty element of $\mathcal{X}_{0}$ and $\emptyset$ is the empty element in $\mathbb{G}$.

Definition 3.4. We will say that the automaton $\mathcal{A}_{G}=\left\langle\mathcal{X}_{0}, \mathbb{F}^{+}\left(G^{\wedge}\right), \varphi, \psi\right\rangle$ is the graph automaton induced by the canonical weighted graph $G=(G, \omega)$. Sometimes, we call $\varphi$ and $\psi$, the labeling map and the shift (or shifting map), respectively. 
We can realize that the graph automaton $\mathcal{A}_{G}$ induced by $G$ is identically same with the automaton $\mathcal{A}_{G^{-1}}$ induced by the shadow $G^{-1}$ of $G$. Now, fix an edge $e_{0}$ $\in E\left(G^{\wedge}\right)$ and a reduced finite path $w=v_{1} e_{1} \ldots e_{n} v_{n}^{\prime} \in F P_{r}\left(G^{\wedge}\right)$, with $e_{j}=v_{j} e_{j}$ $v_{j}^{\prime} \in E\left(G^{\wedge}\right)$, and $v_{j}, v_{j}^{\prime} \in V\left(G^{\wedge}\right)$, for all $j=1, \ldots, n$, for some $n \in \mathbb{N}$. Then we can define $\varphi$ and $\psi$ inductively on the free semigroupoid $\mathbb{F}^{+}\left(G^{\wedge}\right)$ of $G^{\wedge}$ as follows:

$$
\begin{aligned}
\varphi\left(\left(\left(v_{1}, v_{n}^{\prime}\right),\left(\omega\left(e_{1}\right), \ldots, \omega\left(e_{n}\right)\right)\right), e_{0}\right) \\
=\varphi\left(\omega(w), e_{0}\right) \\
\stackrel{\text { def }}{=} \begin{cases}\omega\left(e_{0}\right) & \text { if } w e_{0} \neq \emptyset \\
\emptyset_{G} & \text { otherwise }\end{cases}
\end{aligned}
$$

and similarly,

$$
\begin{aligned}
\psi\left(\left(\left(v_{1}, v_{n}^{\prime}\right),\left(\omega\left(e_{1}\right), \ldots, \omega\left(e_{n}\right)\right)\right), e_{0}\right) \\
=\psi\left(\omega(w), e_{0}\right) \\
\stackrel{\text { def }}{=} \begin{cases}e_{0} & \text { if } w e_{0} \neq \emptyset \\
\emptyset & \text { otherwise }\end{cases}
\end{aligned}
$$

for $n \in \mathbb{N}$. Also, inductively, we have that, if $w^{\prime}=e_{1}^{\prime} \ldots e_{m}^{\prime} \in F P_{r}\left(G^{\wedge}\right)$, for $m$ $\in \mathbb{N}$, then

$$
\begin{gathered}
\varphi\left(\left(\left(v_{1}, v_{n}^{\prime}\right),\left(\omega\left(e_{1}\right), \ldots, \omega\left(e_{n}\right)\right)\right), w^{\prime}\right) \\
\stackrel{\text { def }}{=} \begin{cases}\omega\left(e_{m}^{\prime}\right) & \text { if } w w^{\prime} \neq \emptyset \\
\emptyset_{G} & \text { otherwise }\end{cases}
\end{gathered}
$$

and similarly,

$$
\begin{gathered}
\psi\left(\left(\left(v_{1}, v_{n}^{\prime}\right),\left(\omega\left(e_{1}\right), \ldots, \omega\left(e_{n}\right)\right)\right), w^{\prime}\right) \\
\stackrel{\text { def }}{=} \begin{cases}w^{\prime} & \text { if } w w^{\prime} \neq \emptyset \\
\emptyset & \text { otherwise }\end{cases}
\end{gathered}
$$

Then, we can construct the automata actions $\left\{\mathcal{A}_{e}: e \in E\left(G^{\wedge}\right)\right\}$ of $\mathcal{A}_{G}$, acting on the set $V(G)^{2} \times\left( \pm X_{0}^{*}\right)$ (consisting of all finite words in $\left.\mathcal{X}_{0}\right)$. Indeed, we can define the action $\mathcal{A}_{e}$ by

$$
\mathcal{A}_{e}\left(\left(\left(v_{1}, v_{2}\right),\left(x_{i_{1}}, \ldots, x_{i_{n}}\right)\right)\right) \stackrel{\text { def }}{=} \varphi\left(\left(\left(v_{1}, v_{2}\right),\left(x_{i_{1}}, \ldots, x_{i_{n}}\right)\right), e\right)
$$

for all $\left(\left(v_{1}, v_{2}\right),\left(x_{i_{1}}, \ldots, x_{i_{n}}\right)\right) \in V(G)^{2} \times\left( \pm X_{0}^{*}\right)$, for all $e \in E\left(G^{\wedge}\right)$. It is easy to be checked that the actions $\mathcal{A}_{e}$ 's satisfies 


$$
\mathcal{A}_{e_{1}} \mathcal{A}_{e_{2}}=\mathcal{A}_{e_{2} e_{1}}, \text { for all } e_{1}, e_{2} \in E\left(G^{\wedge}\right) \text {. }
$$

Thus the actions $\left\{\mathcal{A}_{v}: v \in V\left(G^{\wedge}\right)\right\}$ are induced by $\mathcal{A}_{e}$ 's since

$$
\mathcal{A}_{v} \stackrel{\text { def }}{=} \mathcal{A}_{e e^{-1}}=\mathcal{A}_{e^{-1}} \mathcal{A}_{e} \text {, whenever } e=v e \in E\left(G^{\wedge}\right)
$$

This guarantees that the automata actions $\left\{\mathcal{A}_{e}: e \in E\left(G^{\wedge}\right)\right\}$ on $V(G)^{2} \times \pm X_{0}^{*}$ generate the groupoid which is groupoid-isomorphic to the graph groupoid $\mathbb{G}$ of $G$. i.e., we can have that:

Theorem 3.2. The set $\left\{\mathcal{A}_{e}: e \in E\left(G^{\wedge}\right)\right\}$ of automata actions generates the actions $\left\{\mathcal{A}_{w}: w \in \mathbb{F}^{+}\left(G^{\wedge}\right)\right\}$ of $\mathcal{A}_{G}$. And the groupoid generated by this set $\left\{\mathcal{A}_{e}: e \in E\left(G^{\wedge}\right)\right\}$ is groupoid-isomorphic to the graph groupoid $\mathbb{G}$ of $G$.

\section{Fractaloids}

As we assumed, all graphs in this Section are locally finite connected countable directed graphs and they are canonically weighted by the labeling set. So, the corresponding graph groupoids are labeled.

\subsection{Fractaloids.}

As before, the standing assumption for our graphs $G$ are as follows: locally finite, connected, and countable. The edges of $G$ are directed, and they are assigned weights with the use of a labeling set. In this Section, we will consider a certain special labeled graph groupoids. Let $\mathbb{G}$ be the labeled graph groupoid induced by a locally finite directed graph $G$, with the labeling set $\pm X_{0}=\left\{x_{0}, \pm x_{1}, \ldots, \pm x_{N}\right\}$, and let the canonical weighting process $\omega$ of the free semigroupoid $\mathbb{F}^{+}\left(G^{\wedge}\right)$ of the shadowed graph $G^{\wedge}$ of $G$, be given, where

$$
N \stackrel{\text { def }}{=} \max \left\{\operatorname{deg}_{\text {out }}(v): v \in V(G)\right\}
$$

We observed that the canonical weighted graph $G$ creates the corresponding graph automaton

where

$$
\mathcal{A}_{G}=<\mathcal{X}_{0}, \mathbb{F}^{+}\left(G^{\wedge}\right), \varphi, \psi>
$$




$$
\mathcal{X}_{0}=\left\{\emptyset_{*}\right\} \cup\left(V(G)^{2} \times\left( \pm X_{0}\right)\right),
$$

and $\varphi$ is the labeling map and $\psi$ is the shifting map. We already observed that $\mathbb{G}$ acts on a $\mathbb{F}^{+}\left(G^{*}\right)$-set $\mathcal{X}_{0}^{*}$, where $\mathcal{X}_{0}^{*}$ is the collection of all finite (non-reduced) words in $\mathcal{X}_{0}$.

Recall that two countable directed graphs $G_{1}$ and $G_{2}$ are graph-isomorphic, if there exists a bijection

$$
g: V\left(G_{1}\right) \cup E\left(G_{1}\right) \rightarrow V\left(G_{2}\right) \cup E\left(G_{2}\right),
$$

such that (i) $g\left(V\left(G_{1}\right)\right)=V\left(G_{2}\right)$ and $g\left(E\left(G_{1}\right)\right)=E\left(G_{2}\right)$, and (ii) $g(e)=g\left(v_{1}\right.$ e $\left.v_{2}\right)=g\left(v_{1}\right) g(e) g\left(v_{2}\right)$ in $E\left(G_{2}\right)$, whenever $e=v_{1}$ e $v_{2} \in E\left(G_{1}\right)$, with $v_{1}, v_{2} \in$ $V\left(G_{1}\right)$.

In [10] and [11], we showed that if two graphs $G_{1}$ and $G_{2}$ are graph-isomorphic, then the corresponding graph grouopoids $\mathbb{G}_{1}$ and $\mathbb{G}_{1}$ are groupoid-isomorphic. More generally, if two graphs $G_{1}$ and $G_{2}$ have the graph-isomorphic shadowed graphs, then the graph groupoids $\mathbb{G}_{1}$ and $\mathbb{G}_{1}$ are groupoid-isomorphic. Also, we showed that if two graph groupoids $\mathbb{G}_{1}$ and $\mathbb{G}_{2}$ are groupoid-isomorphic, then the (left) graph von Neumann algebras ${\overline{\mathbb{C}}\left[\mathbb{G}_{1}\right]}^{w}$ and ${\overline{\mathbb{C}}\left[\mathbb{G}_{2}\right]}^{w}$ are $*$-isomorphic, as $W^{*}$-subalgebras in the operator algebra $B\left(H_{G_{1}}\right) \stackrel{\text { identically same }}{=} B\left(H_{G_{2}}\right)$. Therefore, if two graphs $G_{1}$ and $G_{2}$ have the graph-isomorphic shadowed graphs, then the right graph von Neumann algebras $\mathbb{M}_{G_{1}}$ and $\mathbb{M}_{G_{2}}$ are $*$-isomorphic, too.

Recall also that we say a directed graph $G$ is a (directed) tree if this graph $G$ is connected and it has no loop finite paths in $\mathbb{F}^{+}(G)$. Also, we say that a directed tree is rooted, if we can find-and-fix a vertex $v_{0}$ of $G$, with $\operatorname{deg}_{i n}\left(v_{0}\right)=0$. This fixed vertex $v_{0}$ is called the root of $G$. For instance, a graph

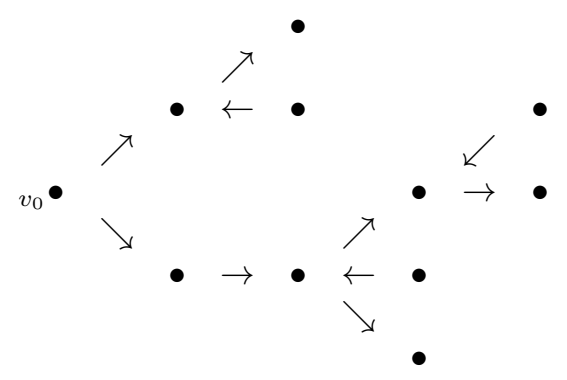

is a rooted tree with its (fixed) root $v_{0}$. A rooted tree $G$ is one-flow, if the directions of edges oriented only one way from the root $v_{0}$. For example, a graph 


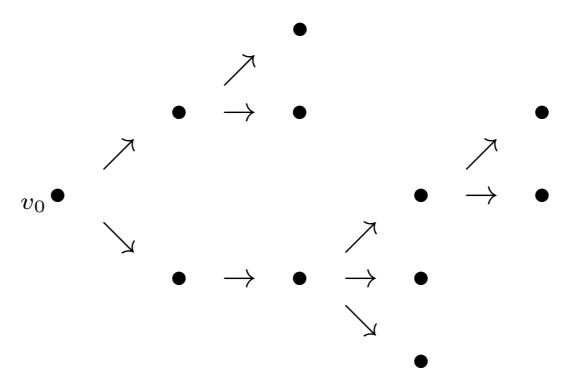

is a one-flow rooted tree with its root $v_{0}$. A one-flow rooted tree $G$ is said to be growing if $G$ is an infinitely countable directed graph. Finally, we will say that a one-flow growing rooted tree $G$ is regular if the out-degrees of all vertices are identical. For example, a graph

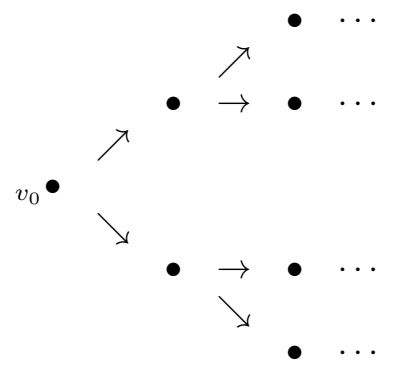

is a regular one-flow growing rooted tree. In particular, if $\operatorname{deg}_{\text {out }}(v)=N$, for all vertices $v$, then this regular one-flow growing rooted tree is called the $N$-regular tree. The very above example is the 2-regular tree.

Let $N$ be the maximal out-degree of the graph $G$, and let $\mathcal{T}_{2 N}$ be the $2 N$-regular tree. Then the automata actions $\left\{\mathcal{A}_{w}: w \in \mathbb{F}^{+}\left(G^{\wedge}\right)\right\}$ of the graph automaton $\mathcal{A}_{G}$ acts on this $2 N$-regular tree $\mathcal{T}_{2 N}$. For instance, for the set $\left\{\mathcal{A}_{w}\right\}_{w \in \mathbb{F}^{+}\left(G^{\wedge}\right)}$ of automata actions of the graph automaton $\mathcal{A}_{G}$, we can create a one-flow growing rooted tree $\mathcal{T}_{G}$ having its arbitrarily fixed root $\omega\left(v_{0}\right)=\left(\left(v_{0}, v_{0}\right), x_{0}\right) \in V(G)^{2} \times$ $\left( \pm X_{0}^{*}\right)$, where

$$
V\left(\mathcal{T}_{G}\right) \stackrel{\text { def }}{=}\left\{\begin{array}{l|l}
\varphi(X, e) & \begin{array}{c}
X \in V(G)^{2} \times\left( \pm X_{0}^{*}\right), \\
e \in E\left(G^{*}\right)
\end{array}
\end{array}\right\}
$$

and

$$
E\left(\mathcal{T}_{G}\right) \stackrel{\text { def }}{=}\left\{\psi(X, e) \mid \begin{array}{c}
X \in V(G)^{2} \times\left( \pm X_{0}^{*}\right) \\
e \in E\left(G^{*}\right)
\end{array}\right\},
$$

where $G^{\wedge}$ is the shadowed graph of $G$.

Observation and Notation Notice that, by the connectedness of the shadowed graph $G^{\wedge}$ of the connected graph $G$, we can fix any weight $\omega(v)$ of $v \in V\left(G^{\wedge}\right)$, as 
the root of the tree $\mathcal{T}_{G}$. Suppose $\mathcal{T}_{v_{1}}$ and $\mathcal{T}_{v_{2}}$ are the one-flow growing trees with their roots $\left(\left(v_{1}, v_{1}\right), x_{0}\right)$ and $\left(\left(v_{2}, v_{2}\right), x_{0}\right)$, respectively. Then, $\mathcal{T}_{v_{i}}$ is embedded in $\mathcal{T}_{v_{j}}$, as a full-subgraph (See the definition of full-subgraphs below), whenever $i \neq j$ $\in\{1,2\}$. In general, the graphs $\mathcal{T}_{v_{1}}$ and $\mathcal{T}_{v_{2}}$ have no graph-isomorphic relation, but they are embedded from each other, by the connectedness of $G$. Since $v_{1}$ and $v_{2}$ are arbitrary, we can consider only one choice $\mathcal{T}_{v}$, for $v \in V(G)$, as a candidate of the tree, denoted by $\mathcal{T}_{G}$, as the tree where the automata actions act. i.e., whenever we choose one tree $\mathcal{T}_{v}$, for $v \in V(G)$, then the trees $\mathcal{T}_{v^{\prime}}$ 's are embedded in $\mathcal{T}_{v}$, for all $v^{\prime} \in V(G)$. Without loss of generality, if we write $\mathcal{T}_{G}$ from now, then it means a tree $\mathcal{T}_{v}$, for a fixed $v \in V(G)$. Remark that, the tree $\mathcal{T}_{G}$ has its root $\omega(v)$ if and only if $\mathcal{T}_{G}=\mathcal{T}_{v}$.

We can easily check that

and hence

$$
F P\left(\mathcal{T}_{G}\right)=\left\{\psi(X, w) \mid \begin{array}{c}
X \in V(G)^{2} \times\left( \pm X_{0}^{*}\right), \\
w \in F P\left(G^{*}\right)
\end{array}\right\},
$$

$$
\begin{aligned}
\mathbb{F}^{+}\left(\mathcal{T}_{G}\right)=\left\{\emptyset_{*}\right\} \cup\left\{\varphi(X, e) \mid \begin{array}{c}
X \in V(G)^{2} \times\left( \pm X_{0}^{*}\right), \\
e \in E\left(G^{\wedge}\right)
\end{array}\right\} \\
\cup\left\{\psi(X, w) \mid \begin{array}{c}
X \in V(G)^{2} \times\left( \pm X_{0}^{*}\right), \\
w \in F P\left(G^{\wedge}\right)
\end{array}\right\} .
\end{aligned}
$$

By the connectedness of the graph $G$, and by the definition of the automata actions, every finite paths in $F P\left(G^{\wedge}\right)$ is embedded in the finite paths of the tree $\mathcal{T}_{G}$, via the automata actions. Then, we can construct the full-subgraphs $\left\{\mathcal{T}_{w}: w\right.$ $\left.\in F P\left(G^{\wedge}\right)\right\}$ of $\mathcal{T}_{G}$, where $\mathcal{T}_{w}$ 's are the one-flow growing rooted tree with their roots $\omega(w)$, for all $w \in F P\left(G^{\wedge}\right)$.

Recall that, we say that a countable directed graph $G_{1}$ is a full-subgraph of a countable directed graph $G_{2}$, if

$$
E\left(G_{1}\right) \subseteq E\left(G_{2}\right)
$$

and

$$
V\left(G_{1}\right)=\left\{\begin{array}{l|l}
v \in V\left(G_{2}\right) & \begin{array}{c}
e=v e \text { or } e=e v \\
\forall e \in E\left(G_{1}\right)
\end{array}
\end{array}\right\}
$$

Notice the difference between full-subgraphs and subgraphs. We say that $G_{1}$ is a subgraph of $G_{2}$, if

$$
V\left(G_{1}\right) \subseteq V\left(G_{2}\right)
$$

and

$$
E\left(G_{1}\right)=\left\{\begin{array}{l|l}
e \in E\left(G_{2}\right) & \begin{array}{c}
e=v e v^{\prime} \\
\forall v, v^{\prime} \in V\left(G_{2}\right)
\end{array}
\end{array}\right\} .
$$

Every subgraph is a full-subgraph, but the converse does not hold true. 
Definition 4.1. Let $\mathcal{T}_{G}$ be the above one-flow growing rooted tree, where the automata actions of the graph automaton $\mathcal{A}_{G}$ act. We say that the tree $\mathcal{T}_{G}$ is the $\mathcal{A}_{G^{-}}$ tree, which is a full-subgraph of the $2 N$-regular tree $\mathcal{T}_{2 N}$. The full-subgraphs $\mathcal{T}_{w}$ 's for $w \in F P\left(G^{\wedge}\right)$ of $\mathcal{T}_{G}$ are called the w-parts of $\mathcal{T}_{G}$, which are also full-subgraphs of $\mathcal{T}_{2 N}$.

The important thing is now that the $w$-parts $\mathcal{T}_{w}$ 's are embedded in the $2 \mathrm{~N}$ regular tree $\mathcal{T}_{2 N}$, and $\mathcal{T}_{w}$ 's are embedded in $\mathcal{T}_{w}$ 's, whenever

where

$$
\varphi\left(\omega(v), w^{\prime}\right)=\left(\left(v, v^{\prime}\right), X^{\prime}\right)
$$

$$
\varphi(\omega(v), w)=\left(\left(v, v^{\prime \prime}\right), X\right) \text { and } X^{\prime}=\left(X, X^{\prime \prime}\right),
$$

for some $X^{\prime \prime} \in\left( \pm X_{0}^{*}\right)$, where $\omega(v)$ is the root of $\mathcal{T}_{G}$.

Remark 4.1. The construction of the $\mathcal{A}_{G}$-tree $\mathcal{T}_{G}$ is nothing but the rearrangement of the finite paths in the free semigroupoid $\mathbb{F}^{+}\left(G^{\wedge}\right)$ of the shadowed graph $G^{\wedge}$ inside the $2 N$-regular tree $\mathcal{T}_{2 N}$, up to the admissibility on $\mathbb{F}^{+}\left(G^{\wedge}\right)$. Notice that, in fact, the $\mathcal{A}_{G}$-tree $\mathcal{T}_{G}$ contains the information about the vertices in $\mathbb{F}^{+}\left(G^{\wedge}\right)$, too, since the vertices of $\mathcal{T}_{G}$ are contained in $V(G)^{2} \times\left( \pm X_{0}^{*}\right)$. Remark that, by the connectedness of $G^{\wedge}$, the w-parts $\mathcal{T}_{w}$ 's of $\mathcal{T}_{G}$ for $w \in F P\left(G^{\wedge}\right)$ are well-constructed as a one-flow growing tree with their roots $\varphi(\omega(v), w)$, where $\omega(v)$ is the root of $\mathcal{T}_{G}$. Moreover, each tree $\mathcal{T}_{w}$ is embedded in the other trees $\mathcal{T}_{G}$.

Notice that, even though $|\mathbb{G}|<\infty,\left|\mathbb{F}^{+}\left(G^{\wedge}\right)\right|=\infty$, whenever $|E(G)| \geq 1$. By identifying their roots, the $\mathcal{A}_{G}$-tree $\mathcal{T}_{G}$ is a full-subgraph of the $2 N$-regular tree $\mathcal{T}_{2 N}$. Therefore, we can conclude that:

Lemma 4.1. The automata actions $\left\{\mathcal{A}_{w}: w \in \mathbb{F}^{+}\left(G^{\wedge}\right)\right\}$ of the graph automaton $\mathcal{A}_{G}$, induced by the given graph $G$, act on the $2 N$-regular tree $\mathcal{T}_{2 N}$.

Proof. By definition, the automata actions $\left\{\mathcal{A}_{w}\right\}_{w \in \mathbb{F}^{+}\left(G^{\wedge}\right)}$ act on the $\mathcal{A}_{G^{-}}$-tree $\mathcal{T}_{G}$. And the tree $\mathcal{T}_{G}$ is a full-subgraph of $\mathcal{T}_{2 N}$.

Let $\mathcal{A}_{G}=<\mathcal{X}_{0}, \mathbb{F}^{+}\left(G^{\wedge}\right), \varphi, \psi>$ be the graph automaton induced by the canonical weighted graph $G=(G, \omega)$. In Section 3.3, we showed that the groupoid $\mathbb{G}\left(\mathcal{A}_{G}\right)$ generated by the automata actions $\left\{\mathcal{A}_{w}: w \in \mathbb{F}^{+}\left(G^{\wedge}\right)\right\}$ is groupoid-isomorphic to the graph groupoid $\mathbb{G}$ of $G$. This is the graph-groupoid version of the fact that: if $\mathcal{A}=\left\langle X^{ \pm}, X^{ \pm *}, \varphi, \psi\right\rangle$ is an automaton, where $X$ is the generator set, with $X^{ \pm}$ $=\left\{x^{ \pm 1}: x \in X\right\}$, of a group $\Gamma$ and $X^{*}$ is the free monoid of the group $\Gamma$, which is monoid-homomorphic to $\Gamma$, then the automata actions $\left\{\mathcal{A}_{y}: y \in X^{ \pm *}\right\}$ generate the automata group $\Gamma(\mathcal{A})$, which is group-isomorphic to $\Gamma$. 
In fact, this fact is not so interesting in Automata Theory and Groupoid Theory, however, for our works, it plays a key role, since we can consider the labeled graph groupoid $\mathbb{G}$ and the automata groupoid $\mathbb{G}\left(\mathcal{A}_{G}\right)$, alternatively, as groupoidisomorphic objects. Also, it provides the technique to see the (reduced or nonreduced) finite paths of the shadowed graph $G^{\wedge}$ as those of $\mathcal{T}_{2 N}$ (as embedded elements).

Definition 4.2. Let $G, \mathbb{F}^{+}\left(G^{\wedge}\right)$, and $\mathbb{G}$ be given as before and let $\mathcal{A}_{G}=<\mathcal{X}_{0}$, $\mathbb{F}^{+}\left(G^{\wedge}\right), \varphi, \psi>$ be the graph automaton induced by $G$, acting on the $2 N$-regular tree $\mathcal{T}_{2 N}$. For any fixed $w \in F P\left(G^{\wedge}\right)$, the tree $\mathcal{T}_{w}$ is the $w$-part of the $\mathcal{A}_{G}$-tree $\mathcal{T}_{G}$, with its root $\varphi(\omega(v), w)$, where $\omega(v)$ is the root of the $\mathcal{A}_{G}$-tree $\mathcal{T}_{G}$. Let $\mathbb{G}\left(\mathcal{T}_{w}\right)$ be the groupoid generated by the actions $\mathcal{A}_{y}$ 's acting only on $\mathcal{T}_{w}$. If $\mathbb{G}\left(\mathcal{T}_{w}\right)$ 's are groupoid-isomorphic to $\mathbb{G}\left(\mathcal{T}_{G}\right)$, for all $w \in F P\left(G^{\wedge}\right)$, then we say that the groupoid $\mathbb{G}\left(\mathcal{A}_{G}\right)$ is a fractaloid. Equivalently, we say that the (labeled) graph groupoid $\mathbb{G}$ is a fractaloid.

Readers can understand the above definition of fractaloids as the graph-groupoid version of the fractal groups (See [1]). The following theorem provides the graphtheoretical characterization of fractaloids.

Theorem 4.2. Let $G$ be a canonical weighted graph with its labeled graph groupoid $\mathbb{G}$, and let $\mathcal{A}_{G}$ be the graph automaton induced by $G$ and $\mathcal{T}_{G}$, the $\mathcal{A}_{G}$-tree. Every w-part $\mathcal{T}_{w}$ of $\mathcal{T}_{G}$ is graph-isomorphic to $\mathcal{T}_{G}$, for all $w \in F P\left(G^{\wedge}\right)$, if and only if $\mathbb{G}$ is a fractaloid.

Proof. $(\Leftarrow)$ By definition, if the graph groupoid $\mathbb{G} \stackrel{\text { Groupoid }}{=} \mathbb{G}\left(\mathcal{A}_{G}\right)$ is a fractaloid, then, for any $w$-parts $\mathcal{T}_{w}$, the automata actions acting only on $\mathcal{T}_{w}$ generate the groupoids $\mathbb{G}\left(\mathcal{T}_{w}\right)$, which is groupoid-isomorphic to $\mathbb{G}$, where "Groupoid," means "being groupoid-isomorphic". Assume now that there exists a finite path $w_{0} \in F P\left(G^{\wedge}\right)$ such that the $w_{0}$-part $\mathcal{T}_{w_{0}}$ is not graph-isomorphic to the $\mathcal{A}_{G}$-tree $\mathcal{T}_{G}$. Then, clearly, the groupoids $\mathbb{G}\left(\mathcal{T}_{w_{0}}\right)$ and $\mathbb{G}\left(\mathcal{T}_{G}\right)$ are not groupoid-isomorphic. This contradicts our assumption.

$(\Rightarrow)$ Assume now that every $w$-part $\mathcal{T}_{w}$ of the $\mathcal{A}_{G}$-tree $\mathcal{T}_{G}$ is graph-isomorphic to $\mathcal{T}_{G}$, for all $w \in F P\left(G^{\wedge}\right)$. This shows that the groupoids $\mathbb{G}\left(\mathcal{T}_{w}\right)$ 's and $\mathbb{G}\left(\mathcal{T}_{G}\right)$ are groupoid-isomorphic, for all $w \in F P\left(G^{\wedge}\right)$, since the automata actions $\left\{\mathcal{A}_{y}\right\}$ acting on $\mathcal{T}_{w}$ and the automata action $\left\{\mathcal{A}_{d}\right\}$ acting on $\mathcal{T}_{G}$ are equivalent. Since $w$ is arbitrary in $F P\left(G^{\wedge}\right)$, the graph groupoid $\mathbb{G}$ is a fractaloid.

Let $G$ be a canonical weighted graph with its labeled graph groupoid $\mathbb{G}$, and assume that the automata actions $\left\{\mathcal{A}_{w}: w \in F P\left(G^{\wedge}\right)\right\}$ of the graph automaton $\mathcal{A}_{G}$ act fully on the $2 N$-regular tree $\mathcal{T}_{2 N}$, in the sense that the $\mathcal{A}_{G}$-tree $\mathcal{T}_{G}$, which is a full-subgraph of $\mathcal{T}_{2 N}$, is identical to $\mathcal{T}_{2 N}$. i.e., the automata actions of $\mathcal{A}_{G}$ act fully on $\mathcal{T}_{2 N}$, if $\mathcal{T}_{G} \stackrel{\text { Graph }}{=} \mathcal{T}_{2 N}$. 
Corollary 4.3. Let $G$ be a canonical weighted graph with its labeled graph groupoid $\mathbb{G}$, and let $\mathcal{A}_{G}$ be the graph automaton induced by $G$. If the automata actions $\left\{\mathcal{A}_{w}\right.$ $\left.: w \in F P\left(G^{\wedge}\right)\right\}$ of $\mathcal{A}_{G}$ act fully on the $2 N$-regular tree $\mathcal{T}_{2 N}$, then $\mathbb{G}$ is a fractaloid.

Proof. Suppose the automata actions act fully on the $2 N$-regular tree $\mathcal{T}_{2 N}$. Then, by definition, the $\mathcal{A}_{G}$-tree $\mathcal{T}_{G}$ is identical to $\mathcal{T}_{2 N}$. This shows that, every $w$-part $\mathcal{T}_{w}$ of $\mathcal{T}_{G}$ is graph-isomorphic to $\mathcal{T}_{2 N}$, for all $w \in F P\left(G^{\wedge}\right)$. Therefore, by the previous theorem, the graph groupoid $\mathbb{G}$ of $G$ is a fractaloid.

Also, the above corollary provide an easy technique to construct fractaloidal examples. For example, the one-flow circulant graphs and one-vertex-multi-loopedge graphs have their graph groupoids which are fractaloids, as connected "finite" directed graphs (See Section 4.2). Recall that a graph $G$ is finite, if $|V(G)|<\infty$ and $|E(G)|<\infty$.

How about the converse of the previous corollary? Let $\mathcal{T}$ be a one-flow growing infinite rooted tree with its root $v_{0}$. Then the terminal vertices of the incident edges of $v_{0}$ are called the 1-st level of $\mathcal{T}$. Similarly, if $v_{1}, \ldots, v_{t}$ are the vertices in the 1 -st level, then the terminal vertices of the incident edges of $v_{1}, \ldots, v_{t}$ are called the 2-nd level of $\mathcal{T}$. Inductively, we can decide the $n$-th level of $\mathcal{T}$, for $n \in \mathbb{N}$. For instance,

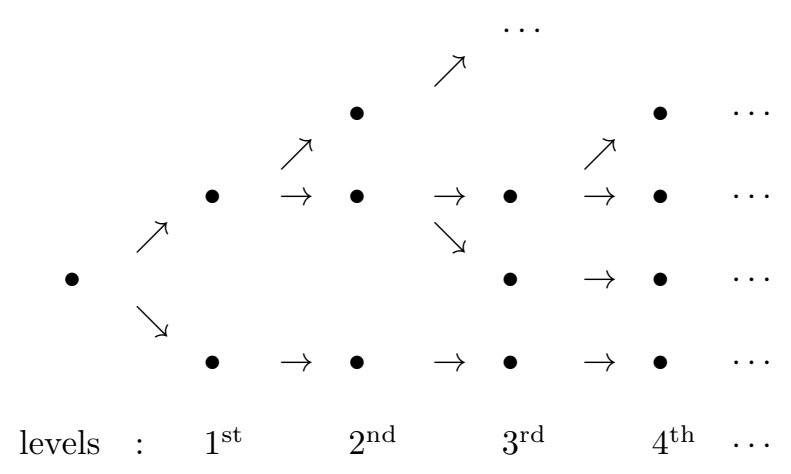

Theorem 4.4. If $\mathbb{G}$ is a fractaloid, then the automata actions $\left\{\mathcal{A}_{w}: w \in F P\left(G^{\wedge}\right)\right\}$ act fully on the $\mathcal{A}_{G}$-tree $\mathcal{T}_{G}$, and $\mathcal{T}_{G}$ is graph-isomorphic to the $2 N$-regular tree $\mathcal{T}_{2 N}$, by identifying their roots, where $N=\max \left\{\operatorname{deg}_{\text {out }}(v): v \in V(G)\right\}$.

Proof. By the graph-theoretical characterization of fractaloids, the labeled graph groupoid $\mathbb{G}$ is a fractaloid if and only if every $w$-part $\mathcal{T}_{w}$ of the $\mathcal{A}_{G}$-tree $\mathcal{T}_{G}$ is graphisomorphic to $\mathcal{T}_{G}$, for all $w \in F P\left(G^{\wedge}\right)$, by identifying their roots. Equivalently, for any vertices $v_{1}$ and $v_{2}$, the one-flow growing rooted trees $\mathcal{T}_{v_{1}}$ and $\mathcal{T}_{v_{2}}$ having their roots $\omega\left(v_{1}\right)$ and $\omega\left(v_{2}\right)$ are graph-isomorphic. Indeed, both $\mathcal{T}_{v_{1}}$ and $\mathcal{T}_{v_{2}}$ are graphisomorphic to $\mathcal{T}_{G}$, by identifying their roots, respectively, and hence the trees $\mathcal{T}_{v_{1}}$ and $\mathcal{T}_{v_{2}}$ are graph-isomorphic. 
Let $N=\max \left\{\operatorname{deg}_{\text {out }}(v): v \in V(G)\right\}$. Now, choose $v_{0} \in V(G)$ having its outdegree $\operatorname{deg}_{\text {out }}\left(v_{0}\right)=N$. Then we can take the $\mathcal{A}_{G}$-tree $\mathcal{T}_{G}$ as a one-flow growing rooted tree $\mathcal{T}_{v_{0}}$, having its root $\omega\left(v_{0}\right)=\left(\left(v_{0}, v_{0}\right), x_{0}\right)$. Remember that the tree $\mathcal{T}_{v_{0}}$ $=\mathcal{T}_{G}$ is a full-subgraph of the $2 N$-regular tree $\mathcal{T}_{2 N}$. By the connectedness of the shadowed graph $G^{\wedge}$ of the canonical weighted graph $G$, for any vertex $v \in V(G)$, we can find the finite path $w \in F P\left(G^{\wedge}\right)$ such that $w=v_{0} w v$ and $w^{-1}=v w v_{0}$. And since $\mathbb{G}$ is a fractaloid, the full-subgraph $\mathcal{T}_{v}$ of $\mathcal{T}_{v_{0}}=\mathcal{T}_{G}$ are graph-isomorphic. The existence of $w$ and $w^{-1}$, we can get that

$$
\omega(w)=\left(\left(v_{0}, v\right),\left(t_{i_{1}}, \ldots, t_{i_{n}}\right)\right)
$$

and

$$
\omega\left(w^{-1}\right)=\left(\left(v, v_{0}\right),\left(t_{i_{n}}, \ldots, t_{i_{1}}\right)\right)
$$

for some $n=|w| \in \mathbb{N}$. This shows that

$$
\operatorname{deg}_{\text {out }}^{\mathcal{T}_{G}}\left(\omega\left(v_{0}\right)\right)=\operatorname{deg}_{\text {out }}^{\mathcal{T}_{G}}\left(\left(v_{0}, v_{0}\right), x_{0}\right)=2 N
$$

where $\operatorname{deg}_{\text {out }}^{\mathcal{T}_{G}}($.$) means the out-degree of vertices of \mathcal{T}_{G}$. Equivalently, the root $\omega\left(v_{0}\right)$ of the fixed $\mathcal{A}_{G}$-tree $\mathcal{T}_{G}=\mathcal{T}_{v_{0}}$ has $(2 N)$-incident edges, which are the first level of the tree. This means that if $\operatorname{deg}_{\text {out }}\left(v_{0}\right)=N$, then the root $\omega\left(v_{0}\right)$ of $\mathcal{T}_{G}$ has $\operatorname{deg}_{\text {out }}^{\mathcal{T}_{G}}\left(v_{0}\right)=2 N$.

Let $v_{0}$ be given as before and let $\psi_{ \pm 1}, \ldots, \psi_{ \pm N}$ be the $2 N$-incident edges of the root $\omega\left(v_{0}\right)$ of $\mathcal{T}_{v_{0}}=\mathcal{T}_{G}$. For convenience, let $(\widetilde{p r} \circ \omega)\left(\psi_{k}\right)=t_{k}$, where $t_{k}=x_{k}$ if $k$ $\in\{1, \ldots, N\}$, and $t_{k}=-x_{k}$, if $k \in\{-1, \ldots,-N\}$. Then, for the terminal vertices

$$
\varphi_{k}=\varphi\left(\left(\left(v_{0}, v_{k}\right), t_{k}\right), \psi_{k}\right), \text { for } k= \pm 1, \ldots, \pm N
$$

create the full-subgraphs $\mathcal{T}_{v_{k}}$ 's of $\mathcal{T}_{v_{0}}$, having their roots $\varphi_{k}$, for all $k= \pm 1, \ldots$, $\pm N$, and they are graph-isomorphic to $\mathcal{T}_{v_{0}}$, since $\mathbb{G}$ is a fractaloid. Note that the full-subgraphs $\mathcal{T}_{v_{k}}$ 's are determined by the rooted one-flow growing tree induced by the 1-st level of $\mathcal{T}_{v_{0}}$. Thus, $\mathcal{T}_{v_{k}}$ 's have their 1-st level consisting of $2 N$-elements, for all $k= \pm 1, \ldots, \pm N$. Inductively, we can conclude that, for any $w \in F P\left(G^{\wedge}\right)$, the $w$-part $\mathcal{T}_{w}$ should have its 1 -st level consisting of $2 N$-elements. Equivalently, the tree $\mathcal{T}_{v_{0}}$ should be graph-isomorphic to the $2 N$-regular tree $\mathcal{T}_{2 N}$. Since $\mathcal{T}_{v_{0}}$ is our $\mathcal{A}_{G}$-tree $\mathcal{T}_{G}$, we can get that the $\mathcal{A}_{G}$-tree $\mathcal{T}_{G}$ is graph-isomorphic to the $2 N$-regular tree $\mathcal{T}_{2 N}$, by identifying their roots.

The above theorem shows that the converse of the previous corollary also holds true. Therefore, we can get the following other characterization of fractaloids. 
Corollary 4.5. Let $G$ be a canonical weighted graph with its labeled graph groupoid $\mathbb{G}$, and let $\mathcal{A}_{G}$ be the graph automaton induced by $G$. Then the $\mathcal{A}_{G}$-actions $\left\{\mathcal{A}_{w}\right.$ : $\left.w \in F P\left(G^{\wedge}\right)\right\}$ act fully on the $2 N$-regular tree $\mathcal{T}_{2 N}$, if and only if $\mathbb{G}$ is a fractaloid.

Proof. We know that the labeled graph groupoid $\mathbb{G}$ is a fractaloid if the $\mathcal{A}_{G}$-actions act fully on the $2 N$-regular tree $\mathcal{T}_{2 N}$, by the previous corollary. Now, by the previous theorem, we can conclude that, if $\mathbb{G}$ is a fractaloid, then the $\mathcal{A}_{G}$-tree $\mathcal{T}_{G}$ is identified with the $2 N$-regular tree $\mathcal{T}_{2 N}$. Therefore, if $\mathbb{G}$ is a fractaloid, then the $\mathcal{A}_{G}$-actions act fully on $\mathcal{T}_{2 N}$, since they act fully on $\mathcal{T}_{G}$.

\subsection{Examples.}

In this section, we consider some examples of fractaloids.

Example 4.1. Let $G$ be the one-flow circulant graph with three vertices. i.e., it is a graph with

$$
V(G)=\left\{v_{1}, v_{2}, v_{3}\right\}
$$

and

$$
E(G)=\left\{e_{j}=v_{j} e_{j} v_{j+1}: j=1,2,3, v_{4} \stackrel{\text { def }}{=} v_{1}\right\}
$$

Then we have $\max \left\{\operatorname{deg}_{\text {out }}\left(v_{j}\right): j=1,2,3\right\}=1$. So, as in Section 3.1, we can decide the labeling set $\{0,1\}$, and the labeled graph groupoid $\mathbb{G}$ of $G$, labeled by $\{0$, $\pm 1\}^{*}$. Then the graph automaton $\mathcal{A}_{G}$ of $G$ is determined and the automata actions of $\mathcal{A}_{G}$ act fully on the 2-regular tree $\mathcal{T}_{2}$, by the existence of the pairs $\left(\mathcal{A}_{e_{1}}, \mathcal{A}_{e_{3}^{-1}}\right)$, $\left(\mathcal{A}_{e_{2}}, \mathcal{A}_{e_{1}^{-1}}\right)$ and $\left(\mathcal{A}_{e_{3}}, \mathcal{A}_{e_{2}^{-1}}\right)$ of actions. Therefore, the graph groupoid $\mathbb{G}$ of $G$ is a fractaloid.

By the inductive modification of the previous example, we can get the following proposition.

Proposition 4.6. Let $G$ be a one-flow circulant graph with $N$-vertices with $V(G)$ $=\left\{v_{1}, \ldots, v_{N}\right\}$ and $E(G)=\left\{e_{j}=v_{j} e_{j} v_{j+1}: j=1, \ldots, N, v_{N+1} \stackrel{\text { def }}{=} v_{1}\right\}$. Then the graph groupoid $\mathbb{G}$ of $G$ is a fractaloid.

The following example is about certain fractal groups.

Example 4.2. Let $G$ be the one-vertex- $N$-loop-edge graph with $V(G)=\{v\}$ and $E(G)=\left\{e_{j}=v e_{j} v: j=1, \ldots, N\right\}$. In [10] and [11], we showed that the graph groupoid $\mathbb{G}$ of $G$ is a group which is group-isomorphic to the free group $F_{N}$ with $N$-generators. The free group $F_{N}$ is a fractal group, for $N \in \mathbb{N}$ (See [1]). And hence, the graph groupoid $\mathbb{G}$ is a fractaloid which is a fractal group. 
By the previous example, we can get the following proposition.

Proposition 4.7. Let $G$ be a one-vertex-multi-loop-edge graph. Then the graph groupoid $\mathbb{G}$ of $G$ is a fractaloid.

Recall that, we say a directed graph $G$ is finite, if $|V(G)|<\infty$, and $|E(G)|<$ $\infty$. The previous two examples show that it is possible that, even though the given graph is finite, its graph groupoid can be a fractaloid.

The following example is trivial. It shows that the automata actions induced by the $N$-regular tree $\mathcal{T}_{N}$ act fully on the $2 N$-regular tree $\mathcal{T}_{2 N}$, and hence they generate a fractaloid.

Example 4.3. Let $\mathcal{T}_{N}$ be the $N$-regular tree. Then it induces the graph automaton $\mathcal{A}_{T_{N}}$, and the automata actions act fully on the $2 N$-regular tree $\mathcal{T}_{2 N}$. Therefore, the graph groupoid $\mathbb{T}_{N}$ of $\mathcal{T}_{N}$ is a fractaloid.

Example 4.4. Every infinitely countable linear graph $L$ induces a fractaloid $\mathbb{L}$. i.e., the graph $L$ is graph-isomorphic to the following graph,

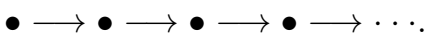

Then the graph $L$ induces the graph automaton $\mathcal{A}_{L}$. Since the (non-reduced) finite path set $F P\left(L^{\wedge}\right)$ of the shadowed graph $L^{\wedge}$ is bijective to the finite path set $F P\left(\mathcal{T}_{2}\right)$ of 2-regular tree $\mathcal{T}_{2}$. This guarantees that automata actions of $\mathcal{A}_{L}$ act fully on $\mathcal{T}_{2}$. Therefore, $\mathbb{L}$ is a fractaloid. Indeed, the graph $L$ is regarded as the 1-regular tree, and hence, by the previous example, it induces a fractaloid.

\section{LABEling Operators of Fractaloids}

Let $G$ be a canonical weighted graph with its labeled graph groupoid $\mathbb{G}$, and let

$$
N=\max \left\{\operatorname{deg}_{\text {out }}(v): v \in V(G)\right\} \text {. }
$$

We will use the same notations used in the previous Sections. In Section 2.4, we defined right graph von Neumann algebras $\mathbb{M}_{G}=M \times_{\beta} \mathbb{G}$, where $M$ is an arbitrary fixed von Neumann algebra, contained in the operator algebra $B(K)$, and $\beta$ is a right $G$-representation, which is an intertwined groupoid action of $\mathbb{G}$, acting on the Hilbert space $K \otimes H_{G}$. As we mentioned in Section 2.4, we are only interested in 
the case where $M=\mathbb{C}$. Then the right graph von Neumann algebras $\mathbb{M}_{G}=\mathbb{C} \times \beta$ $\mathbb{G}$, for all $\beta$, are $*$-isomorphic to the groupoid von Neumann algebra

$$
\overline{\mathbb{C}[\mathbb{G}}^{w}=\overline{\mathbb{C}[\beta(\mathbb{G})}^{w}, \text { for all } \beta,
$$

as a $W^{*}$-subalgebra of $B\left(H_{G}\right)$, where $H_{G}$ is the graph Hilbert space induced by the graph $G$.

Notation From now, if we denote $\mathbb{M}_{G}$, then it is automatically assumed to be the $W^{*}$-subalgebra $\overline{\mathbb{C}[\mathbb{G}]}$ of $B\left(H_{G}\right)$. And we call it "the" right graph von Neumann algebra induced by $G$.

\subsection{Labeling Operators.}

Now, we will define certain operators on the graph Hilbert space $H_{G}$. Then we can easily realize that these operators are elements of $\mathbb{M}_{G}$.

Definition 5.1. Let $G$ be a canonical weighted graph and let $H_{G}$ be the corresponding graph Hilbert space. Define an operator $T_{i}$ on $H_{G}$ by

$$
T_{i}\left(\xi_{w}\right) \stackrel{\text { def }}{=} \xi_{w} \xi_{e}=\xi_{w e}=\left\{\begin{array}{cl} 
& \text { if } \exists e \in E\left(G^{\wedge}\right) \text { s.t. } \\
& w e \neq \emptyset \text { and } \\
\xi_{w e} & \omega(e)=\left(\left(v, v^{\prime}\right), t_{i}\right) \\
0 & \text { otherwise, }
\end{array}\right.
$$

for all $\xi_{w} \in \mathcal{B}_{H_{G}}$, with $w=w v$ in $F P_{r}\left(G^{\wedge}\right)$, and for all $i= \pm 1, \ldots, \pm N$, where $\mathcal{B}_{H_{G}}$ is the Hilbert basis of $H_{G}$. Here, $t_{i}=x_{i}$ if $t_{i} \in X$, and $t_{i}=-x_{i}$, if $t_{i} \in-X$. The operators $T_{k}$ 's are called the $k$-th labeling operators, for $k= \pm 1, \ldots, \pm N$. The operator $T_{G}$ on $H_{G}$ is said to be the labeling operator, if

$$
T_{G} \stackrel{\text { def }}{=} \sum_{j=-N}^{-1} T_{j}+\sum_{i=1}^{N} T_{i}
$$

The labeling operator is similar to the Hecke-type operators (e.g., [35]) or the Ruelle operators (e.g., [31] and [32]) or the radial operators (e.g., [36]) The definition shows that each labeling $t_{k} \in \pm X$ generates the $k$-th labeling operator $T_{k}$ on $H_{G}$, for $k= \pm 1, \ldots, \pm N$. And the labeling set $\pm X=\left\{ \pm x_{1}, \ldots, \pm x_{N}\right\}$, itself, generates the labeling operator $T_{G}$ on $H_{G}$.

By the very definition, we can realize that the $k$-th labeling operators $T_{k}$ 's, for $k$ $= \pm 1, \ldots, \pm N$, and the labeling operator $T_{G}$ of $\mathbb{G}$ are contained in the right graph von Neumann algebra $\mathbb{M}_{G}$. 
Lemma 5.1. Let $G$ be a canonical weighted graph with its labeled graph groupoid $\mathbb{G}$, and let $\mathbb{M}_{G}$ be the right graph von Neumann algebra induced by $G$. Also, let $T_{k}$ 's and $T_{G}$ be the $k$-th labeling operators and the labeling operator of $\mathbb{G}$ on the graph Hilbert space $H_{G}$, where $k= \pm 1, \ldots, \pm N$. Then they are contained in $\mathbb{M}_{G}$.

Proof. Let $\mathbb{M}_{G}$ be the right graph von Neumann algebra induced by $G$. Construct the elements $\tau_{k}$ and $\tau$ of $\mathbb{M}_{G}$ :

$$
\tau_{k}=\sum_{e \in E\left(G^{\wedge}\right),(\overline{p r} \circ \omega)(e)=t_{k}}^{(N)} R_{e}
$$

where

$$
t_{k} \stackrel{\text { def }}{=} \begin{cases}x_{k} & \text { if } k=1, \ldots, N \\ -x_{k} & \text { if } k=-1, \ldots,-N,\end{cases}
$$

for all $k= \pm 1, \ldots, \pm N$. Then these elements $\tau_{k}$ 's are identified with the $k$-th labeling operators $T_{k}$, on the graph Hilbert space $H_{G}$, for all $k= \pm 1, \ldots, \pm N$. Therefore, the $k$-th labeling operators $T_{k}$ 's are contained in $\mathbb{M}_{G}$, for all $k$.

Define now the element $\tau$ of $\mathbb{M}_{G}$ by

$$
\tau=\sum_{i=-N}^{-1} \tau_{i}+\sum_{j=1}^{N} \tau_{j}
$$

where $\tau_{k}$ 's are defined as above, for all $k= \pm 1, \ldots, \pm N$. Then, by definition, $\tau$ is identified with $T_{G}$ on $H_{G}$.

By the previous lemma, we can regard the labeling operators $T_{k}$ 's and $T_{G}$ as elements of the right graph von Neumann algebra $\mathbb{M}_{G}$.

Corollary 5.2. Let $T_{G}$ be the labeling operator of $\mathbb{G}$ on $H_{G}$. Then it is identified with the operator $\sum_{e \in E\left(G^{\wedge}\right)} R_{e}$ generated by all edges of the shadowed graph $\hat{G}^{\wedge}$.

By the previous lemma, we can get the following adjoint property of the $k$-th labeling operators $T_{k}$ 's.

Lemma 5.3. Let $k \in\{ \pm 1, \ldots, \pm N\}$ be fixed, and let $T_{k}$ be the $k$-th labeling operator on $H_{G}$. Then the adjoint $T_{k}^{*}$ of $T_{k}$ is identical to the $(-k)$-th labeling operator $T_{-k}$.

Proof. By the previous lemma, we can consider the $k$-th labeling operator $T_{k}$ as an element in $\mathbb{M}_{G}$. i.e., let 


$$
T_{k}=\sum_{e \in E\left(G^{\wedge}\right),(\widetilde{p r} \circ \omega)(e)=t_{k}} R_{e} \text { in } \mathbb{M}_{G} .
$$

Then we can have that

$$
\begin{aligned}
& T_{k}^{*}=\sum_{e \in E\left(G^{\wedge}\right),(\widetilde{p r} \circ \omega)(e)=t_{k}} R_{e}^{*}=\sum_{e \in E\left(G^{\wedge}\right),(\widetilde{p r} \circ \omega)(e)=t_{k}} R_{e^{-1}} \\
& =\sum_{e^{-1} \in E\left(G^{\wedge}\right),(\widetilde{p r} \circ \omega)\left(e^{-1}\right)=-t_{k}} R_{e^{-1}}=\sum_{e \in E\left(G^{\wedge}\right),(\widetilde{p r} \circ \omega)(e)=-t_{k}} R_{e}=T_{-k} .
\end{aligned}
$$

Indeed, by the canonical weighting process, for any $e \in E\left(G^{\wedge}\right), \widetilde{p r}(\omega(e))=t_{k}$ if and only if $\widetilde{p r}\left(\omega\left(e^{-1}\right)\right)=-t_{k}$, where $t_{k} \in \pm X$.

Recall that, by Section 2.5, [10] and [11], we know that two elements $w_{1}$ and $w_{2}$ of a graph groupoid $\mathbb{G}$ are diagram-distinct, in the sense that (i) $w_{1} \neq w_{2}^{-1}$, and (ii) the diagrams (which are the graphical images) of $w_{1}$ and $w_{2}$ (in $\mathbb{R}^{2}$ ) are distinct, if and only if the right multiplication operators $R_{w_{1}}$ and $R_{w_{2}}$ are free over $\mathbb{D}_{G}$ in $\left(\mathbb{M}_{G}, E\right)$, where $\mathbb{D}_{G}$ is the $\mathbb{C}$-diagonal subalgebra of $\mathbb{M}_{G}$ and $E$ is the canonical conditional expectation of $\mathbb{M}_{G}$ onto $\mathbb{D}_{G}$. Thus, more precisely, two subsets $X_{1}$ and $X_{2}$ of $\mathbb{G}$ are diagram-distinct, in the sense that, for any pair $\left(w_{1}, w_{2}\right) \in X_{1} \times X_{2}$, the elements $w_{1}$ and $w_{2}$ are diagram-distinct if and only if the operators $\sum_{w_{1} \in X_{1}}$ $R_{w_{1}}$ and $\sum_{w_{2} \in X_{2}} R_{w_{2}}$ are free over $\mathbb{D}_{G}$ in $\left(\mathbb{M}_{G}, E\right)$. So, we can get the following proposition.

Proposition 5.4. The operators $T_{k}+T_{-k} \in \mathbb{M}_{G}$, for $k=1, \ldots, N$, are free over $\mathbb{D}_{G}$ from each other in $\left(\mathbb{M}_{G}, E\right)$, where $T_{k}$ 's are the $k$-th labeling operators, for $k$ $= \pm 1, \ldots, \pm N$.

Proof. Let's denote the operators $T_{k}+T_{-k}$ by $S_{k}$, for $k=1, \ldots, N$. And, for the fixed $k$, construct the set $\mathcal{S}_{k}$ by

$$
\mathcal{S}_{k}=\left\{e \in E\left(G^{\wedge}\right):(\widetilde{p r} \circ \omega)(e)=x_{k} \text { or }-x_{k}\right\},
$$

for $k=1, \ldots, N$. Then the operator $S_{k}$ can be re-written by

$$
S_{k}=\sum_{e \in \mathcal{S}_{k}} R_{e}, \text { for all } k=1, \ldots, N
$$

By the canonical weighting process, the sets $\mathcal{S}_{k}$ 's are diagram-distinct from each other, for all $k=1, \ldots, N$. Therefore, the operators $S_{k}$ 's are free over $\mathbb{D}_{G}$ from each other in $\left(\mathbb{M}_{G}, E\right)$. 
Corollary 5.5. (1) The labeling operator $T_{G}$ of $\mathbb{G}$ on $H_{G}$ is a $\mathbb{D}_{G}$-free sum of the $\mathbb{D}_{G}$-free elements $T_{k}+T_{-k}$, in $\mathbb{M}_{G}$, for all $k=1, \ldots, N$.

(2) The labeling operator $T_{G}$ is self-adjoint on $H_{G}$.

\subsection{Amalgamated Free Distributional Data of Labeling Operators.}

Let $B \subset A$ be von Neumann algebras with $1_{B}=1_{A}$, and assume that there exists a conditional expectation $E_{B}: A \rightarrow B$. Then the pair $\left(A, E_{B}\right)$ is a $B$-valued $W^{*}$-probability space. Let $a \in\left(A, E_{B}\right)$ be a $B$-valued random variable. Then the $B$-valued free distributional data of $a$ is characterized by the $B$-valued joint *-moments

$$
\cup_{n=1}^{\infty}\left\{\begin{array}{l|l}
E_{B}\left(b_{1} a^{r_{1}} b_{2} a^{r_{2}} \ldots b_{n} a^{r_{n}}\right) \mid \begin{array}{c}
\left(r_{1}, \ldots, r_{n}\right) \in\{1, *\}^{n} \\
b_{1}, \ldots, b_{n} \in B \text { arbitrary }
\end{array}
\end{array}\right\} .
$$

i.e., the above $B$-values generate the $B$-valued free distribution $\rho_{a}$ of $a$ (See [5], [12] and [21]). Suppose now that the operator $a$ is self-adjoint. Then its $B$-valued free distribution $\rho_{a}$ is completely determined by the $B$-valued moments of $a$,

$$
\left\{\begin{array}{c|c}
E_{B}\left(b_{1} a b_{2} a \ldots b_{n} a\right): \mid \begin{array}{c}
n \in \mathbb{N}, \text { and } \\
b_{1}, \ldots, b_{n} \in B \text { arbitrary }
\end{array}
\end{array}\right\}
$$

Since the labeling operator $T_{G}$ of $\mathbb{G}$ on the graph Hilbert space $H_{G}$ is an element in the right graph von Neumann algebra $\mathbb{M}_{G}$, we can consider its $\mathbb{D}_{G}$-valued free distributional data, by observing the $\mathbb{D}_{G}$-valued moments $\left\{E\left(T_{G}^{n}\right): n \in \mathbb{N}\right\}$. Since

$$
T_{G}=\left(\sum_{i=-N}^{-1} T_{i}\right)+\left(\sum_{j=1}^{N} T_{j}\right),
$$

where $T_{k}$ 's are the $k$-th labeling operators, for $k= \pm 1, \ldots, \pm N$, we can have that

$$
\begin{aligned}
& E\left(T_{G}^{n}\right)=\sum_{\left(i_{1}, \ldots, i_{n}\right) \in\{ \pm 1, \ldots, \pm N\}^{n}} E\left(T_{i_{1}} \ldots T_{i_{n}}\right) \\
& =\sum_{\left(i_{1}, \ldots, i_{n}\right) \in\{ \pm 1, \ldots, \pm N\}^{n}}\left(\sum_{\left(e_{1}, \ldots, e_{n}\right) \in E\left(G^{n}\right)^{n},(\widetilde{p r} \circ \omega)\left(e_{j}\right)=t_{i_{j}}}\left(E\left(R_{e_{1}} \ldots R_{e_{n}}\right)\right)\right),
\end{aligned}
$$

for all $n \in \mathbb{N}$. 


\subsection{Labeling Operators of Fractaloids.}

In this section, we will consider the $\mathbb{D}_{G}$-valued moments of the labeling operator $T_{G}$ of $\mathbb{G}$ in $\mathbb{M}_{G}$, where $\mathbb{G}$ is a fractaloid. We showed that, in general, if $T_{G}$ is the labeling operator of an arbitrary labeled graph groupoid $\mathbb{G}$ in the right graph von Neumann algebra $\mathbb{M}_{G}$, then the $n$-th $\mathbb{D}_{G}$-valued moment $E\left(T_{G}^{n}\right)$ is determined by (5.1).

Now, we are interested in the more precise formula than (5.1), when we have a fractaloid $\mathbb{G}$. If we can find the more precise formula, then we not only explain the $\mathbb{D}_{G}$-valued free distributional data of $T_{G}$, but also show how the fractal property works on graph groupoids (and hence on graph Hilbert spaces). Moreover, it also shows how the admissibility of fractaloids works inside the right graph von Neumann algebra $\mathbb{M}_{G}$. Therefore, the study of the $\mathbb{D}_{G}$-valued free distributional data of the labeling operator $T_{G}$ of a fractaloid $\mathbb{G}$ is for studying the groupoidal-and-operatoralgebraic fractal properties.

From now, all graph groupoids in this Section are fractaloids.

Notation For convenience, we will denote the map $\widetilde{p r} \circ \omega$ simply by $\widetilde{\omega}$. i.e.,

defined by

$$
\widetilde{\omega}: \mathbb{F}^{+}\left(G^{\wedge}\right) \rightarrow\left( \pm X_{0}^{*}\right)
$$

$$
\widetilde{\omega}(w)= \begin{cases}x_{0} & \text { if } w \in V(G) \\ \widetilde{p r}(\omega(w)) & \text { if } w \in E\left(G^{\wedge}\right) \\ \left(\widetilde{\omega}\left(e_{1}\right), \ldots, \widetilde{\omega}\left(e_{k}\right)\right) & \text { if } w=e_{1} \ldots e_{k} \in F P\left(G^{\wedge}\right), \\ & \text { for } k \geq 2 \\ \emptyset_{*} & \text { if } w=\emptyset,\end{cases}
$$

for all $w \in \mathbb{F}^{+}\left(G^{\wedge}\right)$. Recall the operations $\theta$ and $\omega_{+}$defined in Section 3.2.

By Section 4.1, we have the following characterization of fractaloids: the labeled graph groupoid $\mathbb{G}$ is a fractaloid if and only if the automata actions $\left\{\mathcal{A}_{w}: w \in\right.$ $\left.F P\left(G^{\circ}\right)\right\}$ of the graph automaton $\mathcal{A}_{G}$ act fully on the $2 N$-regular tree $\mathcal{T}_{2 N}$. So, if $T_{G}$ is the labeling operator of a fractaloid $\mathbb{G}$ in the right graph von Neumann algebra $\mathbb{M}_{G}$, then the formula (5.1) is re-formulated as follows:

$$
E\left(T_{G}^{n}\right)=\sum_{\left(i_{1}, \ldots, i_{n}\right) \in\{ \pm 1, \ldots, \pm N\}^{n}}\left(\sum_{e_{1} \ldots e_{n} \in F P\left(G^{\wedge}\right), \widetilde{\omega}\left(e_{j}\right)=t_{i_{j}}} E\left(R_{e_{1} \ldots e_{n}}\right)\right)
$$


by $(5.1)$

$$
\begin{aligned}
& =\sum_{\left(i_{1}, \ldots, i_{n}\right) \in\{ \pm 1, \ldots, \pm N\}^{n}}\left(\sum_{e_{1} \ldots e_{n} \in F P\left(G^{\wedge}\right), e_{1} \ldots e_{n} \in V(G) \subset \mathbb{G}, \widetilde{\omega}\left(e_{j}\right)=t_{i_{j}}} E\left(R_{e_{1} \ldots e_{n}}\right)\right) \\
& =\sum_{\left(i_{1}, \ldots, i_{n}\right) \in\{ \pm 1, \ldots, \pm N\}^{n}}\left(\begin{array}{l}
\sum_{v \in V(G) \subset \mathbb{G}, v=e_{1} \ldots e_{n} \in F P\left(G^{\wedge}\right), \widetilde{\omega}\left(e_{j}\right)=t_{i_{j}}} \\
\sum_{v}
\end{array}\right)
\end{aligned}
$$

since $E\left(R_{v}\right)=R_{v}$, for all $v \in V(G) \subset \mathbb{G}$

$$
=\sum_{\left(i_{1}, \ldots, i_{n}\right) \in\{ \pm 1, \ldots, \pm N\}^{n}}\left(\sum_{e_{1} \ldots e_{n} \in F P\left(G^{\wedge}\right), \widetilde{\omega}\left(e_{j}\right)=t_{i_{j}}, \sum_{k=1}^{n} t_{i_{k}}=x_{0}} R_{e_{1} \ldots e_{n}}\right),
$$

by Section 3.2: Remember that a finite path $e_{1} \ldots e_{n}=\begin{array}{llllll}v & e_{1} & \ldots & e_{n} & v & \text { in }\end{array}$ $F P\left(G^{\wedge}\right)$, with $v \in V\left(G^{\wedge}\right)$, is the vertex $v$ in the labeled graph groupoid $\mathbb{G}$ (under the reduction), where $e_{j} \in E\left(G^{\wedge}\right)$ with $\widetilde{\omega}\left(e_{j}\right)=t_{i_{j}}$, if and only if $\sum_{j=1}^{n} t_{i_{j}}=x_{0}$ in $\mathbb{C}^{\oplus N}$, in general.

Fix now a vertex $v \in V(G)$. Define a subset $\mathcal{F}_{v, n}$ of the finite path set $F P\left(G^{\wedge}\right)$ of the shadowed graph $G^{\wedge}$ by

$$
\mathcal{F}_{v, n} \stackrel{\text { def }}{=}\left\{\begin{array}{lll}
e_{1} \ldots e_{n} \in F P\left(G^{\wedge}\right) & \begin{array}{c}
e_{1}=v e_{1} \text { and } \\
\sum_{j=1}^{n} \widetilde{\omega}\left(e_{j}\right)=x_{0}
\end{array}
\end{array}\right\}
$$

for all $n \in \mathbb{N}$. i.e., the sets $\mathcal{F}_{v, n}$ are the collection of all length- $n$ finite paths of $F P\left(G^{\wedge}\right)$ which are all identified with the vertex $v$ in the graph groupoid $\mathbb{G}$, under the reduction $(\mathrm{RR})$, for all $n \in \mathbb{N}$. We will say that the set $\mathcal{F}_{v, n}$ is the length-n$v$-subset of $F P\left(G^{\wedge}\right)$, for all $n \in \mathbb{N}$. Remark that the weighting (or the labeling) process makes us understand the admissibility and the reduction on $\mathcal{F}_{v, n}$ 's (inside $\mathbb{G})$, more in detail, for all $v \in V(G)$ and $n \in \mathbb{N}$. Again, by the labeling process, we can re-express the sets $\mathcal{F}_{v, n}$ 's as follows,

$$
\mathcal{F}_{v, n}=\cup_{\left(i_{1}, \ldots, i_{n}\right) \in\{ \pm 1, \ldots, \pm N\}^{n}}\left\{\begin{array}{l|l}
e_{1} \ldots e_{n} \in F P\left(G^{\wedge}\right) & \begin{array}{c}
e_{1}=v e_{1} \in E\left(G^{\wedge}\right) \\
\widetilde{\omega}\left(e_{j}\right)=t_{i_{j}}, \text { for } j \\
\sum_{j=1}^{n} t_{i_{j}}=x_{0}
\end{array}
\end{array}\right\}
$$

for all $n \in \mathbb{N}$. Let $v_{1}$ and $v_{2}$ be arbitrarily chosen vertices in $V(G)$. Then we can have the corresponding sets $\left\{\mathcal{F}_{v_{1}, n}\right\}_{n=1}^{\infty}$ and $\left\{\mathcal{F}_{v_{2}, n}\right\}_{n=1}^{\infty}$. Then, for each $n \in \mathbb{N}$, the cardinalities $\left|\mathcal{F}_{v_{1}, n}\right|$ and $\left|\mathcal{F}_{v_{2}, n}\right|$ are identical, whenever $\mathbb{G}$ is a fractaloid. 
Lemma 5.6. Let $\mathbb{G}$ be a given fractaloid, and let $\mathcal{F}_{v, n}$ be the length-n-v-subsets of $F P\left(G^{\wedge}\right)$, for all $v \in V(G)$ and $n \in \mathbb{N}$. Then, for any $n \in \mathbb{N}$, the cardinalities $\left|\mathcal{F}_{v, n}\right|$ are identical, for all $v \in V(G)$.

Proof. Let $v_{1} \neq v_{2}$ be the distinct vertices in $V(G)$, and let $\mathcal{F}_{v_{1}, n}$ and $\mathcal{F}_{v_{2}, n}$ be the length- $n$-vertex-subsets of $v_{1}$ and $v_{2}$ in $F P\left(G^{\wedge}\right)$, respectively, for $n \in \mathbb{N}$. By definition,

$$
\mathcal{F}_{v_{k}, n}=\bigcup_{\left(i_{1}, \ldots, i_{n}\right) \in\{ \pm 1, \ldots, \pm N\}^{n}}\left\{\begin{array}{l|l}
e_{1} \ldots e_{n} \in F P\left(G^{\wedge}\right) & \begin{array}{c}
e_{1}=v_{k} e_{1} \in E\left(G^{\wedge}\right) \\
\widetilde{\omega}\left(e_{j}\right)=t_{i_{j}}, \text { for } j, \\
\sum_{j=1}^{n} t_{i_{j}}=x_{0}
\end{array}
\end{array}\right\},
$$

for $k=1,2$. Define now the map

$$
\mathcal{E}: E\left(G^{\wedge}\right) \rightarrow\left\{\mathcal{A}_{e}: e \in E\left(G^{\wedge}\right)\right\}
$$

by

$$
\mathcal{E}(e)=\mathcal{A}_{e}, \text { for all } e \in E\left(G^{\wedge}\right) .
$$

Clearly, this map $\mathcal{E}$ is bijective and hence it is extendable to the bijective map, also denoted by $\mathcal{E}$, from $F P\left(G^{\wedge}\right)$ onto $\left\{\mathcal{A}_{w}: w \in F P\left(G^{\wedge}\right)\right\}$. The existence of this bijection $\mathcal{E}$ shows that we can regard the elements in $\mathcal{F}_{v_{k}, n}$ as the $\mathcal{E}$-corresponding elements on $F P\left(\mathcal{T}_{2 N}\right)$, where $\mathcal{T}_{2 N}$ is the $2 N$-regular tree where the automata actions $\mathcal{A}_{w}$ 's act. (Remember that the $\mathcal{A}_{G}$-tree $\mathcal{T}_{G}$ is identical to $\mathcal{T}_{2 N}$, whenever $\mathbb{G}$ is a fractaloid!) Since the graph groupoid $\mathbb{G}$ is a fractaloid, $\mathcal{E}\left(\mathcal{F}_{v_{k}, n}\right)$ create the fullsubgraphs $G_{v_{k}, n}$, having its finite path set $F P\left(G_{v_{k}, n}\right)=\mathcal{E}\left(\mathcal{F}_{v_{k}, n}\right)$ in $\mathcal{T}_{2 N}$, and they are graph-isomorphic, for $k=1,2$, and for $n \in \mathbb{N}$. Therefore, the generating sets $\mathcal{F}_{v_{k}, n}$, which are regarded as the edge sets of the full-subgraphs $G_{v_{k}, n}$, should have the same cardinality.. i.e.,

$$
\left|\mathcal{E}\left(\mathcal{F}_{v_{1}, n}\right)\right|=\left|\mathcal{E}\left(\mathcal{F}_{v_{2}, n}\right)\right| \Longleftrightarrow\left|\mathcal{F}_{v_{1}, n}\right|=\left|\mathcal{F}_{v_{2}, n}\right|,
$$

by the bijectivity of $\mathcal{E}$, for all $n \in \mathbb{N}$. Since $v_{1}$ and $v_{2}$ are arbitrary, for any $n \in$ $\mathbb{N}$, the cardinalities $\left|\mathcal{F}_{v, n}\right|$ of the length- $n$ - $v$-subsets of $F P\left(G^{\wedge}\right)$ are identical, for all $v \in V(G)$.

By the previous lemma, we can re-formulate the formula (5.1), where the given labeled graph groupoid $\mathbb{G}$ is a fractaloid.

Theorem 5.7. Let $G$ be a canonical weighted graph with its labeled graph groupoid $\mathbb{G}$, and assume that $\mathbb{G}$ is a fractaloid. Then the labeling operator $T_{G}$ of $\mathbb{G}$ in $\mathbb{M}_{G}$ satisfies that

$$
E\left(T_{G}^{n}\right)=\left|\sum_{n}^{(N)}\right| \cdot 1_{\mathbb{D}_{G}},
$$


where

(5.4)

$$
\sum_{n}^{(N)}=\left\{\left(t_{i_{1}}, \ldots, t_{i_{n}}\right) \in( \pm X)^{n} \mid \sum_{j=1}^{n} t_{i_{j}}=x_{0}\right\},
$$

for all $n \in \mathbb{N}$.

Proof. By (5.2) and by the previous lemma, we can compute, for any fixed $n \in \mathbb{N}$,

$$
\begin{aligned}
E\left(T_{G}^{n}\right) & =\sum_{\left(i_{1}, \ldots, i_{n}\right) \in\{ \pm 1, \ldots, \pm N\}^{n}}\left(\sum_{e_{1} \ldots e_{n} \in F P\left(G^{\wedge}\right), \widetilde{\omega}\left(e_{j}\right)=t_{i_{j}}, \sum_{k=1}^{n} t_{i_{k}}=x_{0}} R_{e_{1} \ldots e_{n}}\right) \\
& =\sum_{v \in V(G)}\left|\mathcal{F}_{v, n}\right| R_{v}=\sum_{v \in V(G)} \eta_{n} R_{v}=\eta_{n}\left(\sum_{v \in V(G)} R_{v}\right) .
\end{aligned}
$$

The last two equalities hold, by the previous lemma. i.e., for the fixed $n \in \mathbb{N}$, the cardinalities $\left|\mathcal{F}_{v, n}\right|$ of the length- $n$ - $v$-subsets $\mathcal{F}_{v, n}$ are identical to the number $\eta_{n}$, for all $v \in V(G)$, whenever $\mathbb{G}$ is a fractaloid. Recall that $\sum_{v \in V(G)} R_{v}$ is the identity element $1_{\mathbb{D}_{G}}=1_{\mathbb{M}_{G}}$ of $\mathbb{D}_{G} \subseteq \mathbb{M}_{G}$. Therefore, we have that

$$
E\left(T_{G}^{n}\right)=\eta_{n} \cdot 1_{\mathbb{D}_{G}} \in \mathbb{D}_{G}, \text { for all } n \in \mathbb{N},
$$

for some scalar-values $\left\{\eta_{n}\right\}_{n=1}^{\infty}$. Notice that

$$
\eta_{n}=\left|\mathcal{F}_{v, n}\right|, \text { for all } v \in V(G)
$$

for any $n \in \mathbb{N}$. By the definition of length- $n$-vertex-subsets $\mathcal{F}_{v}$, $n$ 's of $F P\left(G^{\wedge}\right)$, we can construct sets $\sum_{n}^{(N)}$ (independent from the choice of vertices) by

$$
\sum_{n}^{(N)} \stackrel{\text { def }}{=}\left\{\left(t_{i_{1}}, \ldots, t_{i_{n}}\right) \in( \pm X)^{n} \mid \sum_{j=1}^{n} t_{i_{j}}=x_{0}\right\}
$$

for all $n \in \mathbb{N}$. Then the cardinality $\left|\sum_{n}^{(N)}\right|$ of $\sum_{n}^{(N)}$ is identical to the value $\eta_{n}$, for all $n \in \mathbb{N}$.

Remark 5.1. In general, without the assumption that $\mathbb{G}$ is a fractaloid, the equalities

$$
\eta_{n}=\left|\mathcal{F}_{v, n}\right| \quad \text { and } \quad \eta_{n}=\left|\sum_{n}^{(N)}\right| \text {, }
$$


in the proof of the previous theorem, do not hold. (See [40].)

The above theorem shows that the $\mathbb{D}_{G}$-valued (or the operator-valued) moments $E\left(T_{G}^{n}\right)$ of the labeling operator $T_{G}$ are completely determined by the scalar-values $\left|\sum_{n}^{(N)}\right|$ which are the cardinalities of certain sets $\sum_{n}^{(N)}$, for all $n \in \mathbb{N}$, whenever the labeled graph groupoid $\mathbb{G}$ is a fractaloid. Also, the above theorem shows how the labeling (in terms of the admissibility of $\mathbb{G}$ ) works (in $\mathbb{G}$ ) and $\mathbb{M}_{G}$, whenever $\mathbb{G}$ is a fractaloid.

\subsection{Refinements of (5.3).}

In Section 5.3, we showed that the $\mathbb{D}_{G}$-valued free moments $\left\{E\left(T_{G}^{n}\right)\right\}_{n=1}^{\infty}$ of the labeling operator $T_{G}$ of a fractaloid $\mathbb{G}$ in the right graph von Neumann algebra $\mathbb{M}_{G}$ is completely determined by the cardinalities $\left\{\left|\sum_{n}^{(N)}\right|\right\}_{n=1}^{\infty}$ of certain sets $\left\{\sum_{n}^{(N)}\right\}_{n=1}^{\infty}$, where

$$
\sum_{n}^{(N)}=\left\{\left(t_{i_{1}}, \ldots, t_{i_{n}}\right) \in( \pm X)^{n}: \sum_{j=1}^{n} t_{i_{j}}=x_{0}\right\}
$$

where

$$
t_{i_{j}} \stackrel{\text { def }}{=} \begin{cases}x_{i_{j}} & \text { if } t_{i_{j}} \in X \\ -x_{i_{j}} & \text { if } t_{i_{j}} \in-X\end{cases}
$$

for all $j=1, \ldots, n$, for all $n \in \mathbb{N}$, where $X=\left\{x_{1}, \ldots, x_{N}\right\} \subset \mathbb{C}^{\oplus N}$ is the labeling set of $G$ consisting of the orthonormal vectors $x_{j}=(0, \ldots, 0, \underset{j \text {-th }}{1}, 0, \ldots, 0)$, for $j=$ $1, \ldots, n$, and where

$$
x_{0}=(\underbrace{0, \ldots \ldots \ldots, 0}_{N \text {-times }}) \in \mathbb{C}^{\oplus N},
$$

where

$$
N=\max \left\{\operatorname{deg}_{\text {out }}(v): v \in V(G)\right\}
$$

By using the operation $\theta$, defined in Section 3.2, we can re-write $\sum_{n}^{(N)}$ 's by

$$
\sum_{n}^{(N)}=\left\{w \in( \pm X)^{n}: \theta(w)=x_{0}\right\}
$$

Construct now the lattice paths in $\mathbb{R}^{2}$.

Definition 5.2. Let $Y=\{1, \ldots, N\}$, for $N \in \mathbb{N}$. For the given index set $Y$, define the vectors $l_{ \pm k}$ by the vectors in $\mathbb{R}^{2}$ satisfying that 


$$
l_{k}=\left(1, e^{k}\right) \text { and } l_{-k}=\left(1,-e^{k}\right), \text { for all } k=1, \ldots, N,
$$

where $e \in \mathbb{R}$ is the natural (exponential) number. i.e., $l_{k}$ (resp. $l_{-k}$ ) is a vector starting from the point $(0,0)$ and ending at the point $\left(1, e^{k}\right)$ (resp. the point $(1$, $\left.-e^{k}\right)$ ), for all $k=1, \ldots, N$. Define now the binary operation, denoted simply by $(\cdot)$, on $\left\{l_{ \pm k}: k=1, \ldots, N\right\}$, by

$l_{i} l_{j} \stackrel{\text { def }}{=}$ the path in $\mathbb{R}^{2}$ connecting the vector $l_{i}$ and $l_{j}$, by identifying the ending point $\left(1, e^{i}\right)$ of $l_{i}$ and the starting point $(0,0)$ of $l_{j}$,

where $i, j \in\{ \pm 1, \ldots, \pm N\}$. Inductively, we can determine the paths $l_{i_{1}} l_{i_{2}} \ldots$ $l_{i_{n}}$, where $\left(i_{1}, \ldots, i_{n}\right) \in\{ \pm 1, \ldots, \pm N\}^{n}$, for all $n \in \mathbb{N}$. Such paths in $\mathbb{R}^{2}$, generated by the vectors $l_{ \pm 1}, \ldots, l_{ \pm N}$, are called the lattice paths generated by $Y$. We denote the collection of all lattice paths generated by the vectors $l_{ \pm 1}, \ldots, l_{ \pm N}$, by $\mathcal{L}_{N}$. And we call $\mathcal{L}_{N}$, the lattice path set induced by $Y$.

Let $l=l_{i_{1}} \ldots l_{i_{n}} \in \mathcal{L}_{N}$ be a lattice path. Define the length of $l$ by the number $n$. And we denote the length of $l$ by $|l|$.

Notice that every element in the lattice path set $\mathcal{L}_{N}$ is a (non-reduced and nonempty) words in $\left\{l_{ \pm 1}, \ldots, l_{ \pm N}\right\}$. We can decompose $\mathcal{L}_{N}$ by

$$
\mathcal{L}_{N}={\stackrel{\sqcup}{~} \mathcal{L}^{\infty}}_{\mathcal{L}_{N}}(k)
$$

with

$$
\mathcal{L}_{N}(k) \stackrel{\text { def }}{=}\left\{l \in \mathcal{L}_{N}:|l|=k\right\}, \text { for all } k \in \mathbb{N},
$$

where "ப" means the disjoint union.

Remark 5.2. Remark that, we define the lattices $l_{ \pm k}$ by the vectors $\left(1, \pm e^{k}\right)$, for $k=1, \ldots, N$. The choice of the $y$-coordinates $\pm e^{k}$ (by regarding the space $\mathbb{R}^{2}$ as the usual $(x, y)$-coordinate system) is for our purpose, for $k=1, \ldots, N$. i.e., we want to make the sets $\mathcal{L}_{N}(k)$ be equipotent (or bijective) to the sets $\sum_{k}^{(N)}$, for each $k=1, \ldots, N$, where $\sum_{k}^{(N)}$ are the given subsets of $\mathbb{C}^{\oplus N}$, in the previous section. It is okay to take $\pi$ instead of $e$.

Consider a certain subset $\mathcal{L}_{N}^{o}(k)$ of $\mathcal{L}_{N}(k)$, for each $k \in \mathbb{N}$ : Define the subset $\mathcal{L}_{N}^{o}(k)$ of $\mathcal{L}_{N}(k)$ by

$$
\mathcal{L}_{N}^{o}(k) \stackrel{\text { def }}{=}\left\{\begin{array}{l|l}
l \in \mathcal{L}_{N}(k): & \begin{array}{c}
l \text { ends at the point on } \\
\text { the horizontal axis } \\
\text { (or the } x \text {-axis) of } \mathbb{R}^{2}
\end{array}
\end{array}\right\} .
$$


i.e., if $l=l_{i_{1}} \ldots l_{i_{n}} \in \mathcal{L}_{N}^{o}(k)$, then it is a lattice path starting from $(0,0)$, ending at $(k, 0)$.

Definition 5.3. If a lattice path $l$ is contained in $\mathcal{L}_{N}^{o}(k)$, for some $k \in \mathbb{N}$, then we say that the length-k lattice path $l$ satisfies the (horizontal- or $x$-)axis property.

Similarly, we can define the subset $\mathcal{L}_{N}^{o}$ of the lattice path set $\mathcal{L}_{N}$ by

$$
\mathcal{L}_{N}^{o} \stackrel{\text { def }}{=} \bigcup_{k=1}^{\infty} \mathcal{L}_{N}^{o}(k)
$$

i.e., the subset $\mathcal{L}_{N}^{o}$ is the collection of all lattice paths satisfying the axis property.

By defining a map $\Theta: \sum_{n}^{(N)} \rightarrow \mathcal{L}_{N}^{o}(n)$, we can realize the relation between the sets $\sum_{n}^{(N)}$ and $\mathcal{L}_{N}^{o}(n)$, for all $n \in \mathbb{N}$. Define the map $\Theta$ by

where

$$
\left(t_{i_{1}}, \ldots, t_{i_{n}}\right) \in \sum_{n}^{(N)} \stackrel{\Theta}{\longrightarrow} l_{k_{1}} \ldots l_{k_{n}} \in \mathcal{L}_{N}^{o}(n),
$$

$$
k_{j}= \begin{cases}i_{j} & \text { if } t_{i_{j}}=x_{i_{j}} \in X \\ -i_{j} & \text { if } t_{i_{j}}=-x_{i_{j}} \in-X\end{cases}
$$

for all $j=1, \ldots, n$, for all $n \in \mathbb{N}$. By the very definition of the map $\Theta$, this map is bijective. Therefore, we can realize that the property

$$
\theta\left(\left(t_{i_{1}}, \ldots, t_{i_{n}}\right)\right)=\sum_{j=1}^{n} t_{i_{j}}=x_{0} \in \mathbb{C}^{\oplus N}
$$

is equivalent to the axis property: the lattice path

$$
\Theta\left(\left(t_{i_{1}}, \ldots, t_{i_{n}}\right)\right)=l_{k_{1}} \ldots l_{k_{n}} \in \mathcal{L}_{N}^{o}(n)
$$

satisfies the axis property. By the existence of the above bijection $\Theta$, we have the following proposition.

Proposition 5.8. The sets $\sum_{n}^{(N)}$ and $\mathcal{L}_{N}^{o}(n)$ are equipotent (or bijective), for all $n \in \mathbb{N}$. i.e.,

$$
\left|\sum_{n}^{(N)}\right|=\left|\mathcal{L}_{N}^{o}(n)\right|, \text { for all } n \in \mathbb{N}
$$

The following corollary is the direct consequence of the previous proposition. 
Corollary 5.9. Let $G$ be a canonical weighted graph with its labeled graph groupoid $\mathbb{G}$, and assume that $\mathbb{G}$ is a fractaloid. Then the labeling operator $T_{G}$ of $\mathbb{G}$ in $\mathbb{M}_{G}$ has its $\mathbb{D}_{G}$-valued moments,

$$
E\left(T_{G}^{n}\right)=\left|\mathcal{L}_{N}^{o}(n)\right| \cdot 1_{\mathbb{D}_{G}}
$$

for all $n \in \mathbb{N}$.

In fact, the length- $n$ lattice path set $\mathcal{L}_{N}^{o}(n)$ satisfying the axis property is introduced for visualizing the set $\sum_{n}^{(N)}$, for all $n \in \mathbb{N}$. By identifying (bijectively) the sets $\left\{\sum_{n}^{(N)}\right\}_{n=1}^{\infty}$ and $\left\{\mathcal{L}_{N}^{o}(n)\right\}_{n=1}^{\infty}$, we can get the following proposition.

Proposition 5.10. Let $T_{G}$ be the labeling operator of a fractaloid $\mathbb{G}$ in $\mathbb{M}_{G}$. Then all odd $\mathbb{D}_{G}$-valued moments vanish. i.e., $E\left(T_{G}^{n}\right)=0_{\mathbb{D}_{G}}$, whenever $n$ is odd.

Proof. We can easily verify that if $n$ is odd, then the set $\mathcal{L}_{N}^{o}(n)$ is empty. So, if $n$ is odd, then $\left|\mathcal{L}_{N}^{o}(n)\right|=0$. So, we have that

$$
E\left(T_{G}^{n}\right)=\left|\sum_{n}^{(N)}\right| \cdot 1_{\mathbb{D}_{G}}=\left|\mathcal{L}_{N}^{o}(n)\right| \cdot 1_{\mathbb{D}_{G}}=0_{\mathbb{D}_{G}},
$$

whenever $n$ is odd in $\mathbb{N}$.

By the previous proposition, we can get the more detailed $\mathbb{D}_{G}$-free distributional data of the labeling operator $T_{G}$ of the fractaloid $\mathbb{G}$ :

Corollary 5.11. Let $T_{G}$ be the labeling operator of a fractaloid $\mathbb{G}$ in the right graph von Neumann algebra $\mathbb{M}_{G}$. Then

$$
E\left(T_{G}^{n}\right)= \begin{cases}\left|\sum_{n}^{(N)}\right| \cdot 1_{\mathbb{D}_{G}}=\left|\mathcal{L}_{N}^{o}(n)\right| \cdot 1_{\mathbb{D}_{G}} & \text { if } n \in 2 \mathbb{N} \\ 0_{\mathbb{D}_{G}} & \text { if } n \in 2 \mathbb{N}-1,\end{cases}
$$

for all $n \in \mathbb{N}$.

Now, we concentrate on finding the cardinality $\left|\mathcal{L}_{N}^{o}(2 n)\right|$, for $n \in \mathbb{N}$. To do that, we consider the results in [38] and [39]. In [38], we compute the powers of multinomials in a commutative unital algebra. We showed that the computations are determined by the well-known famous Pascal's triangle, recursively.

Let $A$ be a commutative unital algebra with its identity $1_{A}$, and let $a_{1}, \ldots, a_{n}$ be arbitrary distinct elements in $A$, for $n \in \mathbb{N}$. Then we can construct a new element 
$a=\sum_{k=1}^{n} a_{j}$ in $A$, and the powers $a^{m}$ of $a$ in $A$, for all $m \in \mathbb{N}$. We will call the element $a$ the multinomial induced by $a_{1}, \ldots, a_{n}$, and the elements $a^{m}$, the $m$-th powers of $a$, for all $m \in \mathbb{N}$. Then, by the commutativity on $A$, we can get that

$$
\begin{aligned}
a^{m} & =\sum_{\left(i_{1}, \ldots, i_{m}\right) \in\{1, \ldots, n\}^{m}} a_{i_{1}} \ldots a_{i_{m}} \\
& =\sum_{\left(i_{1}, \ldots, i_{m}\right) \in\{1, \ldots, n\}^{m}, i_{1} \leq i_{2} \leq \ldots \leq i_{m}} c_{i_{1}, \ldots, i_{m}}^{(n)} a_{i_{1}} \ldots a_{i_{m}},
\end{aligned}
$$

where $c_{i_{1}, \ldots, i_{m}}^{(n)} \in \mathbb{N}$ are called the $\left(i_{1}, \ldots, i_{m}\right)$-th coefficients of $a_{i_{1}} \ldots a_{i_{m}}$ in $a^{m}$, for $\left(i_{1}, \ldots, i_{m}\right) \in\{1, \ldots, n\}^{m}$, where $i_{1} \leq i_{2} \leq \ldots \leq i_{m}$. The existence of the coefficients of $a^{m}$ is guaranteed by the commutativity of $A$, for all $m \in \mathbb{N}$. It is natural that if we know how to compute all coefficients of $a^{m}$, then we can compute $a^{m}$.

For convenience, let's denote $1_{A}$ be $a_{0}$. Fix $a_{j} \in\left\{a_{1}, \ldots, a_{n}\right\}$. Then we can consider the binomial $y_{j}=1_{A}+a_{j}=a_{0}+a_{j}$ in $A$, for all $j=1, \ldots, n$. Then the powers $y_{j}^{m}$ have their coefficients completely determined by the Pascal's triangle, for all $j=1, \ldots, n$, and $m \in \mathbb{N}$. i.e., we can write

$$
y_{j}^{m}=\sum_{\left(i_{1}, \ldots, i_{m}\right) \in\{0, j\}^{m}, i_{1} \leq i_{2} \leq \ldots \leq i_{m}} \varepsilon_{j: i_{1}, \ldots, i_{m}} a_{i_{1}} \ldots a_{i_{m}}
$$

where $\varepsilon_{j: i_{1}, \ldots, i_{m}}$ 's are the $\left(i_{1}, \ldots, i_{m}\right)$-th coefficients of $y_{j}^{m}$, for all $j=1, \ldots, n$, and $m \in \mathbb{N}$, and they are the entries of the Pascal's triangle,

$\begin{array}{cccccccccccc} & & & & & 1 & & & & & \\ & & & & 1 & & 1 & & & & \\ & & & 1 & & 2 & & 1 & & & \\ & & 1 & & 3 & & 3 & & 1 & & \\ & 1 & & 4 & & 6 & & 4 & & 1 & \\ 1 & & 5 & & 10 & & 10 & & 5 & & 1 \\ & & & & & & & & & \\ \vdots & & \vdots & & \vdots & & \vdots & & \vdots & & \vdots\end{array}$

In other words, the entry $\varepsilon_{j:} \underbrace{0, \ldots \ldots \ldots, 0}_{k \text {-times }}, \underbrace{j, \ldots \ldots \ldots ., j}_{(m-k) \text {-times }}$ are the $(m-k)$-th entry (from the left) of the $m$-th level of the Pascal's triangle. Equivalently, the above triangle can be re-written as follows, in terms of the coefficients of $y_{j}^{m}$ : 


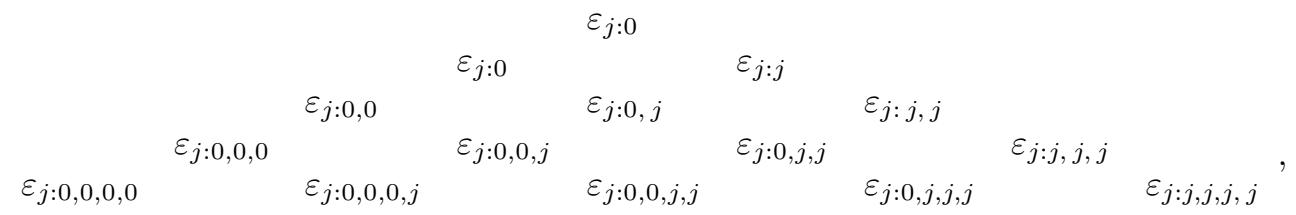

$$
\begin{aligned}
& \begin{array}{lll} 
& \vdots & \vdots
\end{array}
\end{aligned}
$$

for all $j=1, \ldots, n$, and for $m \in \mathbb{N}$. The following proposition is the main result of [38]:

Proposition 5.12. (See [38]) Let $a=\sum_{j=1}^{n} a_{j}$ be a multinomial induced by the distinct elements $a_{1}, \ldots, a_{n}$ in a commutative unital algebra $A$, for $n \in \mathbb{N}$. Then the powers $a^{m}$ of a has the coefficients $c_{i_{1}, \ldots, i_{m}}^{(n)}$, satisfying that

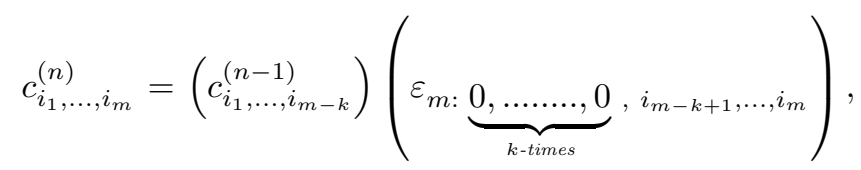

for $k \in\{1, \ldots, m\}$, for all $m \in \mathbb{N}$, whenever

$$
i_{m-k+1}=i_{m-k+2}=\ldots=i_{m} \text { in }\{1, \ldots, n\} .
$$

The proof of the above proposition is straightforward. The above proposition shows that the coefficients of the $m$-th power $a^{m}$ of a multinomial $a=\sum_{j=1}^{n} a_{j}$ are determined by the entries of the Pascal's triangle, recursively. The following three examples are helpful to understand the use of the above proposition.

Example 5.1. The coefficient $c_{1,1,1,1,3,3,4}^{(4)}$ of $a_{1}^{4} a_{3}^{2} a_{4}$ in $\left(\sum_{k=1}^{4} a_{k}\right)^{7}$ is

$$
\begin{aligned}
c_{1,1,1,1,3,3,4}^{(4)} & =c_{1,1,1,1,3,3}^{(3)} \varepsilon_{4: 0,0,0,0,0,0,4}=7 \cdot c_{1,1,1,1,3,3}^{(3)} \\
& =7 \cdot c_{1,1,1,1}^{(2)} \varepsilon_{3: 0,0,0,0,3,3}=7 \cdot 15 \cdot 1=105
\end{aligned}
$$

Example 5.2. The coefficient $c_{2,2,3,4}^{(5)}$ of $a_{2}^{2} a_{3} a_{4}$ in $\left(\sum_{k=1}^{5} a_{k}\right)^{4}$ is

$$
\begin{aligned}
c_{2,2,3,4}^{(5)} & =c_{2,2,3}^{(4)} \cdot \varepsilon_{5: 0,0,0,4}=4 \cdot c_{2,2,3}^{(4)} \\
& =4 \cdot c_{2,2}^{(3)} \cdot \varepsilon_{4: 0,0,3}=4 \cdot 1 \cdot 3=12
\end{aligned}
$$


Example 5.3. The coefficient $c_{1,2,3,4}^{(4)}$ of $a_{1} a_{2} a_{3} a_{4}$ in $\left(\sum_{k=1}^{4} a_{k}\right)^{4}$ is

$$
\begin{aligned}
c_{1,2,3,4}^{(4)} & =c_{1,2,3}^{(3)} \cdot \varepsilon_{4: 0,0,0,4}=4 \cdot c_{1,2,3}^{(3)} \\
& =4 \cdot c_{1,2}^{(2)} \cdot \varepsilon_{3: 0,0,3}=4 \cdot 3 \cdot c_{1,2}^{(2)}=4 \cdot 3 \cdot 2=24 .
\end{aligned}
$$

Now, consider the lattice paths in $\mathcal{L}_{N}(2 n)$. Every lattice path $l$ contained in $\mathcal{L}_{N}(2 n)$ is the length- $2 n$ (non-reduced and nonempty) word in $\left\{l_{ \pm 1}, \ldots, l_{ \pm N}\right\}$, say $l=l_{j_{1}} l_{j_{2}} \ldots l_{j_{2 n}}$. As we observed before, we can realize that this lattice $l$ satisfies the axis property if and only if

$$
\sum_{k=1}^{2 n} j_{k}=0
$$

Assume that we have distinct elements $a_{1}, a_{-1}, a_{2}, a_{-2}, \ldots, a_{N}, a_{-N}$ in a commutative unital algebra $A$. Then we can have an element

$$
a_{ \pm}=\sum_{k=-N}^{-1} a_{k}+\sum_{i=1}^{N} a_{i}, \text { in } A .
$$

Then the coefficients, denoted by $c_{i_{1}, \ldots, i_{2 n}}^{( \pm N)}$ of the $2 n$-th power $a_{ \pm}^{2 n}$ of $a_{ \pm}$are also determined similarly by the above proposition, for all $\left(i_{1}, \ldots, i_{2 n}\right) \in\{ \pm 1, \ldots$, $\pm N\}^{2 n}$, for $n \in \mathbb{N}$. By the previous observation, we can realize that the addition of all coefficients

$$
c_{i_{1}, \ldots, i_{2 n}}^{( \pm N)} \text { satisfying that } \sum_{k=1}^{2 n} i_{k}=0,
$$

represents the cardinalities of the length- $2 n$ lattice paths satisfying the axis property. Therefore, we can get the following theorem.

Theorem 5.13. Let $\mathcal{L}_{N}^{o}(2 n)$ be the set of all length-2n lattice paths induced by $l_{ \pm 1}$, $\ldots, l_{ \pm N}$, for any $N, n \in \mathbb{N}$. And assume that $c_{i_{1}, \ldots, i_{2 n}}^{( \pm N)}$ are the coefficients of the $2 n$-th power $a_{ \pm}^{2 n}$, for $n \in \mathbb{N}$, where $a_{ \pm}$is defined as in the previous paragraph. Then

$$
\left|\mathcal{L}_{N}^{o}(2 n)\right|=\sum_{\left(j_{1}, \ldots, j_{2 n}\right) \in\{ \pm 1, \ldots, \pm N\}^{2 n}, \sum_{k=1}^{2 n} j_{k}=0} c_{j_{1}, \ldots, j_{2 n}}^{( \pm N)},
$$

for all $n \in \mathbb{N}$. 
The above theorem shows that the amalgamated moments of the labeling operator of fractaloids are also determined by certain entries of the Pascal's triangle, recursively.

Corollary 5.14. Let $c_{i_{1} \ldots, i_{n}}^{( \pm N)}$ be the coefficients of the $2 n$-power $a_{ \pm}^{2 n}$ of $a_{ \pm}$, defined as above. And let $T_{G}$ be the labeling operator of a fractaloid $\mathbb{G}$ in the right graph von Neumann algebra $\mathbb{M}_{G}$, where $N=\max \left\{\operatorname{deg}_{\text {out }}(v): v \in V(G)\right\}$. Then the nonzero $\mathbb{D}_{G}$-valued moments $\left\{E\left(T_{G}^{2 n}\right)\right\}_{n=1}^{\infty}$ of $T_{G}$ are determined by

$$
E\left(T_{G}^{2 n}\right)=\left(\sum_{\left(j_{1}, \ldots, j_{2 n}\right) \in\{ \pm 1, \ldots, \pm N\}^{2 n}, \sum_{k=1}^{2 n} j_{k}=0} c_{j_{1}, \ldots, j_{2 n}}^{( \pm N)}\right) \cdot 1_{\mathbb{D}_{G}}
$$

for all $n \in \mathbb{N}$.

\subsection{An Example: Computing $\left|\mathcal{L}_{1}^{o}(2 n)\right|=\left|\sum_{2 n}^{(1)}\right|$, for $n \in \mathbb{N}$.}

In this section, we will provide an example to use the lattice path model $\left\{\mathcal{L}_{N}^{o}(n)\right\}_{n=1}^{\infty}$ to compute $\left\{\left|\sum_{n}^{(N)}\right|\right\}_{n=1}^{\infty}$. Again, remark that

$$
E\left(T_{G}^{n}\right)=\left|\sum_{n}^{(N)}\right| \cdot 1_{D_{G}}, \text { for all } n \in \mathbb{N},
$$

and the sets $\sum_{n}^{(N)}$ are visualized by the length- $n$ lattice paths contained in $\mathcal{L}_{N}^{o}(n)$ satisfying the axis property, for all $n \in \mathbb{N}$, where

$$
N=\max \left\{\operatorname{deg}_{\text {out }}(v): v \in V(G)\right\} .
$$

In the rest of this section, as an example of Section 5.4, we will compute

$$
\left|\mathcal{L}_{1}^{o}(2 n)\right|=\left|\sum_{2 n}^{(1)}\right|, \text { for all } n \in \mathbb{N}
$$

(Case: $n=2$ ) If $n=2$, then we have the following elements of $\mathcal{L}_{1}^{o}(2)$ :

And hence

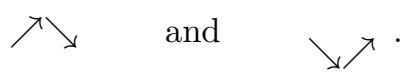

$$
\left|\mathcal{L}_{1}^{o}(2)\right|=\left|\sum_{2}^{(1)}\right|=2
$$

(Case: $n=4$ ) If $n=4$, then $\mathcal{L}_{1}^{o}(4)$ has the following elements: 
and

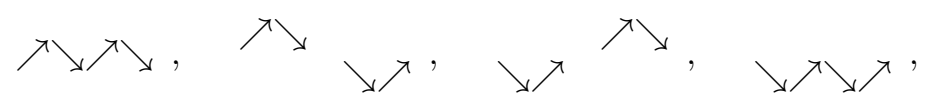

So, we have

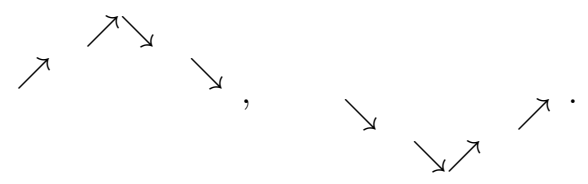

$$
\left|\mathcal{L}_{1}^{o}(4)\right|=\left|\sum_{4}^{(1)}\right|=6
$$

(Case: $n=6$ ) If $n=6$, then $\mathcal{L}_{1}^{o}(6)$ has the following elements:

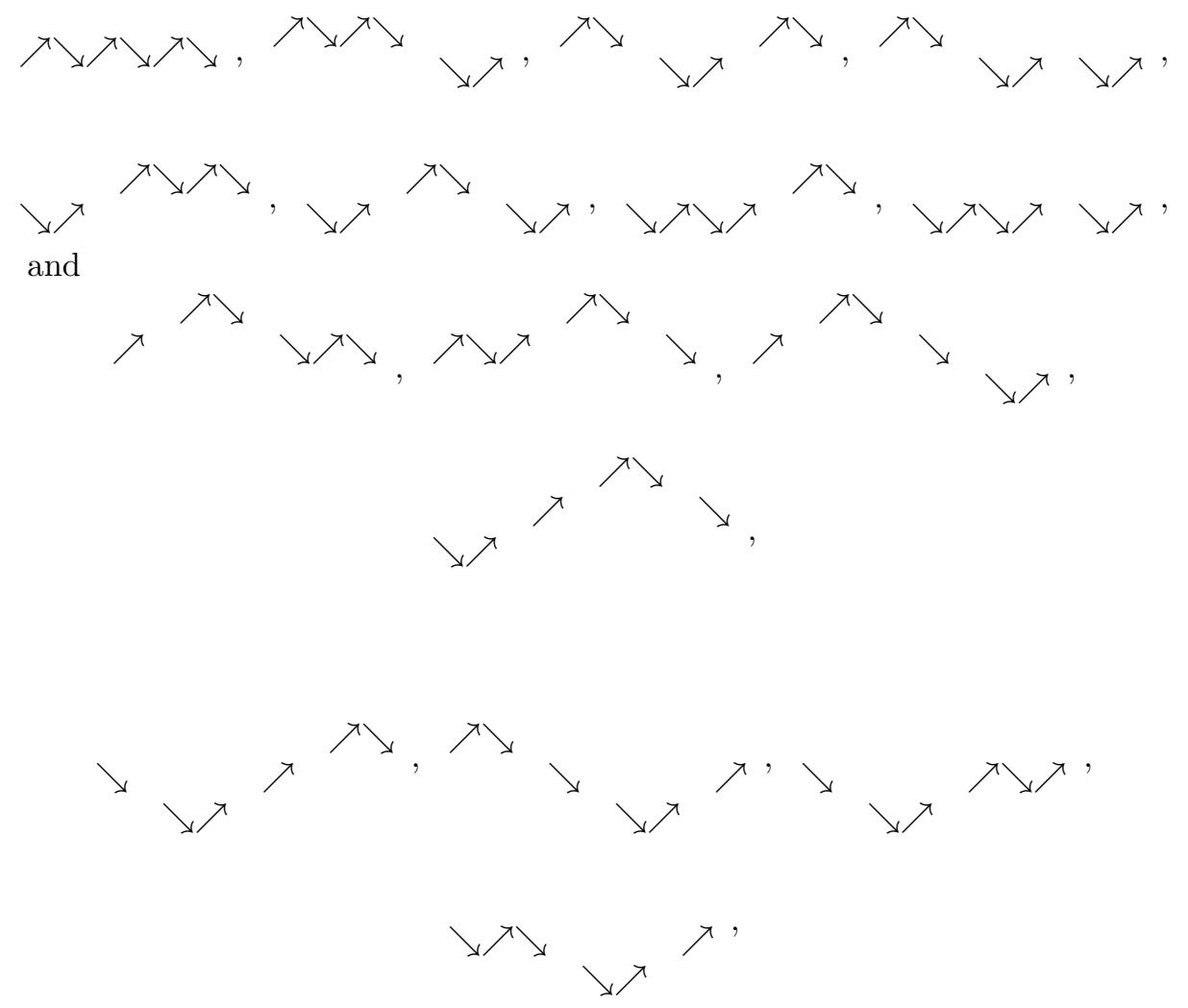

and

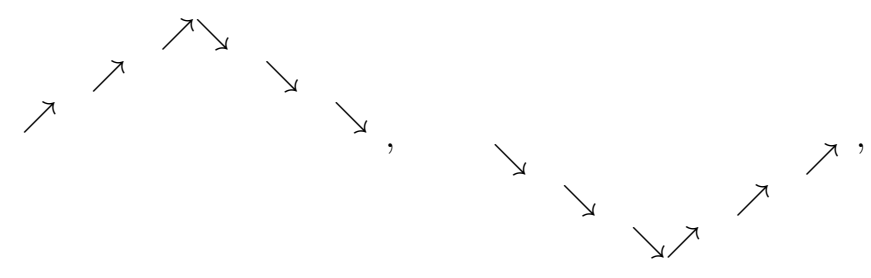




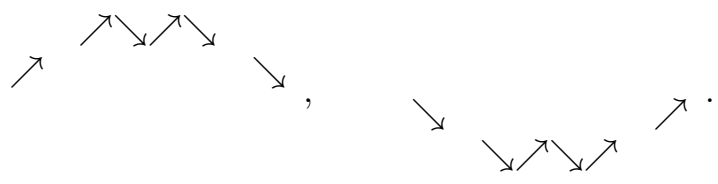

Therefore, we can get that

$$
\left|\mathcal{L}_{1}^{o}(6)\right|=\left|\sum_{6}^{(1)}\right|=20,
$$

etc.

The above visual observations for $n=2,4,6$, provide a way how to compute $\left|\mathcal{L}_{1}^{o}(n)\right|$, for $n=2,4,6$. However, in general, if $n$ is an even number greater than 6 , the above observation is extremely hard. But, by using the technique determined recursively by the Pascal's triangle, we can compute $\left|\mathcal{L}_{1}^{o}(2 n)\right|$, for all $n \in \mathbb{N}$.

As we have seen, we can realize that the cardinalities of the set $\mathcal{L}_{1}^{o}(2 k)$, for $k=$ $1,2,3$, are indeed determined by the certain entries of the Pascal's triangle,

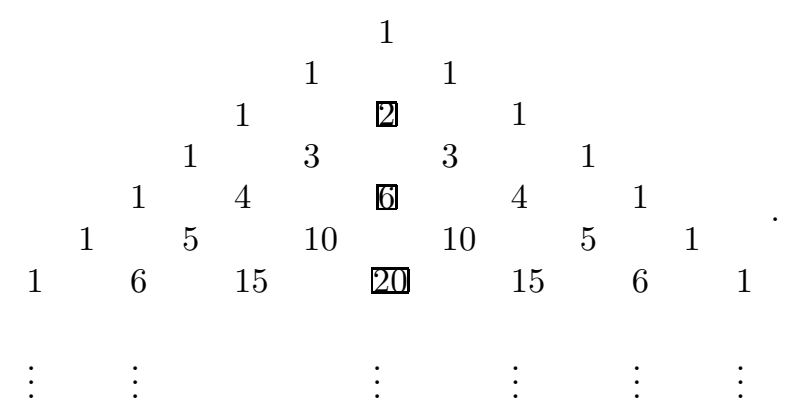

Recall that, by Section 5.4, we can have that:

Corollary 5.15. For any $n \in \mathbb{N}$,

$$
\left|\mathcal{L}_{1}^{o}(2 n)\right|=c_{n \text {-times }}^{( \pm 1)} \underbrace{(1, \ldots \ldots \ldots,-1}_{n \text {-times }}, \underbrace{1, \ldots \ldots \ldots \ldots, 1}=\left|\sum_{2 n}^{(1)}\right|,
$$

where $c_{-1, \ldots,-1,1, \ldots, 1}^{( \pm 1)}$ are the $(-1, \ldots,-1,1, \ldots, 1)$-th coefficients of the $2 n$-power $\left(a_{-1}+a_{1}\right)^{2 n}$ of the binomial $a_{-1}+a_{1}$, for all $n \in \mathbb{N}$. Equivalently, we have that

$$
\left|\mathcal{L}_{1}^{o}(2 n)\right|={ }_{2 n} C_{n}=\left|\sum_{2 n}^{(1)}\right|, \text { for all } n \in \mathbb{N}
$$

where ${ }_{m} C_{k} \stackrel{\text { def }}{=} \frac{m !}{k !(m-k) !}$, for all $k \leq n$ in $\mathbb{N}$. 
Indeed, the coefficients in the above corollary represents the middle entries of $2 n$-levels of the Pascal's triangle, for all $n \in \mathbb{N}$.

Example 5.4. $\left|\mathcal{L}_{1}^{o}(8)\right|=70=\left|\sum_{8}^{(1)}\right|, \quad\left|\mathcal{L}_{1}^{o}(10)\right|=252=\left|\sum_{10}^{(1)}\right|$,

$$
\begin{aligned}
& \left|\mathcal{L}_{1}^{o}(12)\right|=924=\left|\sum_{12}^{(1)}\right|, \quad\left|\mathcal{L}_{1}^{o}(14)\right|=3432=\left|\sum_{14}^{(1)}\right| \\
& \left|\mathcal{L}_{1}^{o}(16)\right|=12870=\left|\sum_{16}^{(1)}\right|,\left|\mathcal{L}_{1}^{o}(18)\right|=48620=\left|\sum_{18}^{(1)}\right| \\
& \left|\mathcal{L}_{1}^{o}(20)\right|=184756=\left|\sum_{20}^{(1)}\right|, \text { etc. }
\end{aligned}
$$

\section{References}

[1] A. G. Myasnikov and V. Shapilrain (editors), Group Theory, Statistics and Cryptography, Contemporary Math, 360, (2003) AMS.

[2] A. Gibbons and L. Novak, Hybrid Graph Theory and Network Analysis, ISBN: 0-521-46117-0, (1999) Cambridge Univ. Press.

[3] B. Solel, You can see the arrows in a Quiver Operator Algebras, (2000), preprint.

[4] C. W. Marshall, Applied Graph Theory, ISBN: 0-471-57300-0 (1971) John Wiley \& Sons

[5] D.Voiculescu, K. Dykemma and A. Nica, Free Random Variables, CRM Monograph Series Vol 1 (1992).

[6] D.W. Kribs and M.T. Jury, Ideal Structure in Free Semigroupoid Algebras from Directed Graphs, preprint.

[7] D.W. Kribs, Quantum Causal Histories and the Directed Graph Operator Framework, arXiv:math.OA/0501087v1 (2005), Preprint.

[8] F. Balacheff, Volume Entropy, Systole and Stable Norm on Graphs, arXiv:math.MG/0411578v1, (2004) Preprint.

[9] G. C. Bell, Growth of the Asymptotic Dimension Function for Groups, (2005) Preprint.

[10] I. Cho, Graph von Neumann algebras, ACTA. Appl. Math, 95, (2007) $95-135$.

[11] I. Cho, Characterization of Free Blocks of a right graph von Neumann algebra, Compl. An. \& Op. theo (2007) To be Appeared.

[12] I. Cho, Direct Producted $W^{*}$-Probability Spaces and Corresponding Free Stochastic Integration, B. of KMS, 44, No. 1, (2007), 131 - 150. 
[13] I. Cho, Vertex-Compressed Algebras of a Graph von Neumann Algebra, (2007) Submitted to ACTA. Appl. Math.

[14] I. Cho, Group-Freeness and Certain Amalgamated Freeness, J. of KMS, 45, no. 3, (2008) 597 - 609.

[15] I. Cho and Palle Jorgensen, $C^{*}$-Algebras Generated by Partial Isometries, JAMC, (2008) To Appear.

[16] I. Raeburn, Graph Algebras, CBMS no 3, AMS (2005).

[17] P. D. Mitchener, $C^{*}$-Categories, Groupoid Actions, Equivalent KK-Theory, and the Baum-Connes Conjecture, arXiv:math.KT/0204291 1 , (2005), Preprint.

[18] R. Scapellato and J. Lauri, Topics in Graph Automorphisms and Reconstruction, London Math. Soc., Student Text 54, (2003) Cambridge Univ. Press.

[19] R. Exel, A new Look at the Crossed-Product of a $C^{*}$-algebra by a Semigroup of Endomorphisms, (2005) Preprint.

[20] R. Gliman, V. Shpilrain and A. G. Myasnikov (editors), Computational and Statistical Group Theory, Contemporary Math, 298, (2001) AMS.

[21] R. Speicher, Combinatorial Theory of the Free Product with Amalgamation and Operator-Valued Free Probability Theory, AMS Mem, Vol 132 , Num 627 , (1998).

[22] S. H. Weintraub, Representation Theory of Finite Groups: Algebra and Arithmetic, Grad. Studies in Math, vo. 59, (2003) AMS.

[23] V. Vega, Finite Directed Graphs and $W^{*}$-Correspondences, (2007) $\mathrm{Ph}$. D thesis, Univ. of Iowa.

[24] W. Dicks and E. Ventura, The Group Fixed by a Family of Injective Endomorphisms of a Free Group, Contemp. Math 195, AMS.

[25] F. Radulescu, Random Matrices, Amalgamated Free Products and Subfactors of the von Neumann Algebra of a Free Group, of Noninteger Index, Invent. Math., 115, (1994) 347 - 389.

[26] D. A. Lind, Entropies of Automorphisms of a Topological Markov Shift, Proc. AMS, vo 99, no 3, (1987) 589 - 595.

[27] D. A. Lind and B. Marcus, An Introduction to Symbolic Dynamics and Coding, (1995) Cambridge Univ. Press.

[28] D. A. Lind and S. Tuncel, A Spanning Tree Invariant for Markov Shifts, IMA Vol. Math. Appl., vo 123, (2001), 487 - 497.

[29] D. A. Lind and K. Schmidt, Symbolic and Algebraic Dynamical Systems, Handbook of Dynamical System, Vol.\1A, (2002) 765 - 812.

[30] R. V. Kadison and J. R. Ringrose, Fundamentals of the Theory of Operator Algebra, Grad. Stud. Math., vo. 15, (1997) AMS.

[31] D. E. Dutkay and P. E. T. Jorgensen, Iterated Function Systems, Ruelle Operators and Invariant Projective Measures, arXiv:math.DS/0501077/v3, (2005) Preprint.

[32] P. E. T. Jorgensen, Use of Operator Algebras in the Analysis of Measures from Wavelets and Iterated Function Systems, (2005) Preprint.

[33] D. Guido, T. Isola and M. L. Lapidus, A Trace on Fractal Graphs and the Ihara Zeta Function, arXiv:math.OA/0608060v1, (2006) Preprint. 
[34] P. Potgieter, Nonstandard Analysis, Fractal Properties and Brownian Motion, arXiv:math.FA/0701649v1, (2007) Preprint.

[35] L. Bartholdi, R. Grigorchuk, and V. Nekrashevych, From Fractal Groups to Fractal Sets, arXiv:math.GR/0202001v4, (2002) Preprint.

[36] I. Cho, The Moments of Certain Perturbed Operators of the Radial Operator of the Free Group Factor $L\left(F_{N}\right)$, JAA, 5, no. 3, (2007) $137-165$.

[37] I. Cho and P. E. T. Jorgensen, $C^{*}$-Subalgebras Generated by Partial Isometries in $B(H)$, (2007) Submitted to JMP.

[38] S. Thompson and I. Cho, Powers of Mutinomials in Commutative Algebras, (2008) (Undergraduate Research) Submitted to PMEJ.

[39] S. Thompson, C. M. Mendoza, and A. J. Kwiatkowski, and I. Cho, Lattice Paths Satisfying the Axis Property, (2008) (Undergraduate Research) Preprint.

[40] I. Cho, Labeling Operators of Graph Groupoids, (2008) Preprint.

[41] R. T. Powers, Heisenberg Model and a Random Walk on the Permutation Group, Lett. Math. Phys., 1, no. 2, (1975) 125 - 130.

[42] R. T. Powers, Resistance Inequalities for $K M S$-states of the isotropic Heisenberg Model, Comm. Math. Phys., 51, no. 2, (1976) 151 $-156$.

[43] R. T. Powers, Registance Inequalities for the Isotropic Heisenberg Ferromagnet, JMP, 17, no. 10, (1976) 1910 - 1918.

[44] E. P. Wigner, Characteristic Vectors of Bordered Matrices with Infinite Dimensions, Ann. of Math. (2), 62, (1955) 548 - 564.

[45] D. Voiculescu, Symmetries of Some Reduced Free Product $C^{*}$ Algebras, Lect. Notes in Math., 1132, Springer, (1985) 556 - 588.

[46] T. Shirai, The Spectrum of Infinite Regular Line Graphs, Trans. AMS., 352, no 1., (2000) 115 - 132.

[47] J. Kigami, R. S. Strichartz, and K. C. Walker, Constructing a Laplacian on the Diamond Fractal, Experiment. Math., 10, no. 3, (2001) $437-448$.

[48] I. V. Kucherenko, On the Structurization of a Class of Reversible Cellular Automata, Diskret. Mat., 19, no. 3, (2007) 102 - 121.

[49] J. L. Schiff, Cellular Automata, Discrete View of the World, Wiley-Interscience Series in Disc. Math .\& Optimazation, ISBN: 9780-470-16879-0, (2008) John Wiley \& Sons Press.

[50] P. E. T. Jorgensen, and M. Song, Entropy Encoding, Hilbert Spaces, and Kahunen-Loeve Transforms, JMP, 48, no. 10, (2007)

[51] P. E. T. Jorgensen, L. M. Schmitt, and R. F. Werner, q-Canonical Commutation Relations and Stability of the Cuntz Algebra, Pac. J. of Math., 165, no. 1, (1994) 131 - 151.

[52] A. Gill, Introduction to the Theory of Finite-State Machines, MR0209083 (34\\#8891), (1962) McGraw-Hill Book Co.

[53] J. E. Hopcroft, and J. D. Ullman, Introduction to Automata Theory, Language, and Computation, ISBN: 0-201-02988-X, (1979) AddisionWesley Publication Co.

[54] M. Fannes, B. Nachtergaele, and R. F. Werner, Ground States of $V S B$-Models on Cayley Trees, J. of Statist. Phys., 66, (1992) 939 - 973. 
[55] M. Fannes, B. Nachtergaele, and R. F. Werner, Finitely Correlated States on Quantum Spin Chains, Comm. Math. Phys., 144, no. 3, (1992) 443 - 490.

[56] M. Fannes, B. Nachtergaele, and R. F. Werner, Entropy Estimates for Finitely Correlated States, Ann. Inst. H. Poincare. Phys. Theor., 57, no 3, (1992) $259-277$.

[57] M. Fannes, B. Nachtergaele, and R. F. Werner, Finitely Correlated Pure States, J. of Funt. Anal., 120, no 2, (1994) 511 - 534.

[58] J. Renault, A Groupoid Approach to $C^{*}$-Algebras, Lect. Notes in Math., 793, ISBN: 3-540-09977-8, (1980) Springer.

[59] S. Sakai, $C^{*}$-Algebras and $W^{*}$-Algebras, MR number: MR0442701, (1971) Springer-Verlag.

Saint Ambrose Univ., Dep. of Math., 421 Ambrose Hall, 518 W. Locust St., Davenport, Iowa, 52803, U. S. A. / Univ. of Iowa, Dep. of Math, 25McLean Hall, Iowa City, IowA, 52242, U. S. A.

E-mail address: chowoo@sau.edu, Jorgense@math.uiowa.edu 